\title{
1 An amphipathic helix in Brl1 is required for membrane fusion 2 during nuclear pore complex biogenesis in S. cerevisiae
}

3 Annemarie Kralt $^{1} \uparrow$, Matthias Wojtynek $^{1,2} \dagger$, Jonas S. Fischer ${ }^{1} \dagger$, Arantxa Agote-Aran ${ }^{1}$, Roberta 4 Mancini $^{1}$, Elisa Dultz ${ }^{1}$, Elad Noor ${ }^{3}$, Federico Uliana ${ }^{1}$, Marianna Tatarek-Nossol ${ }^{4}$, Wolfram Antonin ${ }^{4}$, 5 Evgeny Onischenko ${ }^{5}$, Ohad Medalia ${ }^{2}$, Karsten Weis $^{1 *}$

$7 \quad{ }^{1}$ Institute of Biochemistry, Department of Biology, ETH Zurich, Zurich CH-8093, Switzerland

$8 \quad{ }^{2}$ Department of Biochemistry, University of Zurich, Zurich CH-8057, Switzerland

$9 \quad{ }^{3}$ Department of Plant and Environmental Sciences, Weizmann Institute of Science, Israel

$10{ }^{4}$ Institute of Biochemistry and Molecular Cell Biology, Medical School, RWTH Aachen University, 1152074 Aachen, Germany

$12{ }^{5}$ Department of Biological Sciences, University of Bergen, Bergen 5020, Norway

$13 \dagger$ These authors contributed equally to this work.

$14 *$ To whom correspondence should be addressed: karsten.weis@bc.biol.ethz.ch

\section{Abstract}

16 The nuclear pore complex (NPC) is the central portal for macromolecular exchange between the nucleus and cytoplasm. In all eukaryotes, NPCs assemble into an intact nuclear envelope (NE) during interphase, but the process of NPC biogenesis remains poorly characterized. Furthermore, little is known about how NPC assembly leads to the fusion of the outer and inner NE, and no factors have been identified that could trigger this event. Here we characterize the transmembrane protein Brll as an NPC assembly factor required for NE fusion in budding yeast. Brl1 preferentially associates with NPC assembly intermediates and its depletion halts NPC biogenesis, leading to NE herniations that contain inner and outer ring nucleoporins but lack the cytoplasmic export platform. Furthermore, we identify an essential amphipathic helix in the luminal domain of Brll that mediates interactions with lipid bilayers. Mutations in this amphipathic helix lead to NPC assembly defects, and cryo-ET analyses reveal multi-layered herniations of the inner nuclear membrane with NPC-like structures at the neck, indicating a failure in NE fusion. Taken together, our results identify a role for Brl1 in NPC assembly and suggest a function of its amphipathic helix in mediating the fusion of the inner and outer nuclear membranes. 


\section{Introduction}

Virtually all biological processes are carried out by multiprotein complexes, and their faithful assembly is therefore crucial for cellular function (Hartwell et al. 1999). The nuclear pore complex (NPC) is one of the largest cellular protein complexes, with a total mass of 60-120 MDa. In all eukaryotes, NPCs perforate the double lipid bilayer of the nuclear envelope (NE) and mediate macromolecular exchange between nucleus and cytoplasm (Wente and Rout 2010). NPCs are assembled from multiple copies of $\sim 30$ different proteins known as nucleoporins (NUPs), which amount to hundreds of proteins in the mature complex due to the NPC's eight-fold rotational symmetry (Fernandez-Martinez and Rout 2021; Lin and Hoelz 2019). NUPs are organized in well-defined sub-complexes (Figure 1A) where the membrane ring (MR), the central channel (CC) and the inner ring (IR) in the plane of the NE are sandwiched by two outer rings composed of Y-complexes. Asymmetrically attached to this scaffold are the cytoplasmic export platform (CP) and the nuclear basket (NB) (Figure 1A) (Fernandez-Martinez and Rout 2021; Lin and Hoelz 2019).

The architecture of the NPC has recently been elucidated in great detail (Akey et al. 2022; Bley et al. 2021; Huang et al. 2021, 2022; Li et al. 2021; Mosalaganti et al. 2021; Petrovic et al. 2021; Schuller et al. 2021; Tai et al. 2022; Zhu et al. 2022; Zimmerli et al. 2022). Yet far less is known about how this gigantic complex assembles and gets embedded into the NE. In metazoan cells, which undergo an open mitosis, two types of NPC assembly mechanisms have been described: mitotic reassembly of NPCs at the end of cell division and de novo formation of NPCs during interphase (Doucet, Talamas, and Hetzer 2010; Otsuka and Ellenberg 2018; Schooley, Vollmer, and Antonin 2012). Organisms that undergo closed mitosis, such as the budding yeast Saccharomyces cerevisiae, exclusively rely on interphase NPC assembly to create new NPCs (Winey et al. 1997). Here, NUP complexes punch a hole into the intact NE in order to create the protein-lined membrane tunnel that spans the NE. This requires a poorly understood fusion event between the inner (INM) and outer (ONM) nuclear membranes during which the integrity of the NE diffusion barrier is not compromised (Doucet and Hetzer 2010; Rothballer and Kutay 2013).

NPC assembly events are rare (e.g., in yeast 1-2 NPCs form per minute) (Winey et al. 1997) and capturing them in situ has been challenging. Therefore, NPC biogenesis has mainly been studied using genetic perturbations that inhibit its maturation. A shared phenotype of many NPC assembly mutants is the appearance of NE herniations, which likely correspond to halted NPC assembly intermediates (Thaller and Patrick Lusk 2018). The orientation of these herniations - always bulging out towards the cytoplasm - suggests an inside-out mechanism of NPC assembly, which is also supported by observations of interphase assembly states in human cells (Otsuka et al. 2016). To characterize the precise maturation order and assembly kinetics of native NPC biogenesis in budding yeast, we recently developed a mass spectrometry-based approach that we termed KARMA (Kinetic Analysis of 


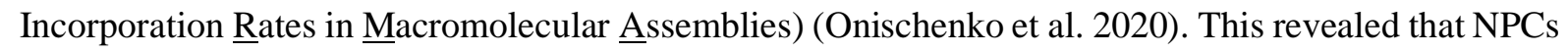
form by sequential assembly of nucleoporins starting with the central scaffold, followed by the outer cytoplasmic and nucleoplasmic parts and concluded by the late binding of Mlp1, consistent with an inside-out assembly mechanism (Onischenko et al. 2020).

To date, very few non-NPC proteins have been shown to participate in NPC assembly. This is in contrast to, e.g., ribosome biogenesis, where approximately 180 trans-acting assembly factors are known to interact during the maturation process. These are critical for ribosome-assembly but are not part of the final structure (Kressler, Hurt, and Bassler 2010; Strunk and Karbstein 2009). The few proteins suggested to promote interphase NPC assembly include the membrane-bending reticulons (Dawson et al. 2009), Torsin ATPases (Laudermilch et al. 2016; Rampello et al. 2020), the Ran GTPase and its regulators (Ryan, McCaffery, and Wente 2003), and, in budding yeast, a group of three small NE/ERlocated transmembrane proteins: Brl1, its paralogue Brr6 and Apq12 (De Bruyn Kops and Guthrie 2001; Hodge et al. 2010; Lone et al. 2015; Saitoh, Ogawa, and Nishimoto 2005; Scarcelli, Hodge, and Cole 2007; Zhang et al. 2018, 2021). Temperature-sensitive alleles of BRL1 and BRR6 or deletion of APQ12 show NE-herniations, an altered cellular membrane composition, synthetic interactions with lipid biosynthesis pathways and sensitivity to drugs influencing membrane fluidity (Hodge et al. 2010; Lone et al. 2015; Scarcelli et al. 2007; Zhang et al. 2021). Brl1, Brr6 and Apq12 can be coimmunoprecipitated, which suggests they form a complex (Lone et al. 2015), and they have been found to physically interact with NUPs (Zhang et al. 2018). Interestingly, overexpression of Brl1 but not Brr6 can bypass the function of Nup116 and Gle2 in NPC assembly (Liu et al. 2015; Zhang et al. 2018), suggesting that Brl1 and Brr6 act differently during NPC maturation.

Here, we take advantage of our KARMA method (Onischenko et al. 2020) to identify NPC biogenesis factors. We show that Brl1 transiently binds to immature NPCs and that depletion of Brl1 impairs NPC assembly, resulting in NE herniations that contain the central scaffold NUPs but lack the cytoplasmic export platform (Nup82, Nup159). We further identify an essential luminal amphipathic helix (AH) in Brl1 that interacts with membranes and, when mutated, leads to the formation of large, multi-layered NE herniations containing immature NPCs that we structurally characterize by cryo-electron tomography. Our results identify Brl1 as an essential NPC assembly factor and suggest that Brl1 mediates the fusion step between the inner and outer nuclear membranes during interphase NPC biogenesis via its $\mathrm{AH}$. 
96

97

98

99

100

101

102

103

104

105

106

107

108

109

110

111

112

113

114

115

116

117

118

119

120

121

122

123

124

\section{Results}

\section{Brl1 binds to assembling nuclear pore complexes}

Relying on a large KARMA dataset that contains kinetic interaction profiles for 10 different NUP baits, we recently demonstrated that yeast NPCs assemble sequentially, starting with the symmetrical core NUPs (early tier), followed by the majority of asymmetric NUPs (intermediate tier), and concluded by the assembly of two nuclear basket NUPs Mlp1 and Mlp2 (late tier) (Figure 1A-1B) (Onischenko et al. 2020). This analysis also identified a large number of non-NUP proteins that interact with the baits. We sought to exploit our dataset to uncover potential NPC assembly factors. Since such factors are expected to selectively bind to the NPC during its biogenesis but are not part of the mature structure, they should be enriched in early tier NUP pulldowns versus late tier ones (Figure 1B). Interestingly, out of 1'500 co-purified non-NUP proteins, Brl1 displayed the second highest enrichment score (Figure S1A), decreasing in abundance approximately five-fold from early to late tier baits (Figure 1C). Only Her1, a protein with unknown biological function, had a higher early-to-late enrichment ratio. Brll has previously been implicated in NPC biogenesis (Lone et al. 2015; Zhang et al. 2018), and to confirm its binding preference for early assembling NUPs, we performed the reciprocal affinity pulldowns with endogenously tagged Brl1. In full agreement, early tier NUPs were enriched over the ones from intermediate and late assembly tiers (Figure S1B).

3 Brll's preference for 'young' NPCs was validated by live-cell imaging using the recombinationinduced tag exchange (RITE) approach (Verzijlbergen et al. 2010). We genetically tagged Nup170, which binds early during NPC biogenesis, with a RITE construct. This allowed us to specifically mark either old or newly synthesized Nup170 by removing or introducing a yEGFP-tag through inducible genetic recombination (Figure 1D). Since Nup170 binds early during NPC biogenesis, it can be assumed that some of the foci formed by newly synthesized Nup170-yEGFP represent NPC assembly intermediates. As a measure of Brl1 association with young and old NPCs, we monitored co-localization between Brl1-mCherry and either new or old Nup170-yEGFP using cross-correlation of the NE fluorescence signals as the readout. As evidenced by a lower cross-correlation score and in agreement with our KARMA data, Brl1 co-localized well with young but not with old NPCs (Figure 1E-F). Together, these results indicate that Brl1 preferentially binds to young or immature NPCs, which is consistent with a function of Brl1 during NPC biogenesis. 
A

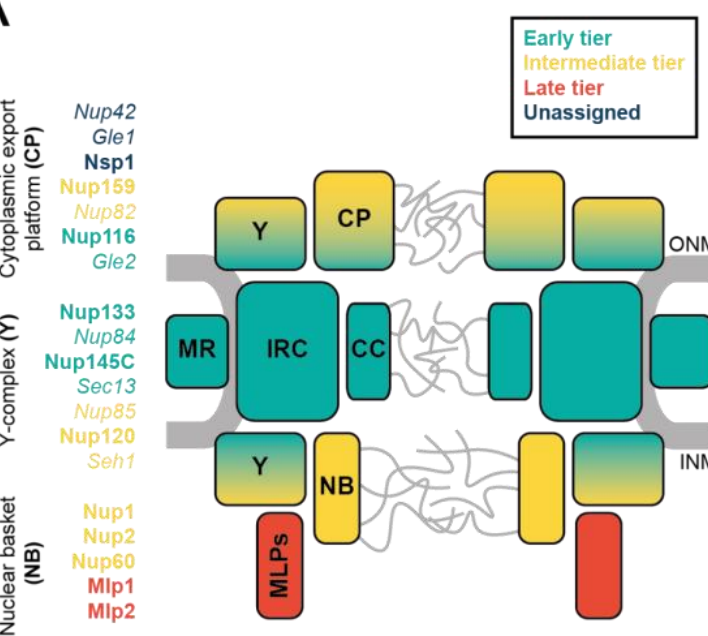

$D$

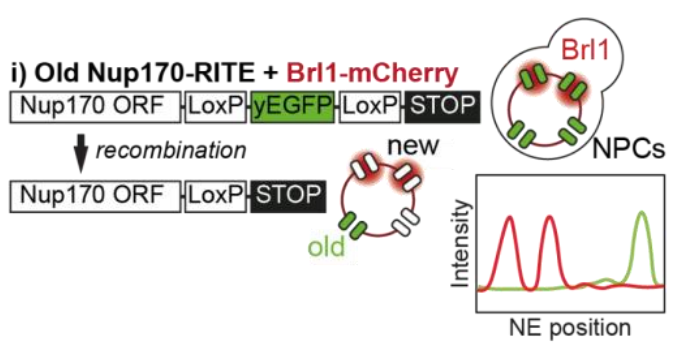

E
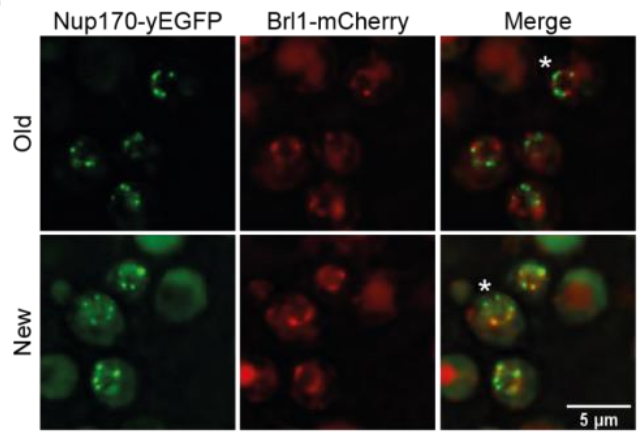

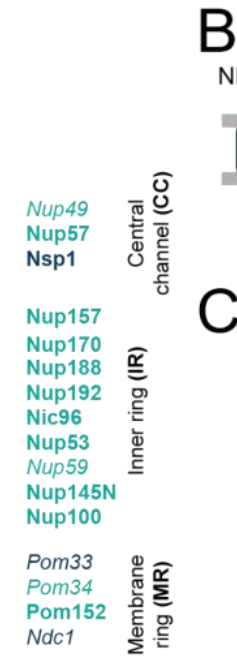

B

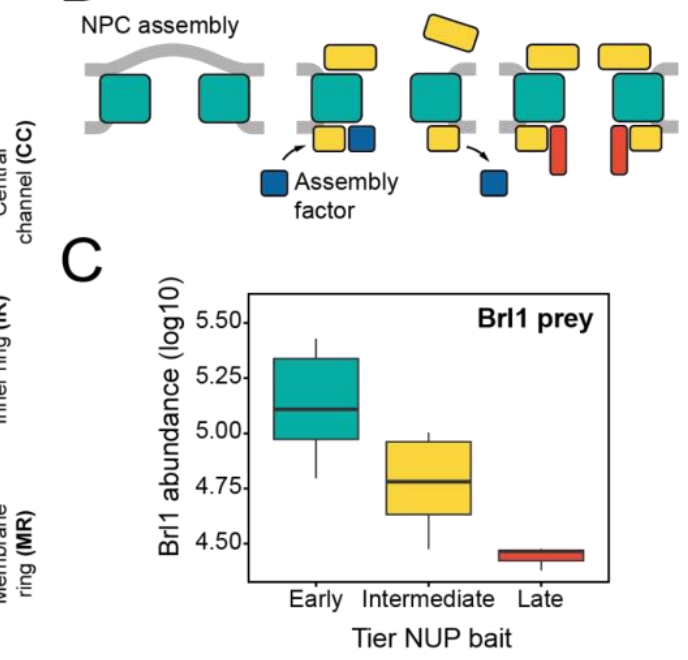

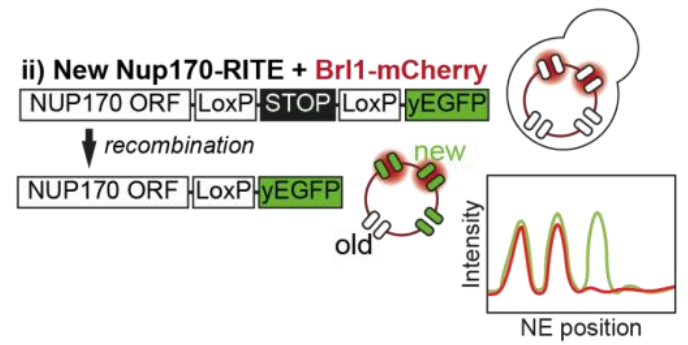

F
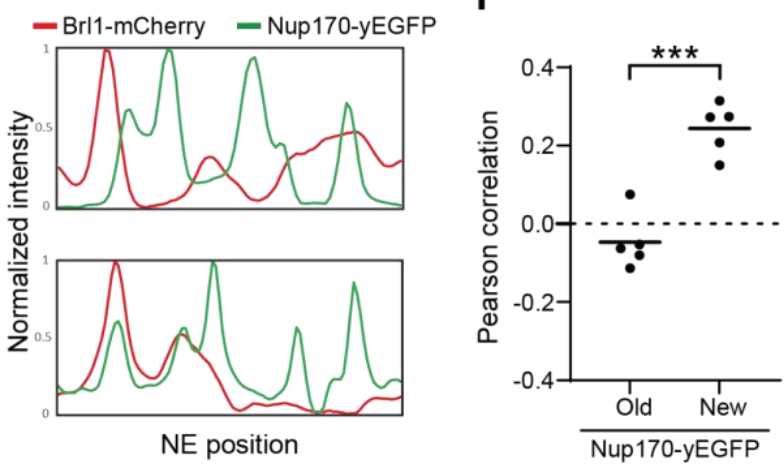

Figure 1: Brl1 preferentially binds young nuclear pore complexes. A) Scheme of the nuclear pore complex architecture. The colors indicate the assembly order as found in Onischenko et al. (Onischenko et al. 2020). NUPs that were reproducibly identified in Brl1 affinity purifications are shown in bold. B) Schematic illustrating the transient binding of an NPC assembly factor during NPC assembly. C) Enrichment of Brl1 in affinity pulldowns from Onischenko et al. (Onischenko et al. 2020) using baits from the different assembly tiers. Early and intermediate tiers contain four different baits each; the late tier is represented by Mlp1 with three biological replicates for each bait. D) Schematic representation of the RITE strategy to visualize Brl1-mCherry colocalization with old or new NPCs marked by Nup170-yEGFP and the expected NE fluorescence intensity profiles. E) Representative co-localization images of Brl1-mCherry with old or new Nup170-yEGFP marked NPCs using the RITE strategy described in 1D. Cells were imaged $\sim 30 \mathrm{~min}$ or $\sim 5 \mathrm{~h}$ after recombination induction, respectively. Fluorescence intensity profiles along the NE are displayed for cells denoted with an asterisk (*). F) Pearson correlation between Nup170-yEGFP and Brl1-mCherry fluorescence intensity profiles along the NE in 
1E. Individual points reflect the average of a biological replicate with a minimum of 28 analyzed NE contours per condition. Two tailed Student's t-test $(n=5$, $\mathrm{p}$ value $=0.00015)$.

Taking advantage of our KARMA workflow, we next set out to determine more precisely the stage during which Brl1 acts in NPC biogenesis. In KARMA, newly synthesized proteins are pulse labeled by heavy-isotope amino acids followed by the pulldown of the NPC via an endogenously tagged affinity bait at several post-labeling time points (Figure 2A) (Onischenko et al. 2020). The extent of metabolic labeling of any co-isolated protein is indicative of its average age in the affinity pulldown (AP) fraction (Figure 2A). Therefore, the 'young' structural intermediates that are bound by a bona fide assembly factor during biogenesis should display a higher metabolic labeling rate in APs compared to the labeling of bulk cellular proteins. By contrast, structural components that join after the assembly factor has left the NPC assembly site are not expected to show this effect, even if the assembly factor does not dissociate completely (Figure 2A). Nuclear transport receptors (NTRs) that bind the NPC highly transiently serve as a reference for bulk cellular protein labeling to discriminate between young and old proteins.

In KARMA assays with endogenously tagged Brl1, we were able to detect most NUPs (Figure 1A) with highly reproducible labeling readouts between biological replicates (Figure S1C, S1D). Strikingly, the NUP labeling rates observed with Brl1 as bait were overall significantly higher compared to the ones in KARMA assays with NUP baits (Onischenko et al. 2020) (Figure 2B). On top, we observed that in Brl1 pulldowns, early tier NUPs were labeled outstandingly fast, exceeding NUPs from the intermediate or late tiers and even the NTRs - our reference of the bulk cellular proteins (Figure 2B, 2D, S1D). In line with this, our quantitative analysis of NUP metabolic labeling rates using a previously developed kinetic state model (KSM) (Onischenko et al. 2020), revealed that early tier NUPs become inaccessible to the Brl1 bait in mature NPCs (Figure 2C) (Supplementary Results: "Kinetic state modeling"), likely as a result of the dissociation of Brl1 at later stages of NPC assembly (Figure 1B). Although most NUPs from late and intermediate tiers were still detected in the KARMA assays, they did not display elevated labeling rates and even showed significant labeling delays as in the case of Mlp1, Nup159 and Nsp1 (Figure 2D). Altogether these results show that Brl1 preferentially binds NPC assembly intermediates that are composed of the central scaffold (early tier) but lack the peripheral nucleoplasmic and cytoplasmic structures (intermediate and late tier) (Figure 2E). Of note, the labeling differences we observed cannot be explained by variations in NUP turnover as evidenced by the analysis of NUP labeling rates in the source cell lysates (Supplemental results: "Analysis of protein labeling in source lysate"). Moreover, it is likely that the observed contrast in the labeling rates is significantly underestimated due to intermixing of Brl1-purified NUP species during the AP procedure (Supplemental results "Lysis intermixing assays"). 
A
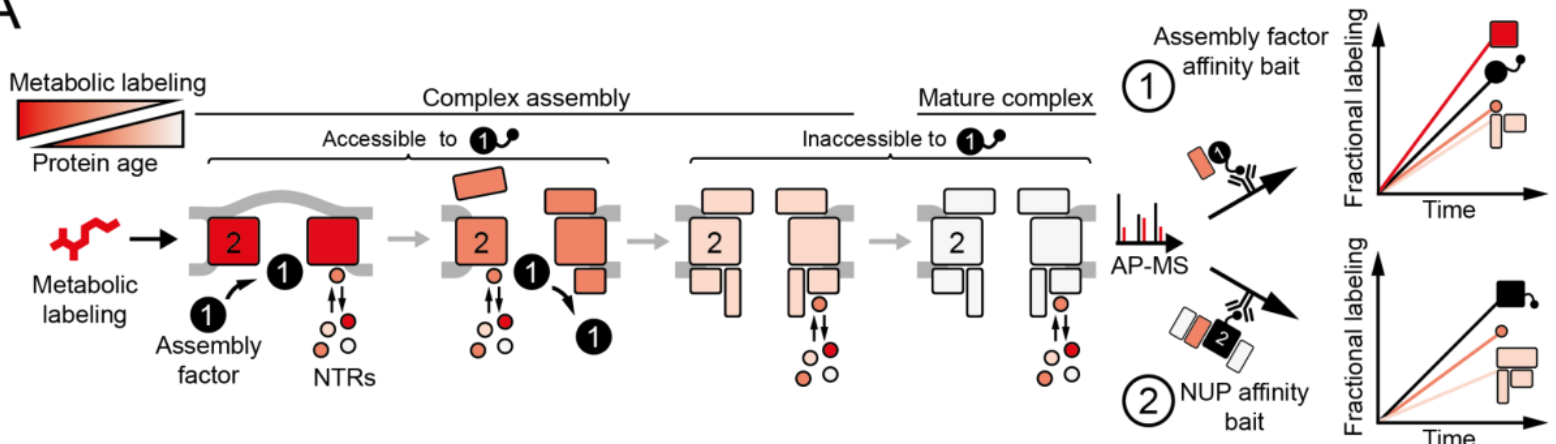

B

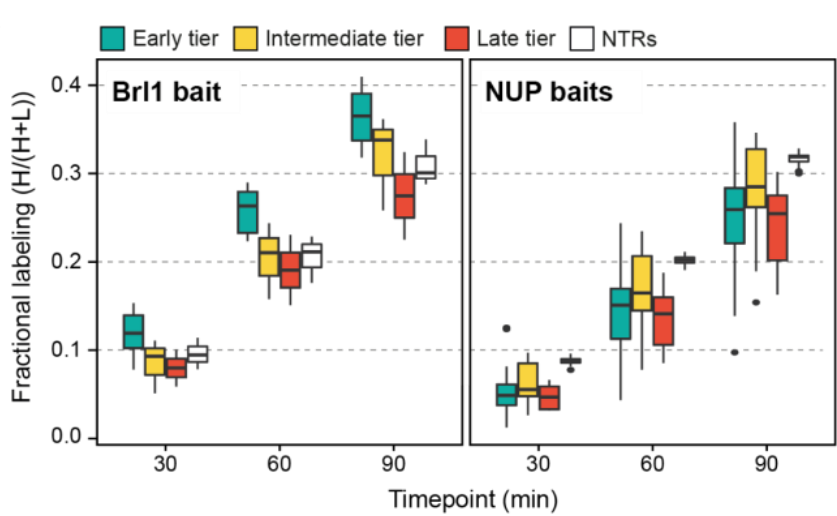

C

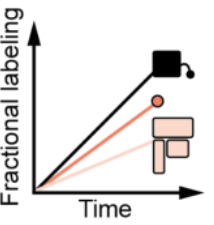

$\mathrm{D}$

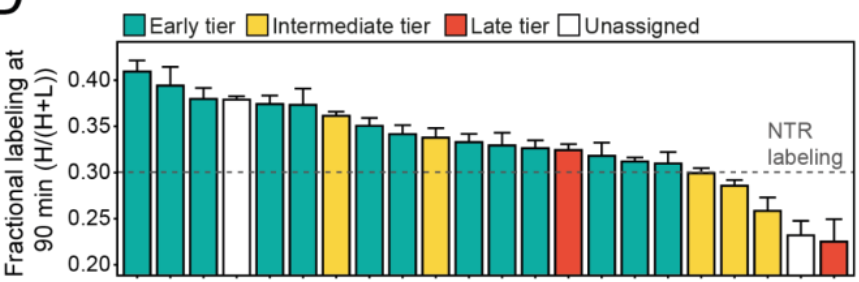

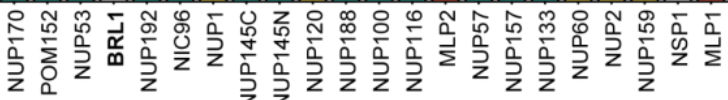

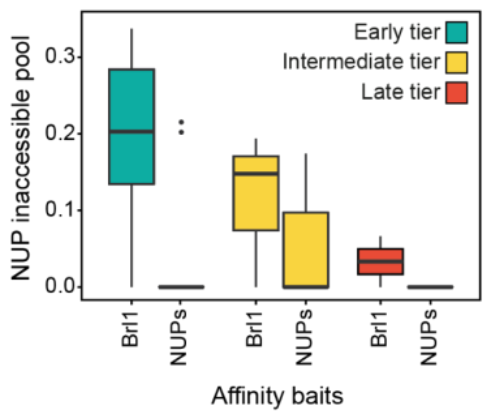

$E$

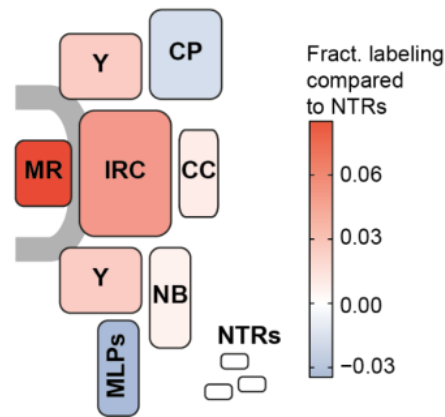

Figure 2: Mapping Brl1 association with NPC assembly intermediates using KARMA. A) Principles of

KARMA: Newly synthesized proteins are pulse-labeled followed by the affinity purification of the NUP complexes through a tagged NPC-binding protein. The extent of metabolic labeling is then quantified by mass spectrometry and corresponds to the average protein age in the affinity-purified fraction. An assembly factor selectively binds young NPCs, thus leading to high metabolic labeling rates for NUPs present in the intermediates (1). This is not the case for proteins that join after the assembly factor completely or partially dissociates or when the process is probed with a NUP bait (2). B) Comparison of the labeling rates for NUPs and NTRs in KARMA assays with Brll bait (left, this study) and with ten different NUP baits (right, (Onischenko et al. 2020)). Median of three biological replicates. C) Inaccessible pool of NUPs in KARMA assays with Brl1 compared to NUP baits (Onischenko et al. 2020), evaluated using a three-state KSM (Onischenko et al. 2020).D) Barplot depicting the extent of metabolic labeling for different NUPs in KARMA assays with Brl1 bait after 90 min. The dotted line indicates the median NTR labeling. Median \pm SD of three biological replicates. E) Fractional labeling values from 2D averaged for NPC sub-complexes and offset by NTR labeling projected onto the NPC scheme. 
A

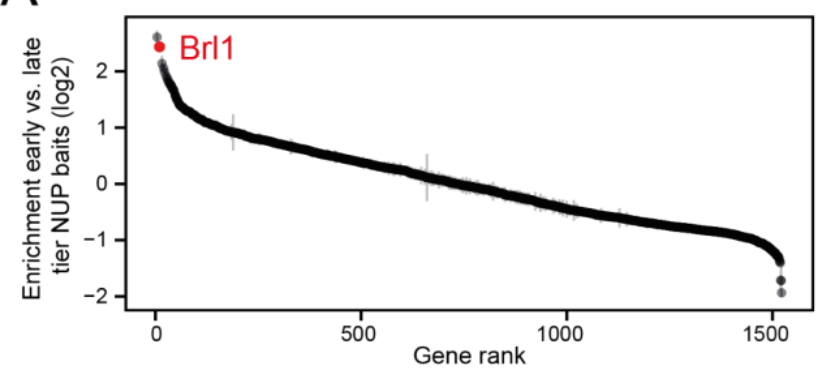

B

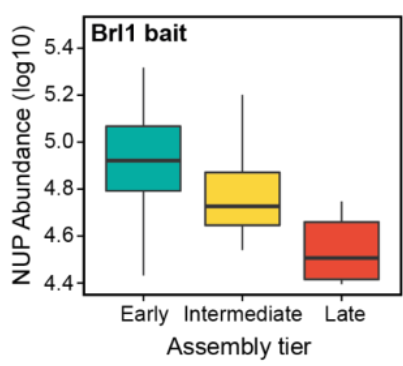

C

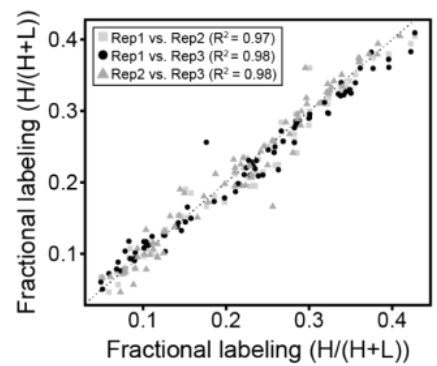

D

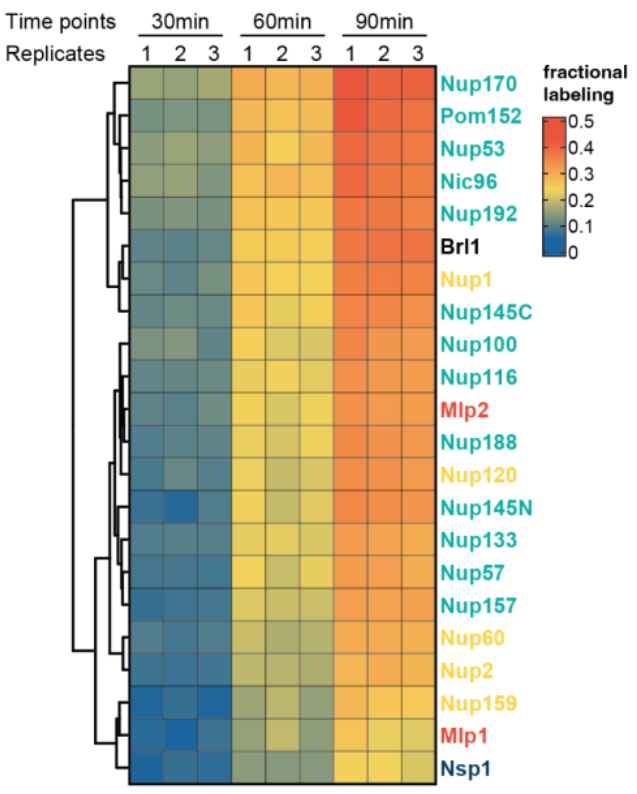

190

Figure S1: Proteomic characterization of Brl1 NPC interactions. A) All 1'500 proteins co-purified in affinity pulldowns with ten different NUP baits (Onischenko et al. 2020) were ranked by their fold enrichment difference between early and late tier baits. Mean \pm SEM of three biological replicates. B) Log10 abundance of NUPs belonging to the different assembly tiers in Brl1 APs. Values for three biological replicates. C) Reproducibility of the fractional labeling in KARMA assays with Brl1. Individual points correspond to the fractional labeling of a protein. D) Heatmap showing the fractional labeling of NUPs in KARMA assays with Brl1 bait.

\section{Depletion of Brl1 interferes with NPC maturation}

Having established that Brl1 interacts with immature NPCs, we wanted to elucidate how the absence of Brl1 affects NPC assembly. Since Brl1 is encoded by an essential gene, we used the auxin-inducible degron (AID) system, which allows for the acute depletion of proteins (Figure S2A) (Nishimura et al. 2009). Upon addition of auxin, $~ 65 \%$ of Brl1 was rapidly degraded within 15 minutes (Figure S2B), leading to a reduction in growth rate (Figure S2C). To characterize whether Brl1 degradation affected the NPC ultrastructure, we treated cells for 4-4.5 hours with auxin and then subjected them to cryofocused ion beam (FIB) milling and cryo-electron tomography (cryo-ET). As expected, we found mature NPCs (Figure 3A ii white arrow and Movie S1) in the NE of auxin-treated cells, but also detected small electron-dense INM evaginations (Figure 3A iii and Movie S2) along the NE. Additionally, we observed that Brl1-depleted cells have electron-dense NE herniations (Figure 3A black arrows and Movies S1 and S2) similar to the ones commonly observed in NPC assembly mutants (Thaller and Patrick Lusk 2018) and previously also seen for Brl1/Brr6 double-depleted cells (Zhang et al. 2018). In our control strain lacking the auxin receptor OsTir1, no herniations could be detected after auxin 
212 treatment (Figure 3B, Movies S3 and S4). However, we infrequently observed INM evaginations

213 (Figure 3B, Movie S3), indicating that these could represent regular NPC intermediates.

214 Interestingly, the herniations that we observed upon Brl1 degradation were often clustered and enclosed

215 by a continuous ONM (Figure 3A ii and iv, Movies S1 and S2). Closer inspection revealed densities

216 likely corresponding to the inner ring (IR, Figure 1A) at the apex of the INM (Figure S2D ii).

217 Subtomogram averaging and single subtomograms of the NE herniations also indicate the presence of

218 a nucleoplasmic density, likely corresponding to the nucleoplasmic Y-complex ring as previously

219 reported by Allegretti and coworkers (Figure S2D ii) (Allegretti et al. 2020). While the subtomogram

220 averaging of INM evaginations did not reveal distinct densities likely because of their high

221 heterogeneity and the limited number of analyzed subtomograms, the average of mature NPCs extracted

222 from the same dataset displayed a similar architecture as previously reported in higher resolution

223 subtomogram averages (Akey et al. 2022; Allegretti et al. 2020) (Figure S2D ii and iii, S2E).

224 Occasionally we also observed luminal densities at the herniations, likely corresponding to the Pom152

225 luminal ring (Akey et al. 2022; Upla et al. 2017; Zimmerli et al. 2022) (Figure S2F). This is in line with

226 our KARMA data, suggesting that Pom152 is already present in assembling NPCs prior to Brl1

227 recruitment (Figure 2D-2E and S1D).

228 To further characterize the composition of the NPC intermediates in Brl1-depleted cells, we investigated

229 the localization of yEGFP-tagged Nups after auxin addition (Figure 3C-3D). Consistent with our EM

230 data, the inner ring complex NUPs (Nup170 and Nup192), the Y-complex members (Nup133 and

231 Nup85) and linker NUPs (Nup100 and Nup116) retained a prominent NE localization, while the

232 cytoplasmic export platform NUPs (Nup82 and Nup159) were mislocalized in bright foci. Interestingly,

233 the nuclear basket NUPs (Nup60 and Mlp1) also readily localized at the nuclear envelope. We thus

234 conclude that NPC structures that accumulate upon Brl1 depletion contain the central scaffold and the

235 nuclear basket structure but lack the cytoplasmic face of the NPC (Figure 4D right).

236 To exclude that mature NPCs are affected by the depletion of Brl1, we monitored NUPs synthesized

237 before and after Brl1 depletion separately using RITE (Figure 3E) (Verzijlbergen et al. 2010). New

238 Nup188, Nup133 and Mlp1 still localize to the NE homogeneously, whereas new Nup82 forms bright

239 foci either in the cytoplasm or NE (Figure 3F). By contrast, the localization of old proteins was not

240 affected for any tested NUP. Together, our results reveal that removal of Brl1 triggers the formation of

241 NE herniation as a consequence of halted NPC assembly, whereas previously assembled NPCs are not

242 affected by the lack of Brl1. 
A

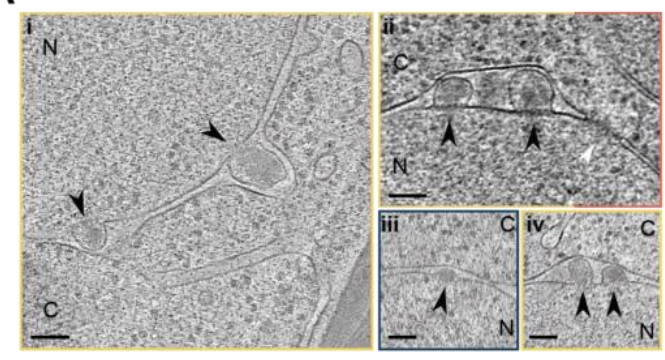

C
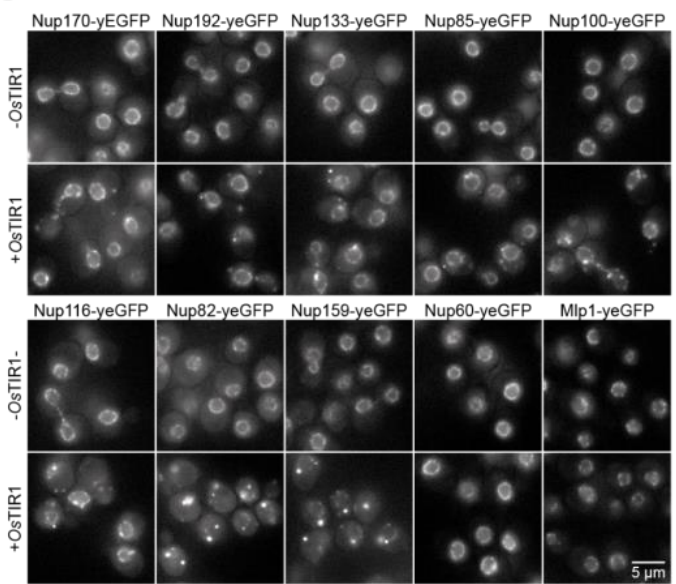

B

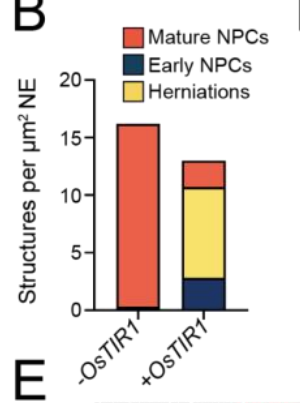

E
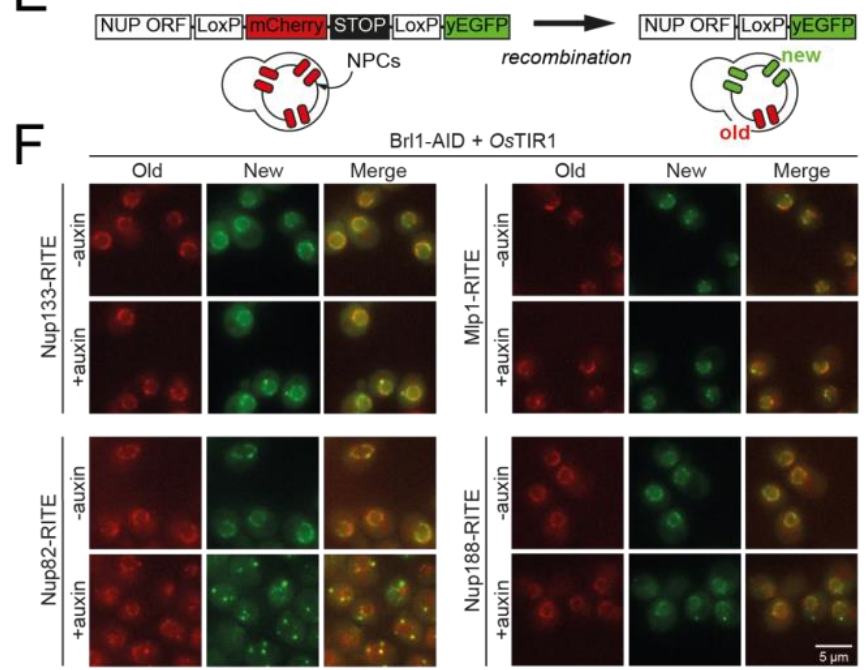

Figure 3: Brl1 degradation interferes with NPC assembly. A) Tomographic slices of FIB-milled, 4-4.5 h auxintreated Brl1-AID cells showing the structures quantified in 3B. Image frames colored according to the color code used in B). Scale bar $100 \mathrm{~nm}$, black arrows: herniations, white arrow: NPC, N: Nucleus, C: Cytoplasm; slice thickness i and iii: $1.4 \mathrm{~nm}$, ii and iv: $2.8 \mathrm{~nm}$. Panels i and ii are cropped from tomographic slices from the tomograms in movies S1 and S2 B) Quantification of 27 tomograms $\left(8.5 \mu \mathrm{m}^{2} \mathrm{NE}\right)$ and $51\left(16.7 \mu \mathrm{m}^{2} \mathrm{NE}\right)$ for OsTir1 and +OsTir1 respectively. C) Example fluorescent micrographs of yEGFP-tagged NUPs in 4-4.5 h auxin treated Brl1-AID +/- OsTIR1 cells. D) Normalized fluorescence intensity signal in the nuclear envelope in +/OsTIR1 Brl1-AID cells treated with $500 \mu \mathrm{M}$ auxin for 4-4.5 h. Mean \pm SEM of a minimum of two biological replicates. E) Recombination-induced tag exchange (RITE) method is combined with a CRE-EBD recombinase to conditionally switch fluorescence tags upon $\beta$-estradiol addition. F) NUP RITE fusion protein localization in the Brl1-AID background $3 \mathrm{~h}$ after treating cells with auxin (+auxin) or ethanol (-auxin). Recombination was induced 30 min prior to auxin addition. 
bioRxiv preprint doi: https://doi.org/10.1101/2022.03.04.483005; this version posted March 4, 2022. The copyright holder for this preprint (which was not certified by peer review) is the author/funder, who has granted bioRxiv a license to display the preprint in perpetuity. It is made available under aCC-BY-NC-ND 4.0 International license.
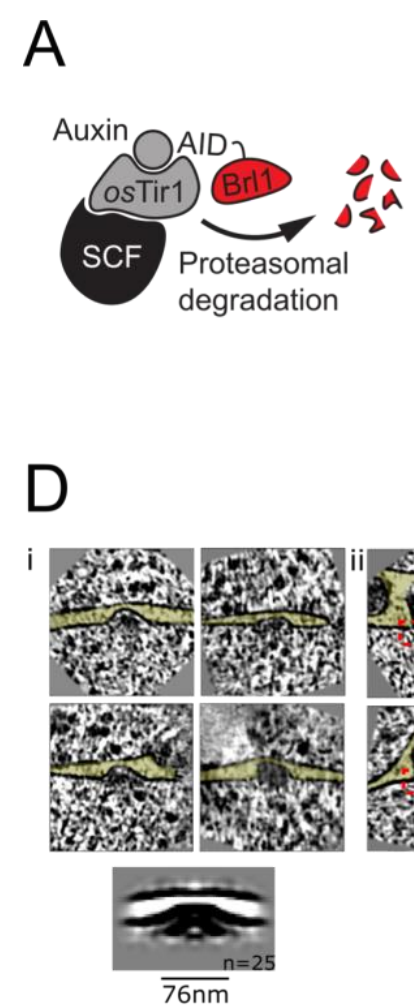

$\mathrm{F}$
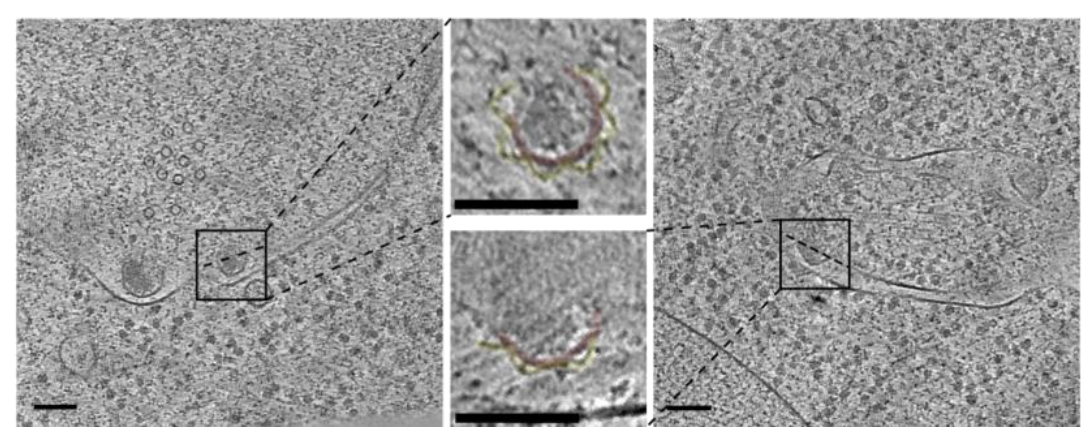
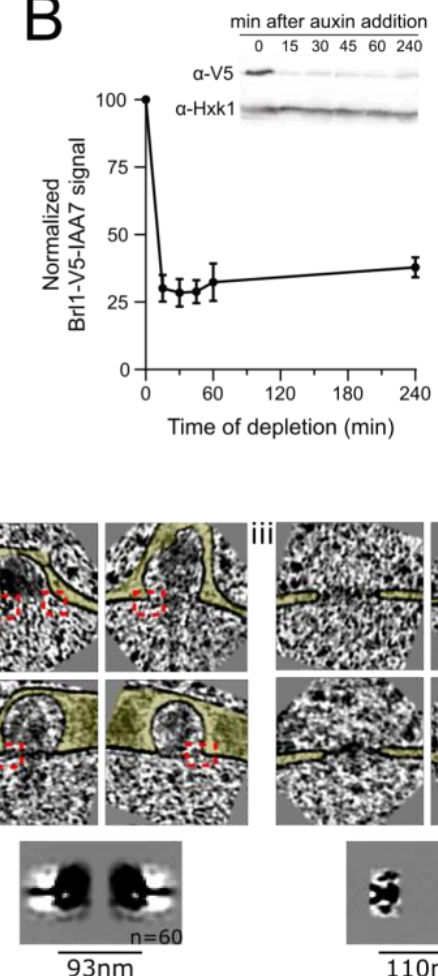

258

259

260

261

262

263

264

265

266

267

268

269

270

271

Figure S2: Characterization and subtomogram analysis of Brl1 depletion. A) Auxin-inducible degradation of Brl1 (Nishimura et al. 2009). Interaction between degron-tagged Brl1 and the E3 ubiquitin ligase SCF is mediated by the auxin binding receptor OsTir1. B) Depletion of Brl1-AID monitored by Western blotting. Brl1V5-IAA7 was detected with an anti-V5 antibody, anti-Hexokinase served as loading control. Mean \pm SEM of three biological replicates. C) Growth rate of Brl1-AID $\pm O s$ Tirl cells incubated with $500 \mu \mathrm{M}$ auxin or an equivalent amount of ethanol (-auxin). D) Subtomograms and subtomogram averages of NPCs and NPC-like structures in Brl1-depleted conditions; (i) INM-evaginations, (ii) NE-herniations, (iii) mature NPCs. Diameter and number of particles are indicated. Cytoplasm is pointing up in all images. Box size of single herniations/NPCs is $270 \mathrm{~nm}$. E) Fourier shell correlation curves for the subtomogram averages in figure S2D and figure $7 \mathrm{C}$. FSC 0.5 indicated as dotted line F) Tomographic slices of FIB-milled 4-4.5h auxin treated Brl1-AID cells; Slices through herniations show a luminal ring around the herniation, highlighted in yellow, NPC-membrane in red; the rotationaxis is indicated by a dashed line; scale bars: $100 \mathrm{~nm}$; slice thickness: $1.4 \mathrm{~nm}$. 
272 To systematically explore the composition of the NPC assembly intermediates that accumulate in the 273 absence of Brl1, we once more employed metabolic labeling coupled to affinity purification mass 274 spectrometry. We used Nup170 as an affinity bait since it binds early during NPC maturation 275 (Onischenko et al. 2020) enabling us to purify both mature NPCs and intermediate structures upon Brl1 276 depletion (Figure 4A). To this end, we pulse-labeled newly synthesized proteins in parallel with the 277 induction of Brl1 degradation, and subsequently quantified the metabolic labeling for all co-purified 278 proteins. For NUPs that are able to assemble into intermediates in the absence of Brl1, we expect to 279 find a mixture of unlabeled (old) and labeled (new) proteins in Nup170 APs. However, for NUPs 280 dependent on Brl1 for their assembly, only pre-assembled, old proteins will be captured. Thus, proteins 281 dependent on Brl1 for their incorporation are expected to have slower labeling rates (Figure 4A).

282 In Brl1-depleted cells, the metabolic labeling of NUPs was generally slower than for the bulk of co283 purified proteins. Such a delay was not observed in control cells implying that the NPC maturation 284 process is affected when Brl1 is depleted (Figure 4B). Importantly, the labeling delay was not identical 285 for all NUPs (Figure 4C). While most membrane ring, nuclear basket, and inner ring complex NUPs 286 were labeled comparable to the dynamic NTRs, the cytoplasmic export platform NUPs and Mlp1 287 incorporated labeling substantially slower (Figure 4D left). This is in agreement with the densities 288 observed by cryo-ET and corroborates that the observed herniations are indeed incomplete NPC 289 assembly intermediates that have not yet acquired the cytoplasmic structure and that Mlp1 is recruited 290 very late to the NPC. Of note, the differences in NUP labeling observed upon Brl1 depletion with 291 Nup170 correlate well with the labeling rates in KARMA assays with Brl1 bait (Figure S3A). This 292 indicates that most NUPs which assemble after the Brl1-dependent assembly step (slow labeling in 293 KARMA assays with the Brl1 bait) can no longer incorporate into the NPC once Brl1 is degraded (slow 294 labeling in KARMA assays when Brl1 is depleted).

295 Of note, the metabolic labeling of the bulk of co-purified proteins was also overall delayed upon Brl1 296 depletion (Figure 4B). This is consistent with the decreased growth rate that can be observed in these 297 conditions (Figure S2C). Interestingly, the analysis of NUP exchange rates during the AP procedure 298 using cell lysate intermixing assays showed a significantly higher degree of exchange in the Nup170 299 APs when Brl1 was depleted (Figure S3B-S3D). This might suggest that the immature pores that 300 accumulate in the absence of Brl1 are less stable than fully assembled NPCs. Importantly, such dynamic 301 exchange leads to the intermixing of labeled and unlabeled constituents of the NPC and thus the labeling 302 delays that we observe in the Nup170 APs are likely underestimations. 

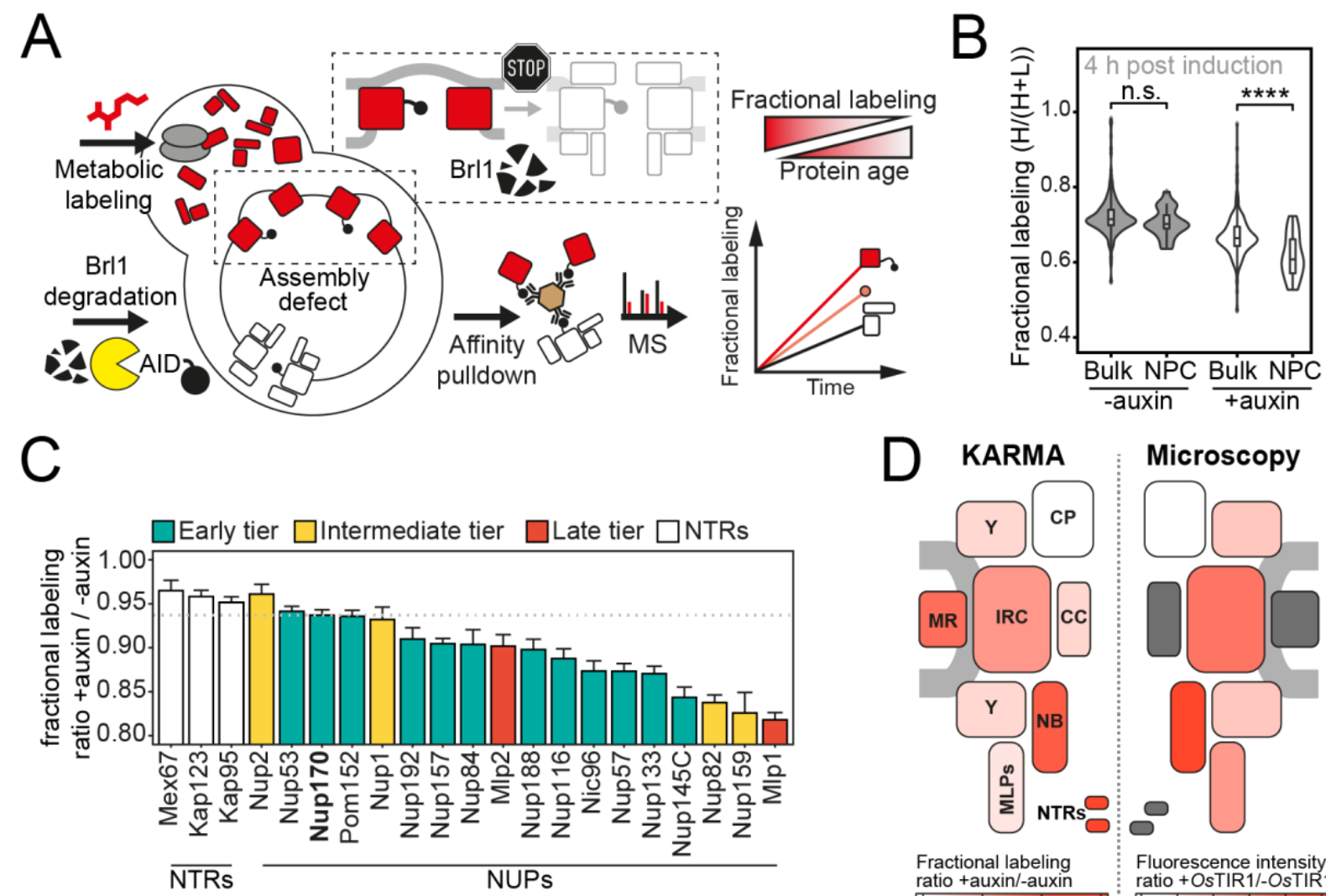

Figure 4: Proteomic characterization of NPC assembly intermediates induced by Brl1 depletion. A)

Depiction of the metabolic labeling assays to examine NPC assembly effects that occur upon Brl1 degradation.

Newly synthesized proteins are pulse-labeled simultaneously with the auxin-induced depletion of Brll. Mature NPCs and assembly intermediates are purified via affinity tagged Nup170. Newly made NUPs that depend on Brl1 for their incorporation cannot be purified with Nup170, thus diminishing the extent of their metabolic labeling in Nup170 AP after Brl1 depletion. B) Fractional labeling of bulk proteins compared to NUPs in KARMA assays with affinity tagged Nup170 in Brl1-AID cells treated with auxin (+auxin) or ethanol (-auxin) for $4 \mathrm{~h}$. Data points correspond to the median values in three biological replicates. Two tailed Student's t-test ( $\mathrm{p}$ value: n.s. $>0.05$ and $* * * *<0.0001$ ). C) Fractional labeling ratio of NUPs (bars) and bulk proteins (dotted line) in Nup170 APs from Brl1-AID cells treated with auxin (+auxin) or ethanol (-auxin). Mean \pm SEM of three biological replicates and three time points $(4,4.5$ and $5 \mathrm{~h}$ post treatment, $\mathrm{n}=9)$. Mlp1 and Mlp2 are missing in one replicate of the 4.5 time point $(n=8)$. D) Left: fractional labeling ratios from 4 C averaged per sub-complex and projected onto the NPC schematic. Right: Nuclear envelope fluorescence intensity signal ratio from Figure 3D averaged for NPC subcomplexes and projected onto the NPC schematic 
A

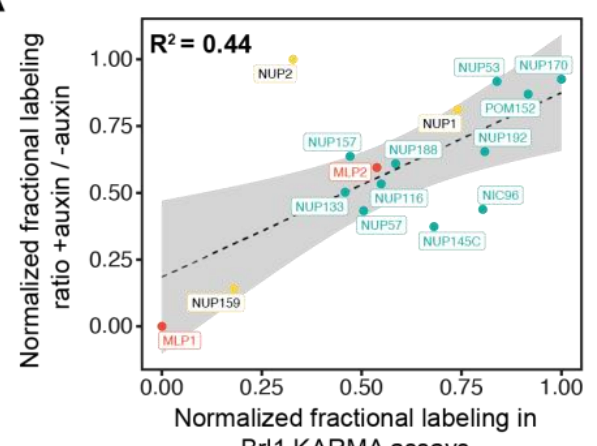

C

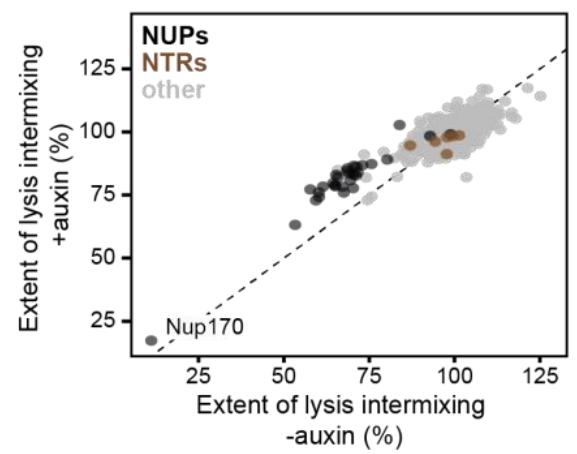

B

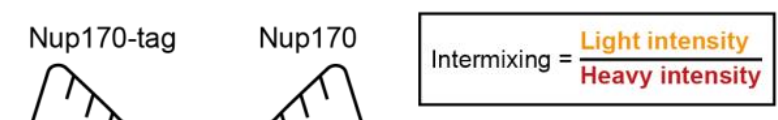

Metabolic labeling due to intermixing

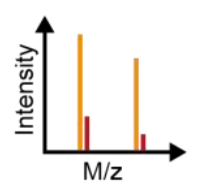

D

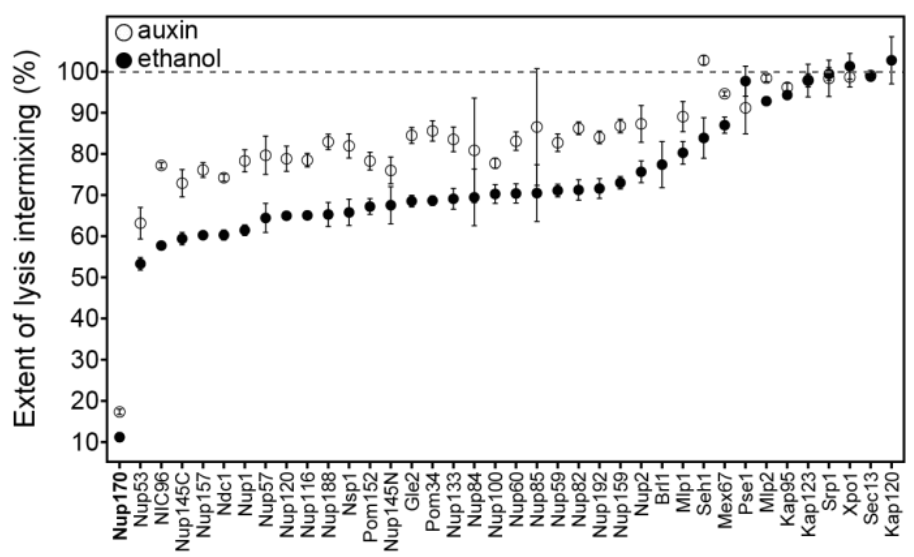

Figure S3: Exchange rates of NPC assembly intermediates in Brl1-depleted cells. A) Correlation between NUP fractional labeling observed in Brl1 KARMA assays with the fractional labeling ratios in Nup170 AP from Brl1-AID cells with auxin or ethanol treatment. B) Lysate intermixing assay to test the extent of dynamic exchange during the AP procedure. Brl1-AID cells are either treated with auxin or ethanol for $5 \mathrm{~h}$, then equal fractions of cell culture expressing tagged Nup170, grown in light lysine medium and wild type culture grown in metabolic labeled medium were subjected to the AP procedure. C) Intermixing of NUPs (black) and NTRs (brown) along with other co-purified proteins (gray) with auxin or ethanol treatment. Values are normalized to the mean intermixing of all co-purified non-NUP proteins in a sample (=100\%). D) Intermixing extent for NUPs and NTRs in Nup170 APs in Brl1-AID cells treated with auxin (black) or ethanol (white). Normalized as in 3C. Median \pm SD of three biological replicates. 
Brl1 contains an essential luminal amphipathic helix

So far, our analyses showed that Brl1 is an NPC assembly factor: it predominantly interacts with immature NPCs preceding incorporation of the cytoplasmic export platform and its depletion leads to the formation of NE herniations with a continuous ONM, suggesting that Brl1 may act prior to INMONM fusion during NPC maturation. We therefore wanted to mechanistically understand how Brl1 promotes NPC biogenesis. Brl1 is composed of a long unstructured N-terminus and two transmembrane domains linked by a luminal domain, which contains four cysteines that form two disulfide bridges (Figure 5A, 5C and S4D - G) (Zhang et al. 2018). Such a structural organization was also predicted by AlphaFold (Figure 5A and Figure S4) (Jumper et al. 2021). The structured part of Brl1 containing the transmembrane and luminal region were predicted with high confidence scores and agree well with previous experimental findings (Saitoh et al. 2005; Zhang et al. 2018). The N- and C-terminus on the other hand had poor prediction scores, as expected for natively disordered regions (Figure S4A-C). Closer inspection of the predicted Brl1 structure revealed an amphipathic helix (AH) just upstream of the second transmembrane domain (Figure 5A-C), that was also suggested by the amphipathic helix prediction algorithm HeliQuest (Gautier et al. 2008) (Figure 5C).

Amphipathic helices are short motifs capable of binding lipid bilayers and they have been implicated in bending membranes by inserting into one leaflet of a bilayer, generating a convex curvature (Ford et al. 2002; Wang et al. 2016). Interestingly, AHs are structural features of many membrane-binding NUPs (Hamed and Antonin 2021) and likely target NUPs to the NPC by curvature sensing (Floch et al. 2015). The amphipathic helix in Brl1 (ahBrl1) is highly conserved between organisms with closed mitosis (Figure 5C), suggesting that it could play a critical role in NPC biogenesis, for example by mediating the INM-ONM fusion. Indeed, in tetrad dissections of heterozygous yeast strains carrying a mutant allele of $B R L 1$ either lacking the $\mathrm{AH}(\operatorname{brll} \triangle a h)$ or disrupting the $\mathrm{AH}(\operatorname{brll}(\mathrm{I} 395 \mathrm{D}))$, only the two spores that carried the wild-type allele were viable (Figure 5D). This shows that ahBrll is essential for the function of Brl1 and cell viability.

We hypothesized that ahBrl1 might contribute to the INM-ONM fusion step in NPC biogenesis through interaction with membranes. We therefore tested the membrane binding capacity of ahBrl1 in vitro using a liposome floatation assay, where we incubated liposomes generated from E. coli polar lipid extract with a recombinant MBP-ahBrl1-yEGFP fusion protein (Figure 5E). We observed that MBPahBrl1-yEGFP was enriched in the floating fraction, whereas fusion proteins that carry single point mutations disrupting the hydrophobic face of ahBrl1 (F391D and I395D) displayed strongly reduced liposome binding compared to the negative control MBP-TEV-yEGFP (Figure 5E). Interestingly, we observed that an ahBrl1-yEGFP fusion protein expressed in yeast cells was enriched at the NE in vivo

364 (Figure 5F). Together, these results demonstrate that ahBrl1 can bind to lipid membranes in vitro and in vivo and is essential for cell viability. 

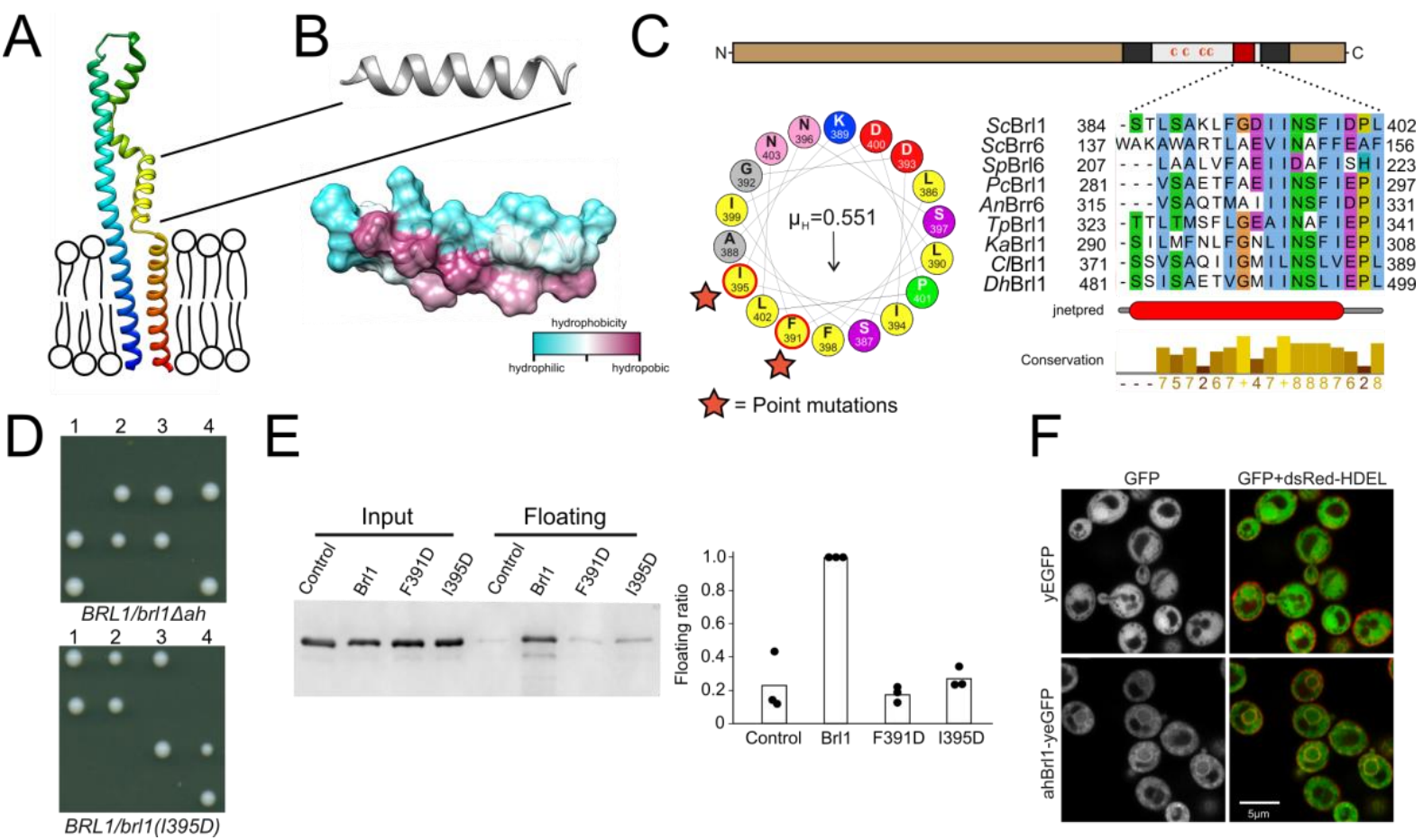

$\mathrm{F}$

Figure 5: A conserved luminal amphipathic helix binds to membranes and is essential for Brl1 function. A)

AlphaFold prediction for Brl1 (Jumper et al. 2021). Unstructured termini are not shown; blue: N-terminus, red: C-terminus. Transmembrane domain highlighted by the lipid bilayer. B) Predicted amphipathic helix in ribbon and surface representation, colored based on hydrophobicity. C) Upper panel: Domain architecture of Brl1: extraluminal $\mathrm{N}$ - and C-terminus in brown, transmembrane domains in dark gray, amphipathic helix in red; Left panel: Helical wheel representation of the amphipathic helix of Brl1 and the hydrophobic moment determined with HeliQuest (Gautier et al. 2008). Point mutants are indicated by stars. Right panel: Conservation and secondary structure prediction of the amphipathic helix in different fungi. Hydrophobic: blue, negative: magenta, polar: green, glycine: orange, proline: yellow, unconserved: white. Jnetpred4 secondary structure prediction (Drozdetskiy et al. 2015): helices are marked as red tubes. Sc: Saccharomyces cerevisiae, Sp: Schizosaccharomyces pombe, Pc: Pneumocystis carinii, An: Aspergillus nidulans, Tp: Tetrapisispora phaffii, Ka: Kazachstania Africana, Cl: Clavispora lusitaniae, Dh: Debaryomyces hansenii. D) Vertically oriented tetrad offspring of heterozygous Brl1 mutants carrying one allele lacking the amphipathic helix (brll $\Delta a h$ ) or a single point mutation in the hydrophobic side of the helix ( $\operatorname{brl}(1395 D))$. E) Membrane floatation assay with purified MBP-ahBrl1(377-406)-yEGFP fusion proteins and liposomes made of E. coli polar lipids extract. Control: MBPGFP. Mean of three biological replicates, individual data points are indicated. F) Coexpression of yEGFP or ahBrl1-yEGFP from the GAL1 promoter with the ER/NE marker dsRed-HDEL. 

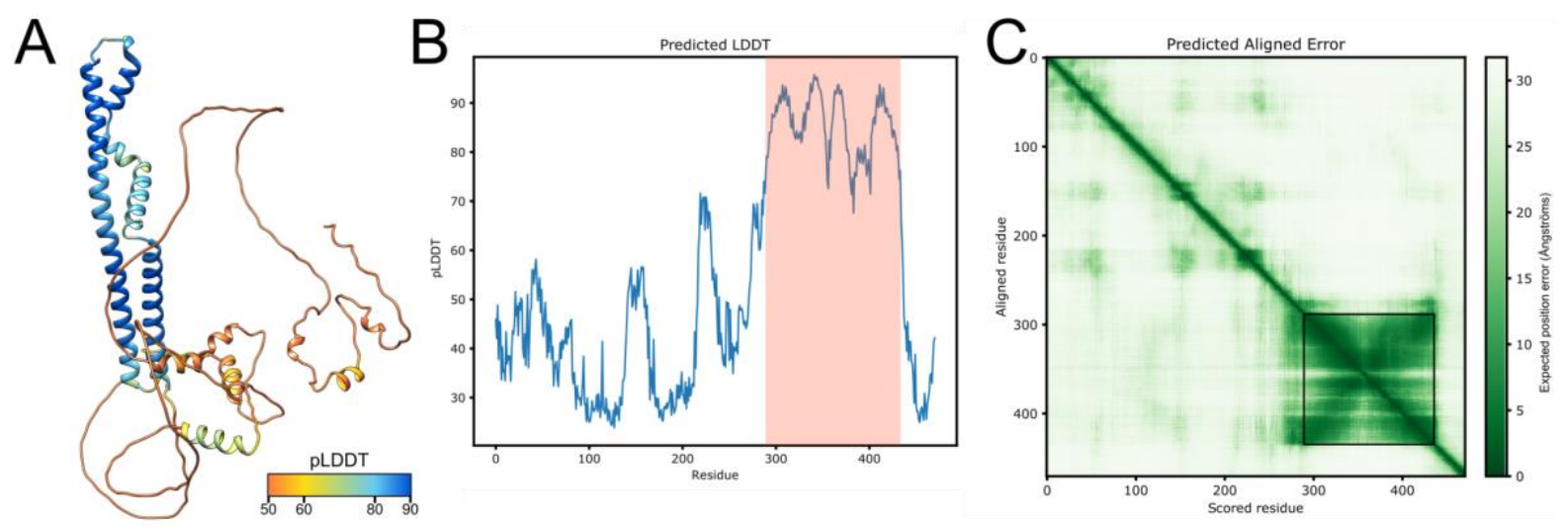

D

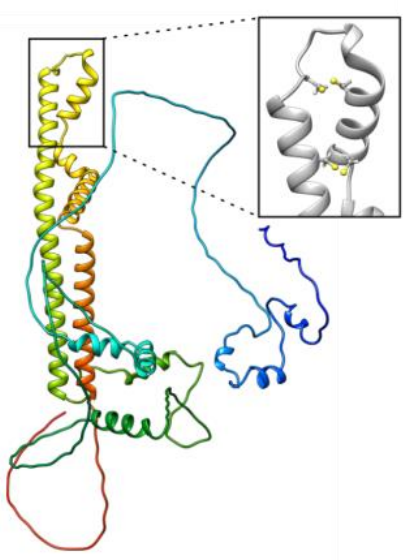

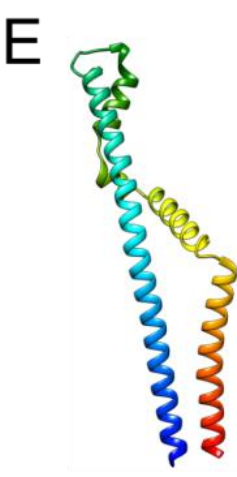

$\mathrm{F}$

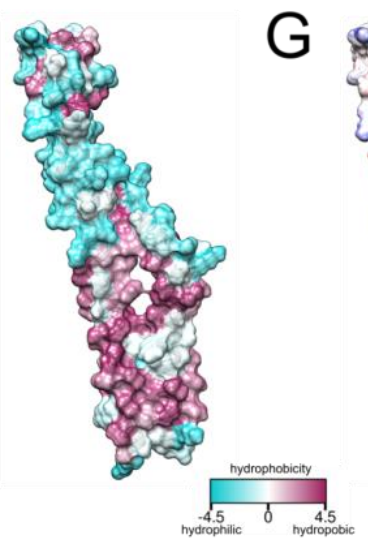

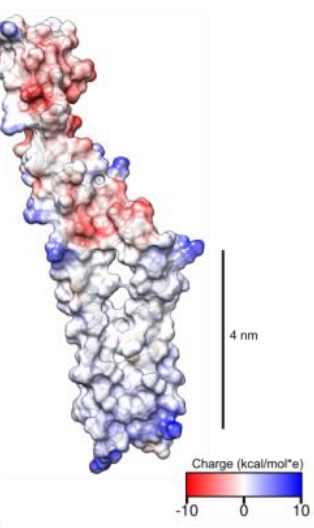

Figure S4: AlphaFold structure prediction for Brl1 A) Predicted structure for full-length Brl1 using

AlphaFold2, colored based on the predicted local distance difference test score (pLDDT). B) pLDDT score for Brl1. High confidence region shown in Figure S4E-G and Figure 5A indicated by red frame. C) Predicted aligned error for Brl1, dark green area indicates high inter-domain accuracy. High accuracy region shown in Figure S4E$\mathrm{G}$ and Figure 5 is highlighted by the black frame. D) Predicted structure for Brl1, colored in rainbow (red: Cterminus, blue: N-terminus), inset shows the position of the 4 cysteines in the luminal domain of Brl1. E) Predicted structure for high confidence region Brl1(289-434) in rainbow coloring (red: C-terminus, blue: N-terminus). FG) Surface representation of Brl1(289-434), colored by hydrophobicity (Kyte-Doolittle scale) S4F and charge S4G. 
Overexpression of Brl1(I395D) blocks NPC maturation and leads to herniating INM sheets at NPC assembly sites

398 Since ahBrl1 is required for Brl1's function we wanted to elucidate its role during NPC assembly. Previously, it was reported that overexpression of Brl1 bypasses the requirements for Nup116 and Gle2 in NPC biogenesis (Liu et al. 2015; Zhang et al. 2018). We screened the effect of six single point mutations in ahBrl1 for the ability to rescue growth of the nup1164GLFG $P_{M E T 3}-N U P 188$ strain (Figure S5A). We observed that overexpression of Brl1 mutants, replacing the hydrophobic residues F391, I395, F398 or L402 by the charged aspartic acid not only failed to rescue the assembly defect but had a dominant negative effect on cell growth (Figure 6A and S5A). When residues at the polar side of the helix (D393 and D400) were substituted to alanine, functionality was not perturbed (Figure S5A). The dominant negative growth inhibition persisted in the wild-type background (Figure 6A), demonstrating that overexpression of Brl1 with an impaired $\mathrm{AH}$ alone is toxic.

To understand the causes of the dominant negative effect of ahBrl1 mutant overexpression, we examined the localization of yEGFP-fused Brl1, Brl1 $\Delta$ ah and Brl1(I395D) expressed under a galactoseinducible promoter (Figure 6B). Brl1 $\Delta$ ah and Brl1(I395D) initially localized to the NE-ER network, occasionally forming bright foci at the NE. However, after six hours of expression most of the protein was localized in large NE accumulations (Figure 6B). In contrast, overexpression of Brl1 with an unperturbed AH uniformly localized to the NE and the ER (Figure 6B), as also shown previously (Saitoh et al. 2005; Zhang et al. 2018). Since wild-type Brl1 is unable to fulfill its function upon overexpression of the ahBrl1 mutants, we also wanted to analyze the localization of the endogenous copy of Brl1 in these conditions. Interestingly, we found that yEGFP-tagged Brll colocalized with the large BrlI395DmCherry puncta at the NE (Figure S5B). This suggests that a sequestration of endogenous Brl1 to these accumulations could potentially lead to the dominant negative effect of the ahBrl1 mutants and that a critical concentration of Brl1 with a functional AH is needed for successful membrane fusion at NPCassembly sites. The dominant negative growth defect of overexpressed ahBrl1 mutants could thus be caused by the formation of toxic assemblies, which also trap the endogenous Brll protein. To test whether Brl1(I395D) can dynamically exchange between NE accumulations or is trapped there, we probed the dynamics of Br11(I395D)-mCherry at the herniations with Fluorescence Recovery After Photobleaching (FRAP) (Figure 6C). We co-expressed either Brl1-mCherry or Brl1(I395D)-mCherry with Sec61-yEGFP, a transmembrane protein, that can freely diffuse between the ER/ONM and the INM (Deng and Hochstrasser 2006; Popken et al. 2015). We compared the fluorescence recovery of Brl1-mCherry with Sec61-yEGFP in an arbitrary NE region and saw that both proteins fully recover with a comparable half-life $\left(\tau_{1 / 2}\right)$ of $\sim 2$ seconds, indicating that they freely diffuse in the membrane of the NE (Figure 6C). This is in line with our lysis intermixing experiments, where we saw that Brl1 
Supplementary Results Figure 1B-D). Next, we photobleached the fluorescent signal of Brl1(I395D)mCherry and Sec61-yEGFP in the NE-attached foci and observed that Brl1(I395D)-mCherry has a high immobile fraction that is not replaced over the time scale of 25 seconds, while Sec61-yEGFP almost fully recovered (Figure 6C). The $\tau_{1 / 2}$ of recovery of the mobile fraction of Brl1(I395D)-mCherry is comparable to Brl1-mCherry. These data suggest that passage of Brl(I395D)-mCherry through the NPC intermediate structure is an irreversible process, and once Brl1(I395D)-mCherry reaches the herniations it likely multimerizes and is trapped.

438 We next wanted to test if the NE accumulations of Brl1(I395D)-mCherry also trap NPC components.

439 To this end, we analyzed the colocalization of Brl1(I395D)-mCherry with several yEGFP-tagged Nups:

440 Nup116, Nup133 and Nup170 display regular NE localization and importantly, can be detected in the 441 NE-regions corresponding to the Brl1(I395D)-mCherry foci (Figure 6D). In contrast, Nup82 is entirely 442 absent from NE-areas with Brl1(I395D)-mCherry puncta. This labeling pattern is consistent with the one observed in the NPC herniations that form upon Brl1 depletion (Figure 3 and 4) suggesting that overexpressed Brl1(I395D) concentrates adjacent to NPC assembly intermediates composed of the IR and Y-complex but not the cytoplasmic NUPs.
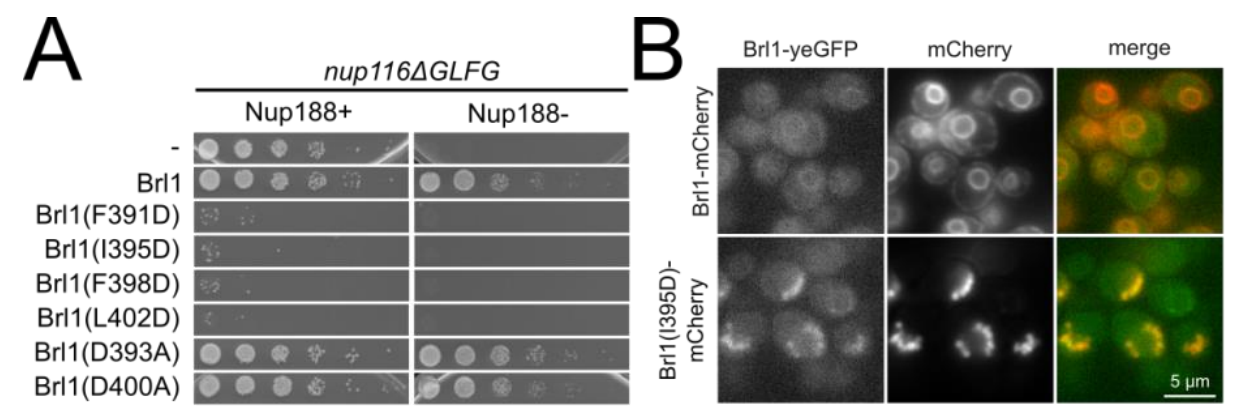

Figure S5: Luminal AH of Brl1 is involved in NPC biogenesis. A) Spotting assay of five-fold serial dilutions of nup 116 GLFG PMET3-NUP188 cells expressing various ahBrl1 domain mutants from the GAL1 promoter. B) Co-localization of mCherry tagged Brl1 or Brl1(I395D) expressed from a galactose-inducible promoter with endogenously tagged Brl1-yEGFP. Lower panel: Maximum intensity plots of Brl1 (green line) relative to maximum Brl1(I395D)-mCherry signal in NE foci (red line) from nucleoplasm (left) to cytoplasm (right). 
bioRxiv preprint doi: https://doi.org/10.1101/2022.03.04.483005; this version posted March 4, 2022. The copyright holder for this preprint

(which was not certified by peer review) is the author/funder, who has granted bioRxiv a license to display the preprint in perpetuity. It is made available under aCC-BY-NC-ND 4.0 International license.

Average and standard deviation for 55 line-plots, every point is an average of $n>30$ values. A representative image used for analysis is shown in inset.

A

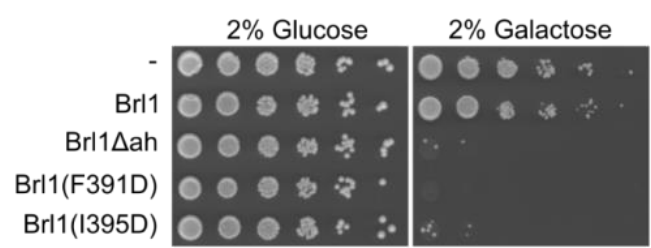

C
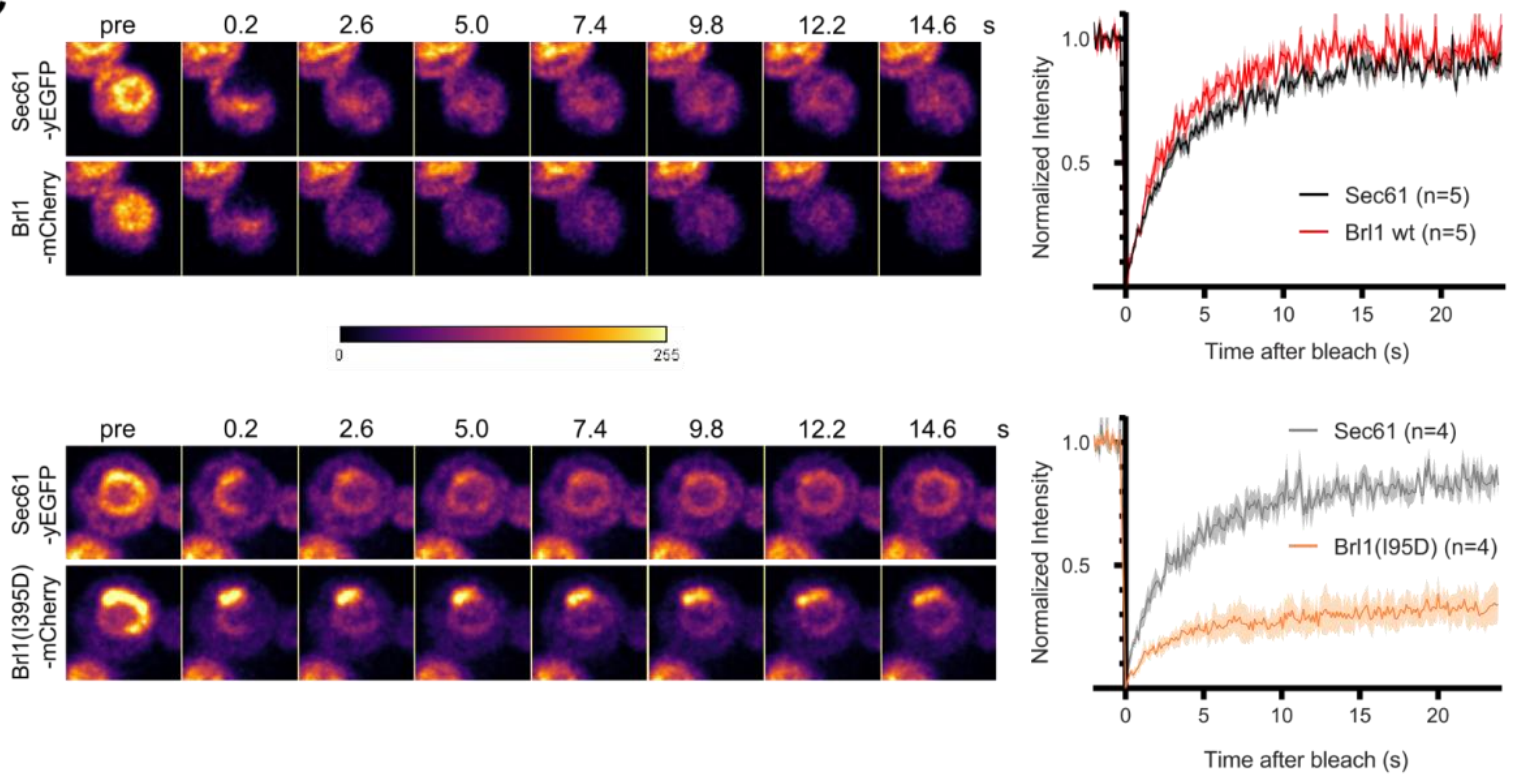

$\mathrm{D}$
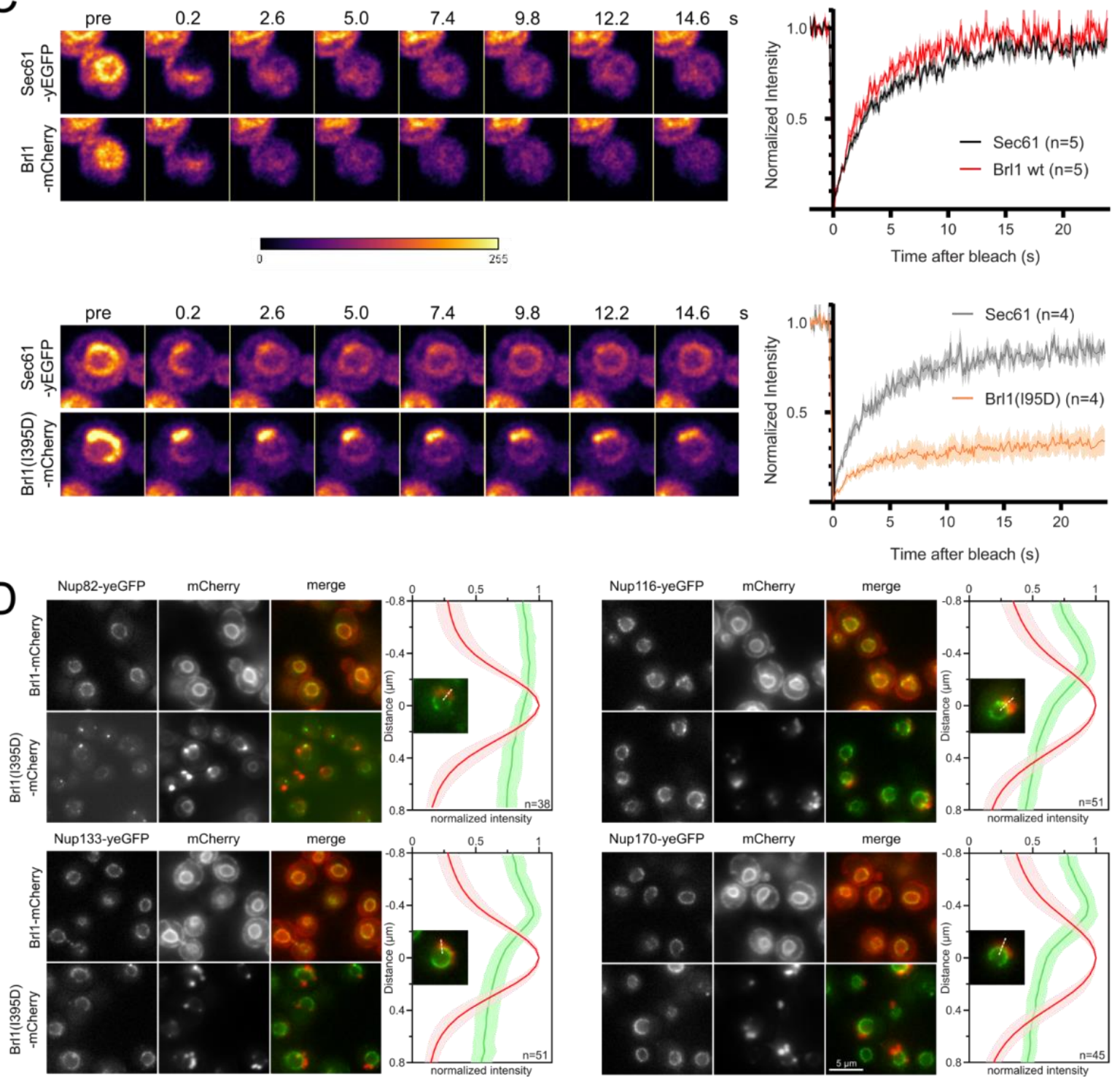

Figure 6: Overexpression of Brl1(I395D) with an impaired amphipathic helix interferes with NPC

assembly. A) Spotting assay of wild-type cells expressing Brl1, Brl1 $\Delta$ ah or Brl1(I395D) from the GAL1 promoter 
the GAL1 promoter in SD 2\% galactose. Brightness contrast settings of nuclei in insets are adjusted differently.

C) Fluorescence Recovery After Photobleaching of Sec61-yEGFP, Brl1-mCherry and Brl1(I395D)-mCherry. Left panels: representative images of recovery, right: corresponding averaged recovery curves(n>4). One representative experiment of three biological replicates is shown. Images are shown in pseudocolor. D) Colocalization of mCherry tagged Brl1 or Brl1(I395D) and yEGFP-tagged NUPs: mCherry channel is scaled differently between images. Maximum intensity plots of NUPs (green lines) relative to maximum Brl1(I395D)mCherry signal in NE foci (red line) from cytoplasm (bottom) to nucleoplasm (top). Average and standard deviation of more than 38 line-plots with $n>31$ values averaged for each point. A representative image used for the analysis is shown for each condition in inset.

To gain ultrastructural insights into the organization of the Brl1(I395D) accumulations, we investigated cells using cryo-ET on FIB-milled lamella (Figure 7A-7C, Movies S5 and S6). We observed mature NPCs, INM evaginations and NE herniations as already seen in Brl1-depleted cells (Figure 7A panel iiii, Figure 7B and Movie S5). No herniations could be observed in control cells (Figure 7B, movies S7 and S8). To our surprise upon Brl1(I395D) overexpression, we also found large multi-layered herniations with diameters up to $\sim 600 \mathrm{~nm}$, so far not reported in any other NPC assembly mutant (Figure 7A panel iv-vi, Movie S5 and S6). These onion-like structures are composed of elongated INM herniations curling over each other with up to four stacked double bilayers. Of note, inter-membrane distances were remarkably constant with two discrete widths of the innermost sheets, suggesting two different maturation modes for the onion-like herniations (Supplementary results: "Model for the development of "onion-like" herniations"). Unlike the herniations in Brl1-depleted cells (Figure 2A), these structures were not filled with electron-dense material and only occasionally enclosed small patches of aggregate-like densities (Figure 7A panel v-vi, movies S5 \& S6). Single subtomograms and the subtomogram average of 47 herniations confirm the presence of an NPC intermediate with a diameter of $97 \mathrm{~nm}$ at the bases of these herniations (Figure 7C). Densities which likely correspond to the IR and the nucleoplasmic Y-complex ring but not the cytoplasmic side of the NPC can be distinguished. Although our average did not allow for unambiguous assignment or structure fitting, these densities look similar to the structures we observed in herniations of Brl1-depleted cells (Fig S2D ii) and the previously reported herniation structure in nup 1164 cells at $37^{\circ} \mathrm{C}$ (Allegretti et al. 2020), and are in a good agreement with the NUP localization patterns observed by fluorescence microscopy (Figure 3C-3F).

493 Altogether, these results demonstrate the critical role of Brl1's AH during NPC maturation. The fact 494 that the essential luminal ahBrl1 has a propensity to bind membranes, and the observation that Brl1 acts 495 prior to INM-ONM fusion suggests that Brl1 acts as a fusogen with membrane deforming properties. 496 By deforming the INM, Brl1 could assist in the last NPC maturation step: the formation of a nucleo497 cytoplasmic transport channel. 

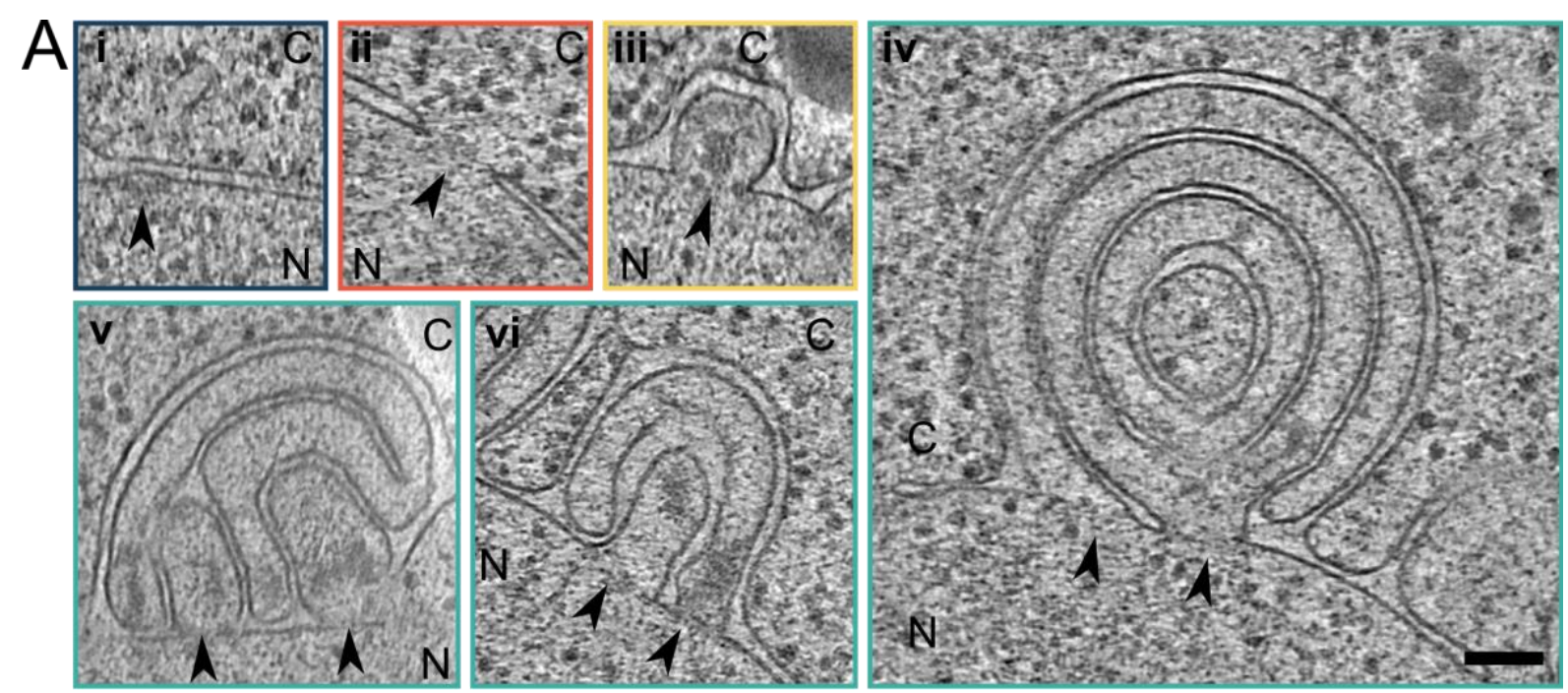

B
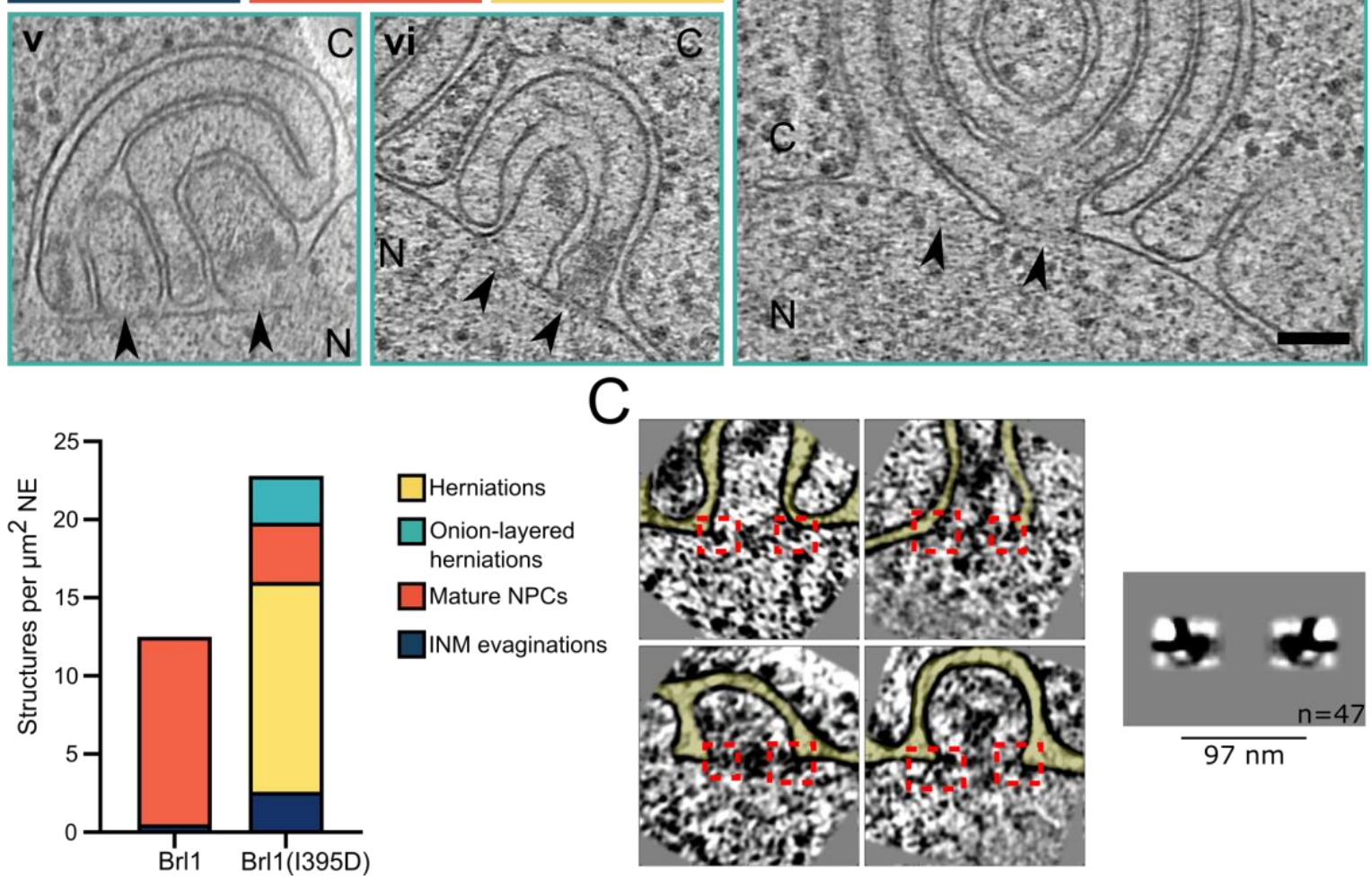

$97 \mathrm{~nm}$

Figure 7: Br11(1395D) overexpression leads to the formation of multi-layered NE herniations. A)

Tomographic slices of the NPC-like structures quantified in 7B, observed in FIB-milled cells overexpressing Brl1(I395D), scale bar: $100 \mathrm{~nm}, \mathrm{~N}$ : nucleus, C: cytoplasm, slice thickness: $2.1 \mathrm{~nm}$, arrows indicate NPC-like structures. Image frames colored according to the color code used in 7B. Panels iv and vi are tomographic slices from the tomogram in movie S5. B) Quantification of observed structures in Brl1(I395D) cells and control condition; $17\left(5.1 \mu \mathrm{m}^{2} \mathrm{NE}\right)$ and $50\left(9.8 \mu \mathrm{m}^{2} \mathrm{NE}\right)$ tomograms were quantified for cells overexpressing Brl1 or Brl1(I395D) respectively. C) Single subtomograms and subtomogram average of 47 herniations in Brl1(I395D) overexpressing cells; box size of subtomograms is $270 \mathrm{~nm}$; cytoplasm is at the top in each image. 


\section{Discussion}

508 The NPC is one of the largest cellular protein complexes, yet only few non-NPC proteins have been suggested to aid with its biogenesis. One such factor is the integral membrane protein Brl1. However, the timing of Brl1-function in the NPC assembly process or mechanistic details of its action have remained elusive. In this study, we show that Brl1 is essential for NPC biogenesis, and we provide functional insight into its role in membrane fusion.

513 Based on its binding capacity to structural NUPs, it was previously proposed that Brl1 associates with 514 NPC maturation intermediates (Zhang et al. 2018). Using our recently developed KARMA method 515 (Onischenko et al. 2020), we now demonstrate that Brl1 indeed preferentially interacts with newly synthesized NUPs and in addition, we found that Brl1 primarily co-localizes with newly produced nucleoporin assemblies in cells (Figure 1D-1F). Furthermore, functional inactivation of Brl1 stalls NPC assembly without affecting previously assembled NPCs (Figure 3E-3F). This leads to the accumulation of NE herniations that have a continuous ONM and contain incompletely assembled NPCs lacking the cytoplasmic export platform (Figure 3-4 and S2). Thus, our results clearly identify Brl1 as an NPC assembly factor.

Depletion of Brl1 leads to the formation of incomplete NPC structures that contain the IR, membrane ring, Y-complex and nuclear basket NUPs. The cytoplasmic Nup159 and Nup82 are absent from the intermediates but instead are mislocalized in cytoplasmic foci, as seen previously in other NPCassembly mutants (Hodge et al. 2010; Makio et al. 2009; Onischenko et al. 2009, 2017; Scarcelli et al. 2007) (Figure 3C-3D). In light of the observed NE herniations in Brl1-depleted cells (Figure 3A-B and $\mathrm{S} 2 \mathrm{E}$ ), the fusion of the INM and ONM appear to be a prerequisite for the recruitment of the cytoplasmic Nup159-Nup82-Nsp1 complex. Thus, our data support an inside-out mode of interphase NPC assembly, similar to previously proposed models in yeast and mammalian cells (Onischenko et al. 2020; Otsuka et al. 2016; Thaller and Patrick Lusk 2018). Interestingly, in Brl1-depleted cells the Y-complex NUPs display a reduced NE fluorescence signal and slow fractional labeling in our proteomic assays (Figure 3C-D and 4C-D). This suggests that only the nucleoplasmic Y-complex ring is present in the intermediates. This is also in line with our cryo-EM data (Figure S2E) and with previous results in nup116 cells (Allegretti et al. 2020) suggesting that INM-ONM fusion is needed before the cytoplasmic Y-ring can be recruited to the assembling NPC.

536 We also observed that halted NPC assemblies accumulating upon Brl1 depletion contain Mlp1 (Figure

537 3C-F and 4C-D). In native NPC biogenesis, the nuclear basket NUPs and especially Mlp1 join very late 538 (Onischenko et al. 2020). Interestingly, the slow metabolic labeling of Mlp1 in Brl1 depleted cells shows that it still assembles late, however, unlike the cytoplasmic export platform NUPs, Mlp1 is not blocked from incorporation. This indicates that Mlp1 is recruited independently from Brl1 in a 
possible to reconstitute a nuclear basket scaffold that contains Nup60, Nup2 and Mlp1 in absence of any other NUPs in vitro (Cibulka et al. 2022). This highlights that NPC biogenesis is likely not a strictly hierarchical process.

The fusion of INM and ONM is a crucial step during de novo NPC assembly in interphase. Membrane fusion does not occur spontaneously, and based on previously characterized membrane fusion events, it is likely that two NE lipid bilayers must be brought into proximity to initiate the fusion of the membranes (Peeters, Piët, and Fornerod 2022). While the fusion event itself is expected to be fast and thus difficult to investigate, potential assembly-intermediate states in which INM and ONM approach each other but are not yet fused, can be observed in cells with NPC-assembly defects (Makio et al. 2009; Thaller and Patrick Lusk 2018) and rarely also in normal cells (Otsuka et al. (Otsuka et al. 2016) and our cryo-ET data (Movie S3, figure 3B and 7B)). It has been suggested that NUPs and other proteins containing amphipathic helices are important players in the formation and stabilization of these early NPC-intermediates since they can bind to and deform membranes (Dawson et al. 2009; Jakub et al. 2022; Schooley et al. 2012; Voeltz et al. 2006; Wang et al. 2021). In this study, we identified a membrane-binding amphipathic helix (AH) within the luminal domain of Brl1 which is essential for its function in NPC assembly, as genetic perturbations that abolish membrane binding lead to severely impaired NPC biogenesis. Interestingly, this AH is highly conserved in organisms with closed mitosis and is a shared feature of proteins associated with NPC assembly such as Brr6, Apq12 and ER-bending reticulons (Dawson et al. 2009; Zhang et al. 2021). Taken together, these results emphasize the emerging role of AH motifs in NPC assembly.

Brr6 is a paralogue of Brl1 with the same topology and orientation in the NE. Interestingly, Brr6 also contains a predicted luminal amphipathic helix, indicating that both proteins might function similarly. Further, it has been shown that Brr6 co-localizes at Brl1-foci at the NE and physically interacts with Brl1 (Lone et al. 2015; Saitoh et al. 2005; Zhang et al. 2018). However, deletion of Brl1 or Brr6 cannot be rescued by overexpression of the respective paralogue and several NPC-assembly mutants such as gle2A, nup1164 and nup1164GLFG $P_{M E T 3} N U P 188$ can only be rescued by Brl1-overexpression. This demonstrates that despite similar sequence ( $44 \%$ sequence similarity of the structured parts) and structure, Brl1 and Brr6 do not act redundantly in NPC assembly. This is also in agreement with the differential localization of these two proteins: Brl1 mainly localizes to the INM whereas Brr6 can be found in both NE leaflets (Zhang et al. 2018). Thus, it seems likely that Brl1 and Brr6 act in concert during NPC-assembly and membrane fusion, however, the detailed function of Brr6 and the role of additional NUPs and potential assembly factors like Apq12 remains unclear and awaits further characterization.

575 How does Brl1 promote interphase NPC assembly? Our observations that NPC-assembly intermediates that form in the absence of Brl1 already contain membrane-binding NUPs (Figure 4D) suggest that they 
that Brl1 is recruited to and concentrated at these NPC-assembly sites (Figure 8A). This view is supported by the punctate localization pattern of endogenously tagged Brl1-yEGFP (Lone et al. 2015), co-localization of Brl1-puncta with newly synthesized NUPs (Figure 1E) and accumulation of dysfunctional Brl1 mutants at stalled NPC assembly sites (Figure 6B, 6D and figure 7). The mechanisms by which Brl1 is recruited and to concentrated at assembly sites is not clear but could be achieved by the unstructured $\mathrm{N}$-terminus of Brl1 or alternatively via the localization preference of Brl1 to the curved membranes of INM evaginations. The former possibility is supported by the non-punctate localization of Brr6 which contains only a short N-terminus (Lone et al. 2015). Irrespective, it seems likely that a high local concentration of Brl1 is critical for membrane fusion as overexpression of Brl1 can rescue assembly defects in multiple NUP mutants (Liu et al. 2015; Zhang et al. 2018).

Our results show that ahBrl1 is required for the INM-ONM fusion event since cells that express Brl1 with an impaired $\mathrm{AH}$ are not viable and overexpression of Brl1(I395D) inhibits NPC biogenesis leading to the formation of NPC-assemblies with multi-layered INM herniations (Figure 7A). Brl1(I395D) accumulates and multimerizes in these structures as shown by the high concentration and slow mobility in herniations (Figure 6B and 6C). Since overexpressed Brl1(I395D) strongly accumulates at the assembly sites and induces the formation of highly curved onion-like membrane sheets, we speculate that in the absence of a functional AH, Brl1 can still mediate membrane remodeling but not INM and ONM fusion. This points to an important role of ahBrl1 in the membrane fusion event (Figure 8C) but how could Brll and its AH mediate the fusion of the INM and ONM? Interestingly, the predicted structure of Brl1 reveals the presence of a luminal, $~ 8 \mathrm{~nm}$ long continuous alpha-helix (Figure 5A) that is stabilized by disulfide bridges (Figure S4D) (Zhang et al. 2018). Whereas this helix is too short to span the entire $\sim 21 \mathrm{~nm}$ of the NE lumen (Supplementary results figure $2 \mathrm{~B}$ ), it is conceivable that at INM-herniations, where the two leaflets approach each other, this helix could interact with proteins in the ONM (Figure 8D). Intriguingly, a similar, long helix is also predicted in Brr6. It is tempting to speculate that Brl1 at the INM interacts with Brr6 at the ONM at early NPC assembly sites and that this interaction leads to INM-ONM fusion mediated by the conserved AHs present in both proteins. This possibility is supported by the differential localization patterns of Brl1 and Brr6 in immunogold labeling assays wherein Brl1 predominantly localizes at the INM while Brr6 is equally distributed between INM and ONM (Zhang et al. 2018). 
A

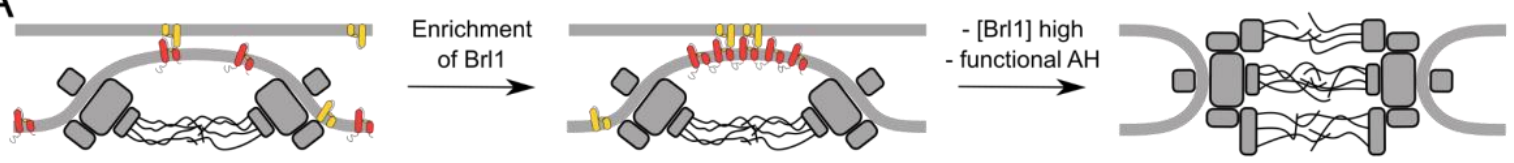

B
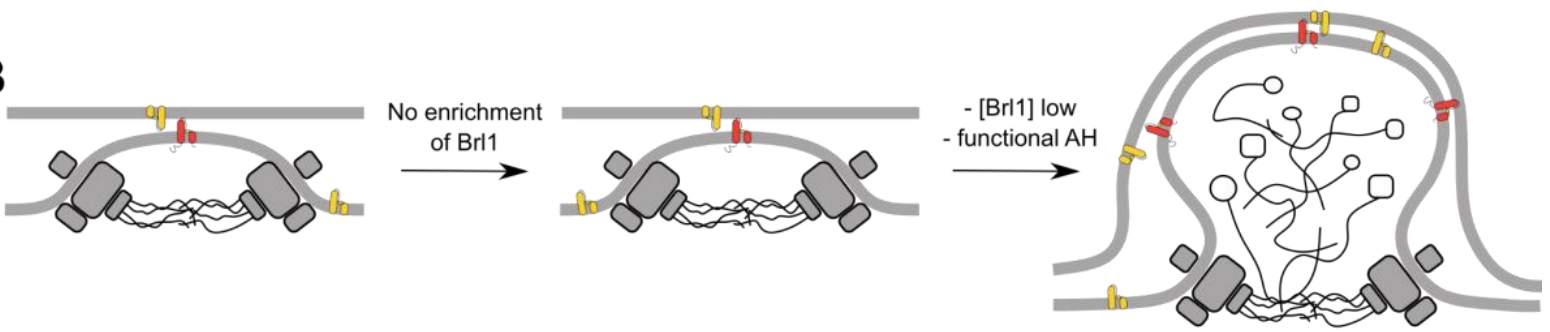

C
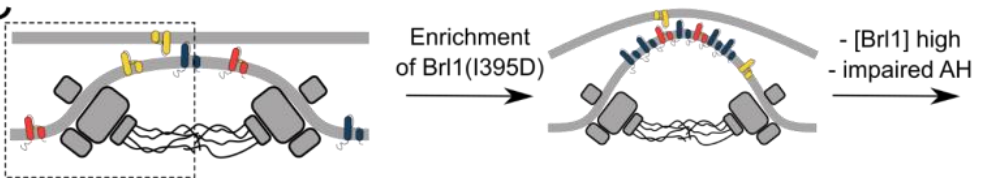

D
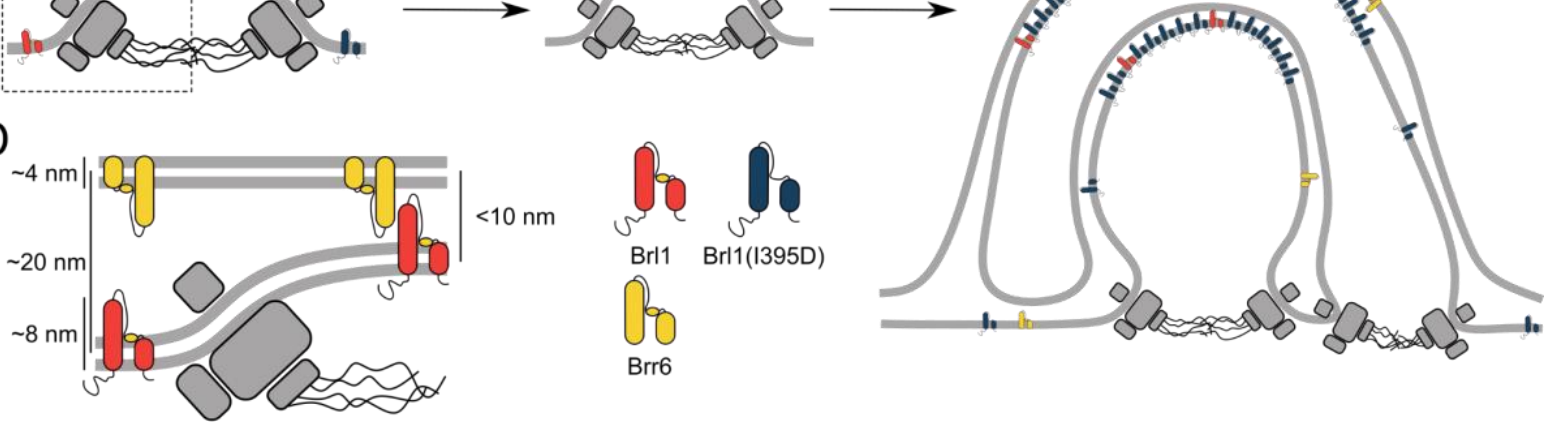

Figure 8: The role of Brl1 during NPC assembly. A) Brl1 (red) enriches on the inside of NPC maturation intermediates and promotes INM-ONM fusion through the membrane-binding AH motif and likely in cooperation with Brr6 (yellow). B) If Brl1 cannot reach the critical concentration required to promote membrane fusion, unresolved NE herniations, filled with electron dense material, appear. C) Overexpression of Brl1(I395D) with a perturbed AH (blue) concentrates at the NPC assembly site. It remodels the NE membranes and leads to expanded multi-layered herniations but ultimately fails to induce membrane fusion. D) Brl1 at the INM can only physically interact with Brr6 or Brll at the ONM when the NE leaflets approach, as it is the case at NPC-assembly sites. Dimensions based on our cryo-ET data (Figure S2D), structure prediction (Figure S4) and measurements of the NE (Supplementary results figure 2B).

Aside from the direct role in membrane fusion, Brl1 might also affect the lipid composition of the NE. Indeed, it has been proposed that Brl1 forms a sensory complex with Brr6 and Apq12 that controls membrane fluidity (Lone et al. 2015). During NE-fusion and other NPC-assembly steps, the membrane curvature of the NE is extensively modulated and changes in lipid composition, either globally or locally at NPC assembly sites, could facilitate this process. In fact, in Apq12 overexpressing cells, phosphatidic acid (PA) accumulates at sites of ONM-overproliferation (Romanauska and Köhler 2018; Zhang et al. 2021). A similar PA accumulation was reported at nup 1164 herniations, indicating that PA might be a relevant effector during NPC-assembly (Thaller et al. 2021). However, the effects of Brl1, Brr6 and Apq12 on lipid composition are somewhat controversial (Lone et al. 2015; Scarcelli et al. 2007; Zhang et al. 2018) requiring better tools to understand the role of lipid environment in NPC biogenesis. Of 
628 note, membrane proliferation or remodeling can also be induced by an overexpression of membrane 629 proteins without necessarily altering the overall lipid composition. For example, overexpression of 630 transmembrane proteins induces the formation of karmellae (Wright et al. 1988), expansions of the 631 NE/ER membranes. Similarly, overexpression of AH-containing NUPs was shown to induce NE 632 overproliferation resulting in multiple, stacked membrane cisternae (Marelli et al. 2001; Mészáros et al. 633 2015) that were also observed upon overexpression of Brl1 or Brr6 (Zhang et al. 2018). Therefore, it is 634 likely that NE-overproliferation also plays a role in the generation of the onion-like herniations that we 635 observe in cells overexpressing the dominant-negative Brl1 variant, Brl(I395D). In the future, it will be 636 important to manipulate NE lipids and to characterize the effects of membrane composition in NPC637 assembly. 


\section{Supplementary Results}

640 Analysis of protein labeling in source lysate

641 To ensure that the observed differences in labeling kinetics in the Brl1 KARMA assay are not the result 642 of differences in protein turnover, we assessed the labeling of several proteins in the source lysate of 643 the APs by parallel reaction monitoring mass spectrometry (Peterson et al. 2012). As expected, both 644 NTRs and two randomly picked abundant co-purified proteins showed essentially the same metabolic 645 labeling in the source cell lysates and the corresponding APs (Supplementary results Figure 1A). NUPs 646 from different assembly tiers did not show a systematic labeling difference in the source lysate, as was 647 the case for the AP (Supplementary results Figure 1A). This shows that the kinetic differences are 648 specific to the Brl1 AP.

\section{Lysis intermixing assay}

650 A factor that could influence the labeling kinetics measured in KARMA assays is dynamic protein 651 exchange during the AP procedure (Tackett et al. 2005). To test for the significance of this effect, we 652 quantified the metabolic labeling in AP fractions of equal mixes of wild-type culture grown in heavy 653 lysine medium and a Brl1 affinity tagged strain grown in light lysine medium (Supplementary results 654 figure S1B). Strikingly, we found that all NUPs readily intermix during the AP procedure to more than $65580 \%$ (Supplementary results figure S1C), values far higher than what we previously observed with a 656 stably bound NUP bait ( 20\% with Mlp1) (Onischenko et al. 2020). Such a high extent of intermixing 657 suggests that the Brl1 association with NPCs is likely very dynamic. Interestingly, we also observed a 658 pronounced negative correlation between NUP metabolic labeling in KARMA assays and the 659 intermixing tests (Supplementary results Figure 1D), suggesting that Brl1 binds young nucleoporin 660 assemblies more stably.

\section{$661 \quad$ Kinetic state model}

662 The high labeling rates in KARMA assays with the Brl1 bait (Figure 2B) and the in vivo fluorescence 663 microscopy (Figure 1D-1E) both indicate that Brl1 preferentially binds to young NPC assemblies. In 664 the lysis intermixing tests we found that Brl1 interacts with the NPCs highly dynamically 665 (Supplementary results figure S2B-S2D) and likely also loosely binds to mature structures. Consistent 666 with this, we still detect intermediate and late NUPs in Brl1 AP fractions (Figure S1B). To assess the 667 binding preference of Brl1 during NPC assembly in a quantitative manner we made use of the three668 step KSM that we have previously developed (Onischenko et al. 2020). Note, the KSM that was 669 originally designed to account for completely inaccessible pools of mature NPCs (e.g., ones that are 670 sequestered and cannot be pulled down), but in the context of Brl1, these pools have a new meaning 
reflecting the lower affinity of Brl1 to late complexes. Our KSM analysis revealed that a considerable

672 fraction of primarily early tier NUPs become inaccessible to Brl1 bait (Figure 2C). The smaller

673 inaccessible pools of late and intermediate NUPs indicate that a fraction of the Brll dissociates prior to

674 their assembly. By contrast, NUP baits almost never led to inaccessible pools, consistent with them

675 being constitutively bound and not leaving the NPC once assembled (Figure 2C).

676

A

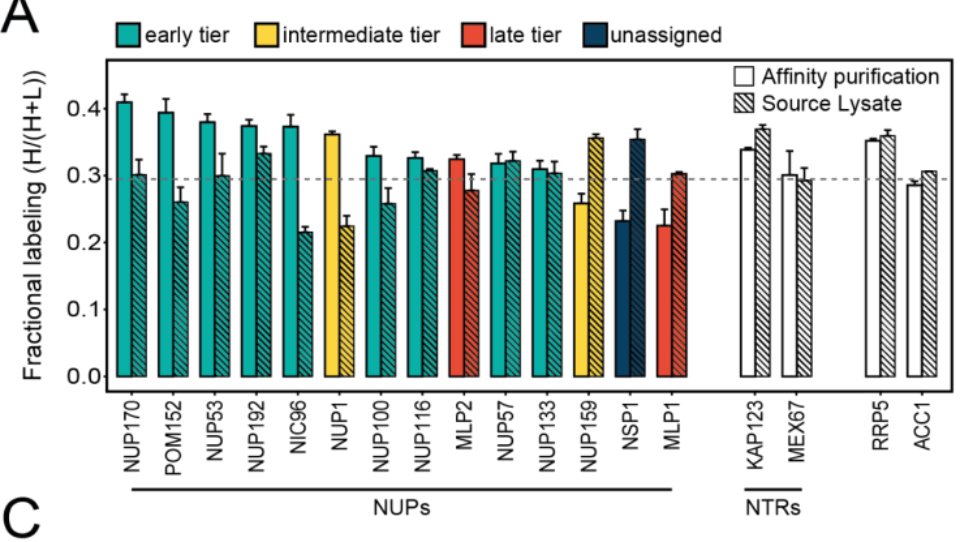

677

678

679

680

681

682

683

684

685 tier. Median of three biological replicates each.
B

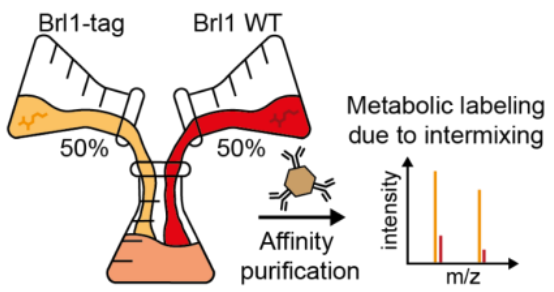

D

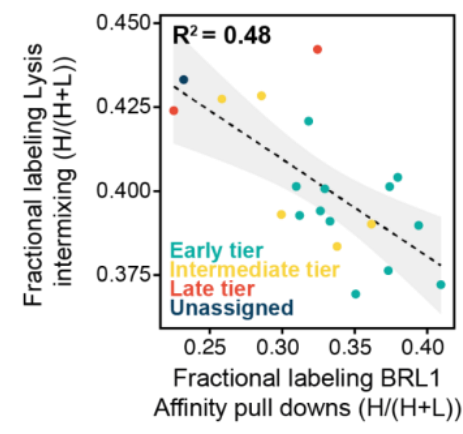

Supplementary results figure 1: Control protein labeling in KARMA assays. A) Fractional labeling of NUPs and NTRs in KARMA assays with Brl1 bait and the respective source lysate, 90 min after labeling onset. Median $\pm \mathrm{SD}$ of three biological replicates. B) Experiment to test the intermixing dynamics. Equal fractions of an unlabeled Brl1 affinity tagged strain and a wild-type culture grown in labeled medium were subjected to the affinity purification procedure. C) Percentage of intermixing for NUPs and NTRs normalized to the mean of all co-purified proteins. Median \pm SD of three biological replicates. D) Correlation of NUPs between fractional labeling in the intermixing experiment and in KARMA assay with Brl1 bait. Coloring according to the assembly 


\section{Model for the development of "onion-like" herniations}

687 The large multi-layered "onion-like" herniations that form in response to Br11(I395D) overexpression,

688 have not been reported before and the question arises of how these structures could assemble at the NE.

689 Interestingly, we noticed a remarkably constant spacing between the two bilayers and the enclosed

690 nuclear space. Morphometric analysis of the different lipid layers reveals that the middle sheets

691 consisting of two INMs have a very regular spacing of $\sim 13 \mathrm{~nm}$ (Supplementary results figure 2A-B).

692 The intermembrane spacing in the outer layer consisting of INM and ONM is significantly wider ( 19

$693 \mathrm{~nm})$, which is very similar to the spacing of regular NE in our control condition ( 21 nm). Interestingly,

694 the innermost layers show a bimodal distribution with two peaks at heights of the INM-INM middle

695 layers and the INM-ONM outer layers (Supplementary results figure 2A-B). This could be explained

696 by two distinct maturation mechanisms of the onion-like structures. In maturation mode 1, an elongated

697 herniation curls around a part of the cytoplasm and further grows until membrane fusion leads to the

698 enclosure of cytoplasm in the very center of the herniation. Growth and fusion events of subsequent

699 herniations then result in the multi-layered herniations (Supplementary results figure 2C). Consistent

700 with this mechanism, we sometimes see ribosome-like densities in the center of the herniations

701 (Supplementary results figure 2C, rightmost panel). In maturation mode 2, a herniation curls over

702 another one, leading to an INM-INM inner bilayer. This is supported by the frequent observation of

703 clustered herniations in which multiple INM sheets are enclosed by a single ONM (Supplementary

704 results figure 2D). 
bioRxiv preprint doi: https://doi.org/10.1101/2022.03.04.483005; this version posted March 4, 2022. The copyright holder for this preprint (which was not certified by peer review) is the author/funder, who has granted bioRxiv a license to display the preprint in perpetuity. It is made available under aCC-BY-NC-ND 4.0 International license.
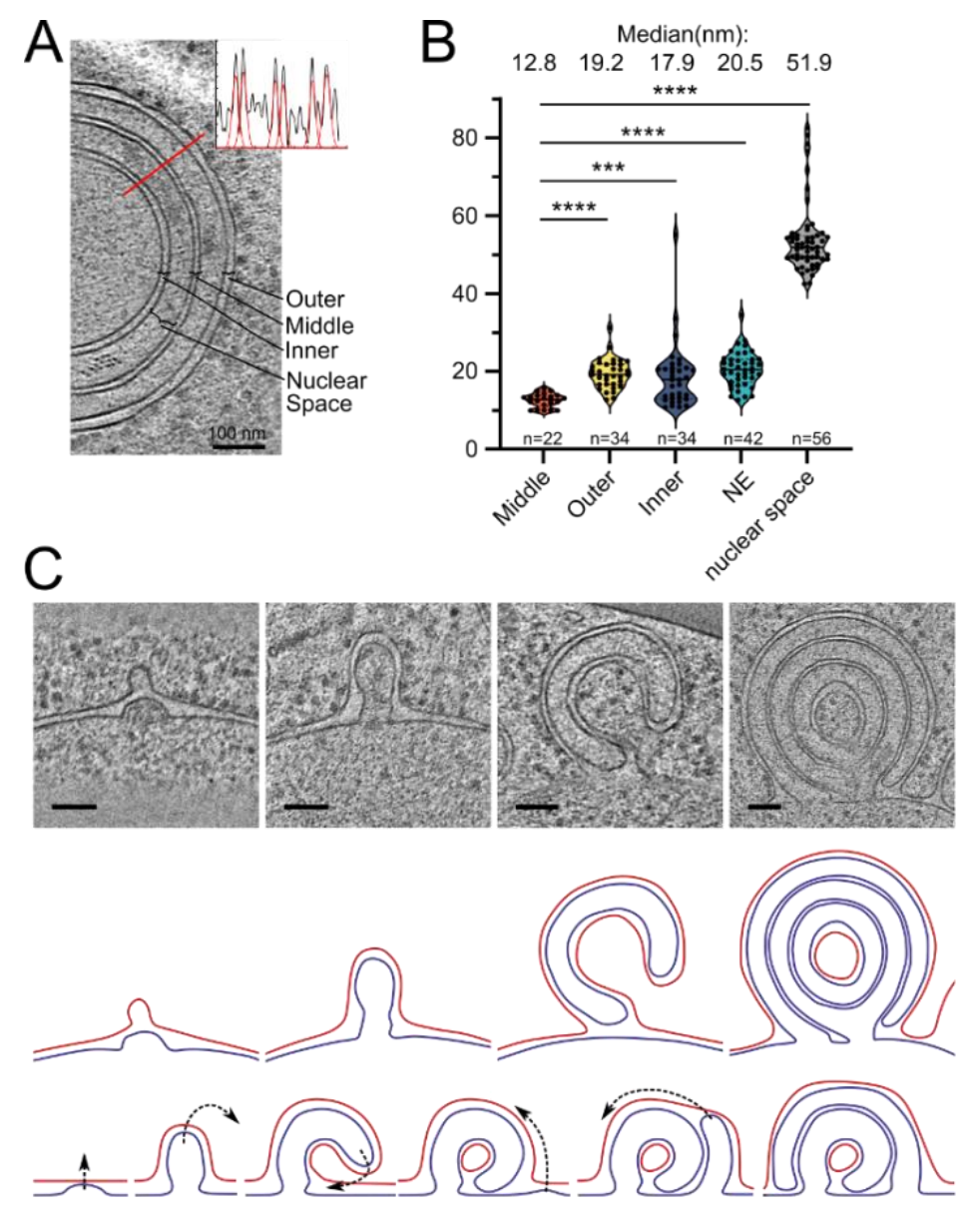

D
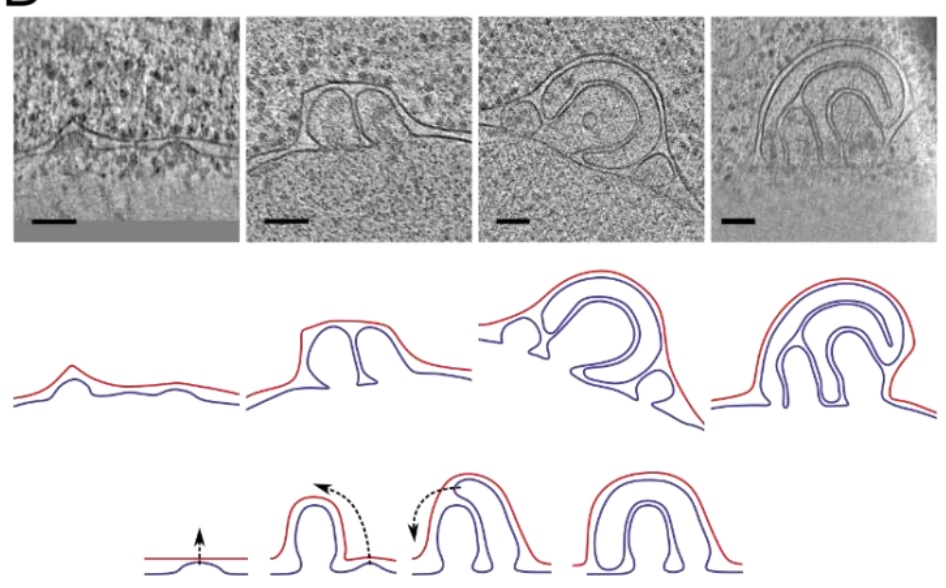

Supplementary results figure 2: Potential maturation processes of onion-like herniations. A) Tomographic slice of an onion-like herniation and an example line plot with fitted Gaussians measured at the indicated red line. Brackets indicate how the distances were classified for the plot in B). B) Violin plot with individual points of membrane-membrane distances. Mann-Whitney test, $* * * *$ : p-value <0.0001; $* * *$ : P-value $=0.0001$ C) Mode 1 for maturation of onion-like herniations. Top panel: tomographic slices of several stages of herniations in Br11(I395D) overexpressing cells (nucleus always in the bottom), middle panel: membrane segmentation of the

712 herniations of the upper panel. INM: blue, ONM: red; ONM in the center of the very right panel was classified as 
713 ONM based on the presence of ribosomes and wider membrane spacing. Lower panel: schematic of how the

714 onion-like herniations mature. D) Same as C) but for mode 2 of the maturation process of onion-like herniations.

715 Scale Bar: $100 \mathrm{~nm}$. Slice thickness: $1.4 \mathrm{~nm}$.

\section{Acknowledgements}

717 We are grateful to all the Weis lab members for many fruitful discussions and helpful suggestions. A.K

718 would like to thank A. C. Meinema for valuable discussions on and help with image analyses. We thank

719 P. Picotti and A. Leitner for the access to MS instruments, and the Center of Microscopy and Image

720 Analysis (ZMB), University of Zürich, and ScopeM, ETH Zürich, for microscopy support. We would

721 like to acknowledge the contributions of S. Steiger and J. Vailliant to early stages of this work during

722 their student projects. A.K. was a recipient of a Swiss National Science Foundation Marie Heim-

723 Voegtlin Fellowship (Grant number: PMPDP3_171317) and A.A.A. acknowledges the support from an

724 EMBO postdoctoral fellowship (Grant number: ALTF 910-2021). This study was supported by project

725 grants from the Swiss National Science Foundation (31003A_179275 to K.W and 31003A_179418 to

726 O.M.), a grant from the Research Council of Norway (NFR 315615 to E.O., K.W. and E.N.), and a grant from the German Research Foundation (DFG AN377/7-1 to W.A.)

\section{Competing interests}

729 The authors declare no conflict of interest.

\section{Supplementary material}

731 Movie S1 and S2: Sequential sections of a cryo-tomogram from Brl1 depleted cells. The tomogram is 6x binned; pixelsize: $2.1 \mathrm{~nm}$; scalebar: $100 \mathrm{~nm}$.

733 Movie S3 and S4: Sequential sections of a cryo-tomogram from non-depleted Brl1 control cells. The tomogram is 6x binned; pixelsize: $2.1 \mathrm{~nm}$; scalebar: $100 \mathrm{~nm}$.

735 Movie S5 and S6: Sequential sections of a cryo-tomogram from Brl1(I395D)-overexpressing cells. The 736 tomogram is 6x binned; pixelsize: $2.1 \mathrm{~nm}$; scalebar: $100 \mathrm{~nm}$.

737 Movie S7 and S8: Sequential sections of a cryo-tomogram from Brl1-overexpressing cells. The tomogram is $6 \mathrm{x}$ binned; pixelsize: $2.1 \mathrm{~nm}$; scalebar: $100 \mathrm{~nm}$. 


\section{Data availability}

740 The representative tomograms shown in the movies S1-S8 will be deposited in the EMDB under the accession number EMD-14503, EMD-14505 and EMD-14506. All raw MS data, the spectral libraries, the DIA data extractions generated with Spectronaut and the R code used for analysis will be uploaded in the PRIDE repository under the accession numbers PXD032017, PXD032016, PXD032024 and PXD032034 (Table 1). The proteomic data generated here was compared to a previously published dataset (Onischenko et al. 2020) available on PRIDE (PXD018034). The source data for the figures are provided. All blots presented in this study are provided in an uncropped format. Structure predictions for Brl1(P38770) and Brr6(P53062) can be accessed in the AlphaFold Protein Structure Database.

\section{Materials and methods}

\section{Plasmids and yeast strains construction}

750 Plasmids were generated according to standard molecular cloning techniques. The plasmids used in this study are listed in Supplementary File 1. Standard yeast genetic protocols were used for plasmid transformation and integration of linear DNA fragments into the yeast genome by homologous recombination. Strains used in this study are listed in Supplementary File 2. The heterozygous yeast strains BRL1/brl1 $1 a h$ (lacking amino acids 376 - 402) and BRL1/brll(I395D) were generated with CRISPR-Cas9 genome editing. Cloning details are available on request.

\section{Yeast culturing conditions}

757 Unless otherwise stated, yeast cultures were grown to mid $\log$ phase for at least $12 \mathrm{~h}$ at $30^{\circ} \mathrm{C}$. For 758 Western Blot analysis and fitness assays, cells were cultured in YPD medium (1\% yeast extract, 2\% 759 peptone, $2 \%$ dextrose) and for microscopy and proteomic analyses in synthetic complete medium (SCD,

$7606.7 \mathrm{~g} / \mathrm{L}$ yeast nitrogen base without amino acids, $2 \%$ dextrose) supplemented with the necessary amino 761 acids and nucleobases. Auxin-inducible degradation of Brl1 in log-phase yeast cultures with $\mathrm{OD}_{600}=$ $7620.1-0.2$ was induced by addition of IP6 ( $4 \mu \mathrm{M}$ phytic acid dipotassium salt, Sigma-Aldrich P5681) and either auxin (+auxin, $500 \mu \mathrm{M}$ indole-3-acetic acid in ethanol, Sigma-Aldrich I2886) or the equivalent amount of ethanol (-auxin) for the solvent control. Strains with galactose-inducible Brl1 constructs were pre-cultured in SC medium containing $2 \%$ raffinose. Expression was induced by supplementing $2 \%$ galactose to log-phase cultures $\mathrm{OD}_{600}=0.1-0.2$. For the metabolic labeling experiments, cells were initially grown in SCD containing light lysine (light $\mathrm{SCD}, 25 \mathrm{mg} / \mathrm{L}$ ) and then pulse labeled by medium exchange to SCD containing 13C6, 15N2 1-lysine (heavy SCD, Cambridge Isotope Laboratories, 25 $\mathrm{mg} / \mathrm{L}$ ). 


\section{$770 \quad$ Western blotting}

771 Auxin-inducible degradation was performed as described above (Yeast culturing conditions). At each post-degradation time point, an amount of cells corresponding to $2 \mathrm{OD}_{600}$ was collected by centrifugation and lysed by a $15 \mathrm{~min}$ incubation in $0.1 \mathrm{M}$ sodium hydroxide. Subsequently, cells were pelleted, resuspended in $50 \mu \mathrm{L}$ Laemmli sample buffer (10\% glycerol, 2\% SDS, 5\% 2-mercaptoethanol, $100 \mathrm{mM}$ DTT, $0.04 \%$ bromophenol blue, $62.5 \mathrm{mM}$ Tris-HCl pH6.8) and heat denatured for $5 \mathrm{~min}$ at $95^{\circ}$ C. Proteins were electrophoretically separated on an $8 \%$ polyacrylamide gel and then wettransferred to a nitrocellulose membrane (Amersham Protran 0.2 NC, GE Healthcare). Prior to antibody incubation, membranes were blocked for at least $2 \mathrm{~h}$ in 5\% PBST-milk (1 x PBS pH 7.4, 0.1\% Tween$20,5 \%$ dry milk). Then, membranes were incubated with primary antibody for $1 \mathrm{~h}$ at $\mathrm{RT}$, washed three times 10 min in PBST (1 x PBS pH 7.4; 0.1\% Tween-20) followed by 30 min incubation with secondary antibody at RT. Membranes were washed again three times for $10 \mathrm{~min}$ in PBST before fluorescence signal was imaged with the CLx ODYSSEY Li-COR. Primary antibodies used were mouse monoclonal $\alpha$-V5 (Invitrogen R960-25; 1:2'000) and rabbit monoclonal $\alpha$-hexokinase (US biologicals, H2035-01; 1:3,000). Secondary antibodies used were goat $\alpha$-mouse IgG Alexa Fluor 680 (Thermo Fisher Scientific, A-21057; 1:10,000), and goat $\alpha$-rabbit IgG IRDye800CW (Li-COR Biosciences, 926-32211; $1: 10,000)$.

For spot assays of strains overexpressing galactose-inducible Brl1 derivatives, strains were grown to saturation in SC medium supplemented with $2 \%$ raffinose and $0.1 \%$ glucose. Cells were plated on synthetic medium agar plates supplemented with $2 \%$ galactose in a five-fold serial dilution series starting with an $\mathrm{OD}_{600}$ of 1.0 using a 48-pin frogger. Strains derived from the nup1164GLFG $P_{M E T 3^{-}}$ NUP188 background were pre cultured in SCD lacking methionine and spotted on synthetic medium agar plates supplemented with or without methionine $(400 \mu \mathrm{g} / \mathrm{ml})$.

\section{Tetrad dissection}

795 Diploid yeast cells were grown on YPD for one day at $30{ }^{\circ} \mathrm{C}$ and then transferred to sporulation plates 796 (SPO; $1 \%$ potassium acetate, amino acids to $25 \%$ of normal concentration, $0.05 \%$ glucose, $2 \%$ agar) 797 and incubated for 5 days at RT. To digest the ascus wall, a pinhead-sized cell mass was incubated in 5 $798 \mu \mathrm{L}$ of Zymolyase $100 \mathrm{~T} 1 \mathrm{mg} / \mathrm{mL}$ (ICN, 320932) for $3 \mathrm{~min}$ at $30{ }^{\circ} \mathrm{C}$. Then, $300 \mu \mathrm{L}$ water was added to 799 stop the digestion, cells were shortly vortexed and spread on a YPD plate. Tetrads were dissected using

800 a Nikon Eclipse Ci-S dissecting scope and incubated for 2 days at $30^{\circ} \mathrm{C}$. Spore clones were tested for 801 genotype segregations by sequencing. 
802

803

804

805

806

807

808

809

810

811

812

813

814

815

816

817

818

819

820

821

822

823

824

825

826

827

828

829

830

831

832

833

834

\section{Fluorescence microscopy}

Cells were immobilized in a 384-well glass bottom plate (MatriPlate) coated with concanavalin A (Sigma-Aldrich). Imaging was performed with a 100x Plan-Apo VC objective (NA 1.4, Nikon) on a Nikon inverted epifluorescence Ti microscope equipped with a Spectra $\mathrm{X}$ LED light source (Lumencore) using the NIS Elements software (Nikon) at $30^{\circ} \mathrm{C}$ unless indicated differently. Images were acquired with a Flash 4.0 sCMOS camera (Hamamatsu) and processed using ImageJ software. Imaging of strains expressing the Nup170-RITE constructs was performed with a 100x Plan Apo lambda objective (NA 1.45oil DIC WD $0.13 \mathrm{~mm}$, Nikon) on a Nikon inverted Widefield Ti2-E microscope equipped with a Spectra III light engine and an Orca Fusion BT camera using the NIS Elements software (Nikon) at room temperature. Images were processed using the Denoise.ai and Clarify.ai algorithms from NIS Elements software and Fiji (Schindelin et al. 2012).

\section{Fluorescence recovery after photobleaching (FRAP)}

FRAP experiments were performed at room temperature on a Leica TCS SP8-AOBS microscope using a 63x 1.4NA Oil HC PL APO CS2 objective. Unidirectional scanner at speed of $1400 \mathrm{~Hz}$, NF488/561/633, an AU of 1.5 and a FRAP booster for bleaching were applied for every FRAP experiment using the PMT3 (500-551 nm) and PMT5 (575-694 nm) detectors. Image sizes of 512x75 at $80 \mathrm{~nm} / \mathrm{px}$ were used together with line accumulation of two, yielding a time interval of $120 \mathrm{~ms}$ per frame. 20 pre-bleach and 200 post-bleach frames were acquired. A $488 \mathrm{~nm}$ argon laser line was used at $20 \%$ base power in addition to a $561 \mathrm{~nm}$ DPSS laser line. Imaging was conducted with $1.5 \%$ laser intensity with a gain of 800 to illuminate the GFP, and $0.3 \%$ of the 561 laser power to illuminate mCherry. Bleaching was performed in a manually defined elliptical region comprising approximately one-third of the cell nucleus at $100 \%$ laser power of both laser lines for $120 \mathrm{~ms}$. For the case of mutant Brl1, the region was chosen to encompass part of a bright region (herniation). The mobility of GFPlabeled proteins in the bleached NE region was evaluated by quantifying the signal recovery in the bleached region. Extracellular background (Ibg) was subtracted from the intensity of the bleached region $\left(\mathrm{I}_{\mathrm{bl}}\right)$ and the values were bleach-corrected by normalizing for total cell intensity $\left(\mathrm{I}_{\text {total }}\right)$ resulting in $\left(\mathrm{I}_{\mathrm{b}-}-\mathrm{I}_{\mathrm{bg}}\right) /\left(\mathrm{I}_{\mathrm{total}}-\mathrm{I}_{\mathrm{bg}}\right)$ (Bancaud et al. 2010) using custom written scripts in MATLAB (Mathworks) and plotted with Prism 7 (GraphPad).

\section{Fluorescence microscopy of RITE constructs}

All strains expressing NUP-RITE constructs were grown to mid log phase in SCD supplemented with $300 \mu \mathrm{g} / \mathrm{mL}$ hygromycin B (Roche) to select for non-recombined cells. Prior to imaging, cells were centrifugally collected and recovered for $1 \mathrm{~h}$ in SCD without hygromycin B. Recombination was induced by addition of $\beta$-estradiol ( $1 \mu \mathrm{M}$ f.c., Sigma-Aldrich) and cells were imaged $3 \mathrm{~h}$ post induction. 
Strains expressing NUP170-RITE constructs were grown to mid log-phase in SD -URA to select for non-recombined cells. Prior to imaging, recombination was induced by addition of $\beta$-estradiol $(1 \mu \mathrm{M}$ f.c., Sigma-Aldrich) and uracil and cells were imaged $\sim 30$ min (new Nup170-RITE) or $\sim 5$ h (old Nup170-RITE) post induction.

\section{Quantitative image analysis}

We used the automated imaging analysis pipeline NuRim to quantify the fluorescence intensity signal in the nuclear envelope for various NUP GFP fusion proteins (Rajoo et al. 2018; Vallotton et al. 2019). In brief, nuclear contours were called in an unbiased manner based on the fiducial marker dsREDHDEL. Fluorescence intensities of NUP-yEGFP along these contours were then extracted in ImageJ. NE intensity profiles with large foci in the NE were excluded by using an intensity value standard variation cutoff of 200, in Brl1-depleted cells this accounted for maximum 35\% of the generated masks. Brightness and contrast of the presented images were adjusted the same for all images in one panel unless otherwise indicated using Fiji. Graphical representation of the data was carried out in R.

For the colocalization plots (Figure 6D \& Figure S5B) at least 36 line plots (exact number indicated in respective figures) were manually generated in Fiji. Values for each line plot were centered according to the peak intensity of the Brl1(I395D)-mcherry signal and plotted as mean with SD. Graphs were created with Prism 9.

In strains expressing NUP170-RITE fusion proteins, the NE contours were manually delineated based on the Brl1-mCherry signal and the intensity profiles obtained using Fiji. Pearson's correlation coefficient between intensity values in green and red channels were calculated. Only cells with foci in both red and green channels were selected for quantification. The following cells were excluded: NE contours with no signals in any of the two channels, cells with a strong red background signal, cells that did not undergo recombination.

\section{Recombinant protein expression and purification}

The fusion proteins 6xHis-MBP-TEV-yEGFP, 6xHis-MBP-TEV-ahBrl1-yEGFP, 6xHis-MBP-TEVahBrl1- (F391D)-yEGFP and 6xHis-MBP-TEV-ahBrl1(I395D)-yEGFP were expressed in E.coli BL21 RIL cells. Bacteria were cultured in 1 L YT (0.8\% Bacto-tryptone, 0.5\% Bacto-yeast extract, $86 \mathrm{mM}$ sodium chloride) to OD600 $=0.8-1.0$ at $37^{\circ} \mathrm{C}$, and protein expression was induced by adding $0.2 \mathrm{mM}$ IPTG (AppliChem A1008,0025) and cells were grown ON. The next day cells were harvested in a AF6.100 rotor (Herolab) for 15 minutes at 5'000 RPM at $4{ }^{\circ} \mathrm{C}$. Pellets were resuspended in $20 \mathrm{ml}$ Tris$\mathrm{HCl}(20 \mathrm{mM}, \mathrm{pH} 7.5)$ supplemented with $10 \mathrm{ug} / \mathrm{mL}$ DNase I (Roche, 10104159001) and $1 / 2$ tablet cOmplete Protease Inhibitor Cocktail (Sigma-Aldrich, 05053489001). Cells were lysed using the Avestin Emulsiflex c5 (ATA Scientific) and centrifuged at $4^{\circ} \mathrm{C}$ for 15 minutes at 12'000 RPM in the SS-34 rotor (Thermo Scientific). Supernatant was filtered through a $0.45 \mu \mathrm{m}$ filter, applied to $\sim 1 \mathrm{ml} \mathrm{Ni-}$ 
NTA Agarose (Qiagen 30210), and incubated for $1 \mathrm{~h}$ at $4^{\circ} \mathrm{C}$. The agarose was washed thoroughly with $20 \mathrm{mM}$ Tris- $\mathrm{HCl} \mathrm{pH} 7.5,500 \mathrm{mM} \mathrm{NaCl}, 30 \mathrm{mM}$ Imidazole prior to elution with $20 \mathrm{mM}$ TrisHCl $\mathrm{pH} 7.5$, $500 \mathrm{mM} \mathrm{NaCl}, 400 \mathrm{mM}$ Imidazole. Purified proteins were dialysed $\mathrm{ON}$ in $20 \mathrm{mM}$ Tris $\mathrm{pH}$ 7.5, $150 \mathrm{mM}$ $\mathrm{NaCl}$ at $4^{\circ} \mathrm{C}$, concentrated in $1 \mathrm{~mL}$ in a Vivaspin Turbo 4 (30’000 MWCO, Sartorius VS04T22) and further purified on a Superdex 75 10/300 gel filtration column (GE Healthcare).

\section{Liposome binding assay}

Liposome generation and flotation was performed as described in Vollmer et al. (Vollmer et al. 2015). In short, E. coli polar lipids (Avanti polar lipids) dissolved in chloroform and supplemented with 0.2 mol \% 18:1 Liss Rhodamine PE (Avanti polar lipids) were vacuum dried on a rotary evaporator, dissolved as liposomes in PBS by freeze/thawing cycles and extruded by passages through Nuclepore Track-Etched Membranes (Whatman) with defined pore sizes using an Avanti Mini-Extruder to generate small unilamellar liposomes of defined sizes. For liposome flotations proteins $(6 \mu \mathrm{M})$ were mixed 1:1 with liposomes $(6 \mathrm{mg} / \mathrm{ml})$ and floated for $2 \mathrm{~h}$ at $55000 \mathrm{rpm}$ in a TLS-55 rotor (Beckman) at $25^{\circ} \mathrm{C}$ through a sucrose gradient. Binding efficiency was determined by Western Blot analysis using an EGFP-antibody (Roche 11814460001) and the ImageQuant LAS-4000 system (Fuji) and the AIDA software, comparing band intensities of start materials with floated liposome fraction.

\section{Sequence alignment}

Sequence alignment was performed using the COBALT web server (https://www.ncbi.nlm.nih.gov/tools/cobalt/re_cobalt.cgi) and visualized using Jalview (Waterhouse et al. 2009).

\section{Cryo-FIB milling of yeast cells}

Brl1 of exponentially growing yeast cells was inducibly depleted as described above. As control for the Brl1 degradation, cells lacking OSTIR1 were treated for 4-4.5 h with auxin. Br11(I395D) overexpressing cells were grown as described above and as a control, cells overexpressing Brl1 were cultured for $6 \mathrm{~h}$ in SC 2\% galactose. Cells were pipetted onto Quantifoil Cu R2/1 grids (Quantifoil), blotted for 4 s and plunge frozen using a manual plunger. Blotting was performed manually from the backside of the grid. Cryo FIB-milling was performed essentially as previously described (Wagner et al. 2020). In brief, the grids were transferred to a Leica BAF060 system equipped with a Leica cryo transfer system at $160^{\circ} \mathrm{C}$ and grids were coated with $\sim 5 \mathrm{~nm} \mathrm{Pt} / \mathrm{C}$. Afterwards grids were transferred to a Zeiss Auriga 40 Crossbeam FIB-SEM equipped with cryostage and cryo-transfer shuttle. An organometallic platinum layer was deposited using the integrated gas injection system. Cells were milled in three steps at $30 \mathrm{kV}$ using rectangle patterns ( $240 \mathrm{pA}$ to $\sim 200 \mu \mathrm{m}, 120 \mathrm{pA}$ to $\sim 100 \mu \mathrm{m}, 50 / 30 \mathrm{pA}$ to $<0.3 \mu \mathrm{m}$ ) to a target thickness of $<250 \mathrm{~nm}$ and samples were stored in liquid nitrogen until data acquisition. 


\section{Cryo Electron tomography}

903 Tilt series of FIB-milled lamella were acquired using a Titan Krios equipped with a Gatan Quantum 904 Energy Filter and a K2 Summit electron detector or a Titan Krios G3i equipped with a Gatan 905 BioQuantum Energy Filter and K3 direct electron detector at $300 \mathrm{kV}$. Tilt series were acquired using 906 SerialEM (Mastronarde 2003) at a pixel size of $3.4 \AA$ at the specimen level. The target defocus was set 907 to -4 to $-7 \mu \mathrm{m}$ and tilt series were acquired using a dose symmetric tilt scheme (Hagen, Wan, and Briggs 908 2017) from $-65^{\circ}$ to $55^{\circ}$ with an increment of $3^{\circ}$ and a total dose of $\sim 140$ electrons per angstrom squared.

\section{Tomogram reconstruction}

910 Movie frames were aligned using IMODs alignframes function (Mastronarde and Held 2017). Tilt series 911 were processed and aligned using the IMOD suite. Alignment was performed using the $4 \mathrm{x}$ binned 912 projections and the patch tracking function in IMOD. Outliers in patch tracking (e.g., patch aligning on 913 ice contamination) were manually corrected. Occasionally, contaminations on top of the lamella were 914 used as fiducial markers. Overview tomograms for particle picking were reconstructed using the SIRT915 like filter with $12 \mathrm{x}$ iterations and $4 \mathrm{x}$ binning. NPCs and NPC-herniations coordinates and rough 916 orientation along the nuclear envelope were picked and determined manually.

\section{Quantification of herniations and NPCs}

918 For the quantification of herniations and NPCs in Brl1 depleted cells we used 51 tomograms. For this 919 analysis we also included tomograms with lower quality which we did not include in the subtomogram 920 analysis described below. For the control condition we used 27 tomograms of cells subjected to the 921 same treatment but without OsTIR1 plasmid. For the quantification of herniations and NPCs in 922 Brl1(I395D) overexpressing cells, 50 tomograms were analyzed. For our control condition in cells 923 overexpressing Brl1 without the point mutation we used 17 tomograms. To compensate for the different 924 surface area of NE in tomograms, we normalized the number of NPCs and herniations by the area of 925 NE in each tomogram. For this, we manually segmented the NE in three tomographic slices using the 926 drawing tool in IMOD. Segmentations for all other slices were interpolated. We then calculated the 927 distance between segmentation points to determine the total visible surface area in MATLAB and used 928 Prism 9 (GraphPad) for visualization.

\section{Subtomogram averaging}

930 Subtomograms containing NPCs or herniations were reconstructed in IMOD from unbinned, dose931 filtered and CTF-corrected tilt series. CTF was corrected as described previously by estimating the mean defocus by strip-based periodogram averaging. With the information for the mean defocus, the

933 tilt angle and axis orientation, the defocus gradient for each projection was calculated and according to 934 the defocus gradient, each projection was CTF-corrected by phase flipping (Eibauer et al. 2015). CTF- 
corrected stacks were dose-filtered using the IMOD mtffilter function and subtomograms reconstructed using IMOD.

We reconstructed 85 herniation-containing subtomograms from 31 tomograms of Brl1-depleted cells. Based on the curvature of the ONM, herniations were classified manually into INM-evaginations ( $\mathrm{n}=25$ ) and herniations $(n=60)$. When the ONM was not or only slightly deformed we classified the herniation as an INM-evagination (examples in figure S2D). As a control we reconstructed 29 mature NPC from 19 tomograms of the same dataset. For Brl1(I395D) overexpressing cells, we reconstructed 47 herniations from 21 tomograms.

Prealigned full NPCs/herniations were aligned using iterative missing wedge weighted subtomogram alignment and averaging using the TOM toolbox (Friedrich et al. 2005; Nickell et al. 2005), by merging the half set averages after each iteration as a template for the next iteration. 8x binned subtomograms were aligned using 8-fold rotational symmetry. For averaging mature NPCs and Brl1(I395D) herniations we further extracted 8 protomers (4x binned) according to the 8 fold symmetry of the NPC. Protomers outside the lamella were excluded by manual inspection. For mature NPCs we used 179 protomers (53 excluded from 232 protomers) for the final average. For Brl1(I395D) herniations we used 237 protomers (139 excluded from 376 protomers) for the final average.

For the different forms of herniations in Brl1 depleted conditions, protomer alignment did not improve the maps. We think that resolution of these averages is limited because of the high heterogeneity of herniations in overall shape and membrane curvature. We also believe that the electron-dense center of herniations in Brl1-depleted cells limited the resolution of our average. Several trials with different masks, bandpass filters and classification based on membrane curvature did not improve resolution. Further, our subtomogram average of herniations in Brl1(I395D) overexpressing cells, which do not have an electron-dense center, shows distinct IR-like densities, and is better resolved although less subtomograms were used.

Resolution was determined using masked half maps and the webserver https://www.ebi.ac.uk/emdb/validation/fsc. Final maps were filtered according to the achieved resolution at FSC 0.5 (INM-evaginations: $12 \mathrm{~nm}$, herniations (Brl1-AID): $11 \mathrm{~nm}$, Brl1(I395D) herniation: $8 \mathrm{~nm}$, mature NPC: $8 \mathrm{~nm}$ ). The full-pore map for the mature NPC and the Brl1(I395D) herniations were stitched from single protomers by fitting the protomer-average into the full-NPC map in UCSF Chimera (Pettersen et al. 2004).

\section{AlphaFold prediction}

To predict the structure of Brl1 we used the python script for AlphaFold2.1.1 (Jumper et al. 2021) implemented in SBGrid with standard settings and the mode_preset=monomer_ptm setting. Since we locally predicted the structure of Brl1, it is not identical to the structure in the AlphaFold database. However, the structured part is almost identical (rmsd: $1.35 \AA$ ) and only the unstructured $\mathrm{N}$-and C- 
termini deviate between the structures significantly. Visualization of prediction metrics were generated using the following jupyter notebook in Anaconda:

\section{https://colab.research.google.com/drive/1CizC7zmYvFkav5qfBbWxhgUHrOxwym2w).}

\section{Dimension-measurements on onion-like herniations in Brl1(I395D) overexpressing cells} 4x binned tomograms of Br11(I395D) overexpressing cells were processed in Fiji using a Gaussian blur with a sigma of 1 and contrast was inverted. Per onion-like herniation, 3-4 line plots were generated and exported to MATLAB. Peaks (=membranes) of the line plots were determined by Gaussian fit of the peaks. 11 onion-like herniation from 8 tomograms were analyzed. The same procedure was performed on the NE of tomograms of Brl1 overexpressing cells. 6-9 line plots per NE were generated and $5 \mathrm{NE}$ from 5 tomograms were analyzed. Only tomograms where the herniation or the NE were roughly perpendicular in the section were used. Visualization and statistical tests performed in Prism 9.

\section{Visualization of tomograms and subtomograms}

Snapshots of single NPCs or herniations were extracted from $4 \mathrm{x}$ binned tomograms reconstructed in IMOD using the SIRT like filter with 12 iterations and visualized using tom_volxyz (Figure S2D and 7C). All tomographic slices shown were reconstructed using IMOD's SIRT like filter with 12 iterations and slice thickness is indicated in figure legends.

All procedures were implemented in MATLAB and using the TOM toolbox. Chimera, IMOD and Alphafold were used as part of SBGrid (Morin et al. 2013).

\section{Preparation of IgG-Coupled Dynabeads}

IgG-coupled Dynabeads were prepared as described in Alber et al (Alber et al. 2007). $150 \mathrm{mg}$ of magnetic Dynabeads were resuspended in $9 \mathrm{~mL}$ fresh $0.1 \mathrm{M}$ sodium phosphate buffer $(22.5 \mathrm{mM}$ monosodium phosphate, $81 \mathrm{mM}$ disodium phosphate, $\mathrm{pH}$ 7.4). Bead suspension was vortexed for $30 \mathrm{~s}$ followed by a $10 \mathrm{~min}$ incubation at room temperature under constant agitation. Then, beads were placed onto a magnetic holder, clear buffer was aspirated off and beads were washed once with $4 \mathrm{~mL} 0.1 \mathrm{M}$ sodium phosphate buffer. Antibody mix was prepared by resuspending $50 \mathrm{mG}$ rabbit IgG powder in 2.1 $\mathrm{mL}$ distilled water and spinning down the mixture for $10 \mathrm{~min}$ at $15^{\prime} 000 \mathrm{~g}$ in a tabletop centrifuge precooled to $4^{\circ} \mathrm{C}$. Clear supernatant was transferred to a fresh falcon tube and $4.275 \mathrm{~mL} 0.1 \mathrm{M}$ sodium phosphate buffer was added. To this, $3 \mathrm{M}$ ammonium sulfate buffer (3 $\mathrm{M}$ ammonium sulfate dissolved in $0.1 \mathrm{M}$ sodium phosphate buffer) was added slowly, constantly shaking the mixture. The antibody mix was then filtered through a $22 \mu \mathrm{m}$ Millex GP filter and was ready for use. The magnetic Dynabeads were incubated with the antibody mix for $\sim 20 \mathrm{~h}$ on a rotating wheel at $30^{\circ} \mathrm{C}$. Thereafter, beads were briefly washed once with $100 \mathrm{mM}$ glycine $\mathrm{HCl} \mathrm{pH} 2.5,10 \mathrm{mM}$ Tris- $\mathrm{HCl} \mathrm{pH} 8.8$ and $100 \mathrm{mM}$ freshly 
1003

1004

1005

1006

1007

1008

1009

1010

1011

1012

1013

1014

1015

1016

1017

1018

1019

1020

1021

1022

1023

1024

1025

1026

1027

1028

1029

1030

1031

1032

1033

1034

1035

1036

prepared triethylamine. This was followed by four 5 min washes with PBS pH 7.4 and two $10 \mathrm{~min}$ washes with PBS pH 7.4 containing $0.5 \%$ Triton X-100. Beads were finally resuspended in a total of 1 mL PBS supplemented with $0.02 \%$ sodium azide resulting in a concentration of $100 \mathrm{mg}$ beads $/ \mathrm{mL}$ and stored at $4^{\circ} \mathrm{C}$

\section{Metabolic labeling assays}

Yeast strains harboring endogenously tagged Brl1-ZZ or Nup170-ZZ fusion proteins were cultured for a minimum of $16 \mathrm{~h}$ at $30^{\circ} \mathrm{C}$ in light $\mathrm{SCD}$. Cell culture samples equivalent to $250 \mathrm{~mL} \mathrm{OD} 600=1.0$ were collected by filtration on an $0.8 \mu \mathrm{L}$ nitrocellulose membrane. During harvesting, the cells were briefly washed twice with $25 \mathrm{~mL}$ distilled water directly on the filter membrane and then snap-frozen in liquid nitrogen. Samples corresponding to the $0 \mathrm{~h}$ timepoint were collected immediately before labeling onset. Thereafter, cell cultures were pulse labeled as follows, the amount of log-phase cell cultures corresponding to $650 \mathrm{~mL}$ of $\mathrm{OD}_{600}=1.0$ were washed them on the filter with $50 \mathrm{~mL}$ heavy $\mathrm{SCD}$ containing 13C6, 15N2 1-lysine ( $25 \mathrm{mg} / \mathrm{L}$, Cambridge Isotope Laboratories) and reinoculated in heavy SCD. For the experiments with the Brl1-AID constructs, cultures were split in half and switched to heavy SCD containing IP6 (4 $\mu \mathrm{M}$ f.c.) and either auxin (500 $\mu \mathrm{M}$ f.c.) or the equivalent volume of ethanol for the solvent control. Post labeling timepoints were collected in regular intervals as described above. During the time course all cultures were maintained in logarithmic growth by periodic dilution with the respective prewarmed medium.

\section{Affinity pulldowns}

All the following procedures were performed under ice cold conditions. Frozen yeast pellets were resuspended in $1 \mathrm{~mL}$ Lysis Buffer (20 mM HEPES pH 7.5, $50 \mathrm{mM}$ KOAc, $20 \mathrm{mM} \mathrm{NaCl}, 2 \mathrm{mM} \mathrm{MgCl}$, $1 \mathrm{mM}$ DTT, 10\% v/v glycerol) and transferred into $2 \mathrm{ml} \mathrm{screw-cap} \mathrm{micro} \mathrm{tubes} \mathrm{(Sarstedt} \mathrm{Inc)} \mathrm{pre-filled}$ with approximately $1 \mathrm{ml}$ of $0.5 \mathrm{~mm}$ glass beads (Biospec products). Cell material was spun down in a tabletop centrifuge and the tubes were filled up completely with Lysis Buffer. During this step, extra care was taken to avoid any air inclusion. Cells were mechanically lysed with a mini BeadBeater-24 (BioSpec Products) in four 1 min cycles at 3500 oscillations per minute with 1 min cooling intermissions in ice-water. Cell lysates were then spun down for $30 \mathrm{~s}$ at $15^{\prime} 000 \mathrm{~g}$ in a table-top centrifuge precooled to $4{ }^{\circ} \mathrm{C}$. $150 \mu \mathrm{L}$ of the supernatant was frozen in liquid nitrogen for the analysis of the source cell lysates. For the affinity pulldowns, $1 \mathrm{~mL}$ of the supernatant was supplemented with $110 \mathrm{~mL} 10 \mathrm{x}$ Detergent mix (protease inhibitor cocktail (Sigma-Aldrich), 5\% v/v Triton x-100, 1\% v/v Tween-20 in Lysis Buffer) and $2 \mathrm{mg}$ IgG Dynabeads, pre-equilibrated two times with Equilibration Buffer (0.5\% v/v Triton X-100 and 0.1\% v/v Tween-20 in Lysis Buffer). The remaining supernatant was frozen in liquid nitrogen for the analysis of the source cell lysates. Following a 30 min incubation of the affinity pulldown samples at $4^{\circ} \mathrm{C}$ under constant agitation, the beads were washed twice with 1 

ml Wash Buffer (0.1\% v/v Tween-20 in Lysis Buffer). Proteins were eluted in $40 \mu \mathrm{L}$ 1x Laemmli sample buffer for $2 \mathrm{~min}$ at $50^{\circ} \mathrm{C}$. Finally, elutes were completely denatured at $95^{\circ} \mathrm{C}$ for $5 \mathrm{~min}$ and frozen in liquid nitrogen.

\section{In-gel tryptic digestion}

Eluted proteins were electrophoretically concentrated by SDS-PAGE in a 4\% acrylamide stacking gel. Proteins were visualized by incubation with Coomassie SimplyBlue SafeStain (Invitrogen), followed by destaining for at least $14 \mathrm{~h}$ in distilled water. Protein bands were cut out and processed according to a standard in-gel digestion protocol. In brief, disulfide bonds were reduced with dithiothreitol $(6.5 \mathrm{mM}$ DTT in $100 \mathrm{mM}$ ammonium bicarbonate) for $1 \mathrm{~h}$ at $60^{\circ} \mathrm{C}$, proteins were alkylated with iodoacetamide (54 $\mathrm{mM}$ in $100 \mathrm{mM}$ ammonium bicarbonate) for $30 \mathrm{~min}$ at $30^{\circ} \mathrm{C}$ in the dark and finally tryptically digested with $1.25 \mu \mathrm{g}$ of sequencing grade porcine trypsin (Promega) in $100 \mathrm{mM}$ ammonium bicarbonate at $37^{\circ} \mathrm{C}$ for $16 \mathrm{~h}$. The resulting peptides were loaded in pre-equilibrated C18 BioPureSPN mini columns (The Nest Group, Inc.), washed and desalted 3 times with Buffer A ( $0.1 \%$ formic acid in HPLC-grade water), eluted three times with $50 \mu \mathrm{L}$ Buffer B (50\% acetonitrile, $0.1 \%$ formic acid in HPLC-grade water) and finally recovered in $12.5 \mu \mathrm{L}$ Buffer A supplemented with iRT peptides (1:50 v:v, Biognosys).

\section{Tryptic digestion of source cell lysates}

The source lysates of Brl1 APs 90 min post labeling were adjusted to $50 \mu \mathrm{L}$ with a protein concentration of $4 \mu \mathrm{g} / \mu \mathrm{L}$ with lysis buffer as determined by the Bradford method (Bio-Rad). Samples were diluted with $200 \mu \mathrm{L}$ guanidine chloride (7 M in $100 \mathrm{mM}$ ammonium bicarbonate) to reach a final guanidine chloride concentration of $5.6 \mathrm{M}$. Disulfide bonds were reduced with DTT ( $6.5 \mathrm{mM}$ f.c.) at $37^{\circ} \mathrm{C}$ for 45 min and alkylated with iodoacetamide supplemented to $54 \mathrm{mM}$ f.c. at $30^{\circ} \mathrm{C}$ in the dark for $30 \mathrm{~min}$. The samples were then diluted to a final guanidine chloride concentration of $1 \mathrm{M}$ with $100 \mathrm{mM}$ ammonium bicarbonate and digested with sequencing grade porcine trypsin (Promega, 1:100 trypsin:protein) for $22 \mathrm{~h}$ at $37^{\circ} \mathrm{C}$. Digestion was quenched by addition of $3 \%(\mathrm{v} / \mathrm{v})$ of $100 \%$ formic acid ( $\mathrm{pH} \sim 2.0$ ) and peptides were desalted in a BioPureSPN MACRO spin columns (The Nest Group, Inc.) as described above (Tryptic in-gel digestion). Tryptic peptides were diluted to $1 \mu \mathrm{g} / \mu \mathrm{L}$ with Buffer A based on $\mathrm{OD}_{280}$ readouts and the samples were spiked with 1:50 (v:v) iRT peptides (Escher et al. 2012) for the mass spectrometry acquisition.

\section{Lysate intermixing tests}

For the lysate intermixing tests $200 \mathrm{OD}_{600}$ of an untagged cell culture grown in heavy medium was mixed with the equivalent amount of cell culture expressing an affinity tagged protein and grown in light medium. The mixture was subjected to the affinity isolation procedure and processed for mass 
1070

1071

1072

1073

1074

1075

1076

1077

1078

1079

1080

1081

1082

1083

1084

1085

1086

1087

1088

1089

1090

1091

1092

1093

1094

1095

1096

1097

1098

1099

1100

1101

1102

1103

spectrometric analysis as described above. For Brl1-AID strains, the depletion was induced $5 \mathrm{~h}$ prior to harvesting by addition of IP6 (4 $\mu \mathrm{M}$ f.c.) and either auxin (500 $\mu \mathrm{M}$ f.c.) or ethanol for the solvent control.

\section{DDA MS assays}

Unlabeled Brl1 AP samples were assayed in a data-dependent acquisition mode (DDA), for subsequent spectral library generation (see "DIA MS data extraction"). LC-MS/MS analysis was performed on an Orbitrap Fusion Lumos Tribrid mass spectrometer (Thermo Scientific) coupled to an EASY-nLC 1200 system (Thermo Scientific). Peptides were separated on an Acclaim PepMap 100 C18 (25 cm length, $75 \mu \mathrm{m}$ inner diameter) with a two-step linear gradient from 5\% to $30 \%$ acetonitrile in 120 minutes and from $30 \%$ to $40 \%$ acetonitrile in 10 minutes at a flow rate of $300 \mathrm{nl} / \mathrm{min}$. The DDA acquisition mode was set to perform one MS1 scan followed by MS2 scans for a cycle time of $3 \mathrm{~s}$. The MS1 scan was performed in the Orbitrap ( $\mathrm{R}=120^{\prime} 000,100^{\prime} 000$ AGC target, maximum injection time of $100 \mathrm{~ms}$ and scan range $350-1400 \mathrm{~m} / \mathrm{z}$ ). Peptides with charge state between 2-7 were selected for fragmentation (isolation window: $1.6 \mathrm{~m} / \mathrm{z}$ and fragmentation with $\mathrm{HCD}$, NCE 28\%) and MS2 scans were acquired in a Orbitrap $\left(\mathrm{R}=30^{\prime} 000,100^{\prime} 000\right.$ AGC target, maximum injection time of $\left.54 \mathrm{~ms}\right)$. A dynamic exclusion of $30 \mathrm{~s}$ was applied.

\section{DIA MS assays}

Data independent acquisition (DIA) assays were performed on two different instrument setups (Orbitrap Fusion Lumos Tribrid (DIA:A) for the Brl1 AP samples and Orbitrap QExactive+ (DIA:B) for the Nup170 AP samples and the lysis intermixing assays).

DIA:A. LC-MS/MS analysis was performed on an Orbitrap Fusion Lumos Tribrid mass spectrometer (Thermo Scientific) coupled to an EASY-nLC 1200 system (Thermo Scientific). Peptides were separated as described in "DDA MS assays". DIA acquisition was performed with the following parameters: one MS1 scan (350-2000 m/z) with variable windows from 350 to $1150 \mathrm{~m} / \mathrm{z}$ with $1 \mathrm{~m} / \mathrm{z}$ overlap for a cycle time of $3 \mathrm{~s}$. Ions were fragmented with HCD (NCE 28\%). The MS1 scan was performed at 120'000 R, 200'000 AGC target and 100 ms injection time, the MS2 scan at 30'000 R, 500 '000 AGC target and 54 ms injection time.

DIA:B. LC-MS/MS was performed on an Orbitrap QExactive+ mass spectrometer (Thermo Fisher) coupled to an EASY-nLC-1000 liquid chromatography system (Thermo Fisher). Peptides were separated using a reverse phase column (75 $\mu \mathrm{m}$ ID x $400 \mathrm{~mm}$ New Objective, in-house packed with ReproSil Gold 120 C18, $1.9 \mu$ m, Dr. Maisch GmbH) across a two-step linear gradient: from 3\% to 25\% acetonitrile in $160 \mathrm{~min}$ and from $24 \%$ to $40 \%$ in $20 \mathrm{~min}$ at a flow rate of $300 \mathrm{nl} / \mathrm{min}$. DIA acquisition was performed with the following parameters: one MS1 scan $(350-1500 \mathrm{~m} / \mathrm{z})$ with 20 variable windows from 350 to $1400 \mathrm{~m} / \mathrm{z}$ with 1m/z overlap. Ions were fragmented with HCD (NCE 25\%). The MS1 scan 

R, 1'000'000 AGC target and auto injection time.

\section{PRM MS assays}

1107 Parallel reaction monitoring (PRM) assays were performed with the two different instrument setups 1108 described in "DIA MS assays" (Orbitrap Fusion Lumos Tribrid (PRM:A) and Orbitrap QExactive+ 1109 (PRM:B)).

1110 PRM:A. Peptides were separated as described in "DIA MS assays - DIA:A”. MS analysis of the targeted 1111 peptides was set up with the combination of one untargeted MS1 scan (120'000 R, 200'000 AGC

1112 Target, injection time $100 \mathrm{~ms}$ ) followed by 106 scheduled targeted scans (AGC $=450$ '000, resolution 1113 and injection time was variable based on peptide response) using an isolation window of $1.8 \mathrm{~m} / \mathrm{z}$ and 1114 HCD fragmentation $(\mathrm{NCE}=28 \%)$.

1115 PRM:B. Peptides were separated using a reverse phase column (75 $\mu \mathrm{m}$ ID x $400 \mathrm{~mm}$ New Objective, 1116 in-house packed with ReproSil Gold 120 C18, $1.9 \mu \mathrm{m}$, Dr. Maisch GmbH) across a linear gradient from $11175 \%$ to $40 \%$ acetonitrile in $90 \mathrm{~min}$. MS acquisition of the targeted peptide was set up with the 1118 combination of one untargeted MS1 scan (70’000 R, 3‘000’000 AGC Target, injection time $100 \mathrm{~ms}$ ) 1119 followed by 55 scheduled targeted scan ( $\mathrm{AGC}=1^{`} 050$ ’000, resolution 35’000 and $110 \mathrm{~ms}$ injection 1120 time) using an isolation window of $1.8 \mathrm{~m} / \mathrm{z}$ and $\mathrm{HCD}$ fragmentation $(\mathrm{NCE}=27 \%)$

\section{$1121 \quad$ PRM data analysis}

1122 The metabolic labeling of proteins in the source cell lysates was analyzed by parallel reaction 1123 monitoring MS (PRM) 90 min after the pulse labeling onset. Probed proteins included NUPs that 1124 exhibited outstandingly high or low labeling kinetics in the Brl1 AP, two NTRs (Kap123 and Mex67) 1125 and two randomly picked co-purified proteins (Rrp5 and Acc1). Precursors for the targeted analysis 1126 were selected based on good labeling consistency with other peptides of the same protein, high intensity and low number of missing values in the Brl1 APs. Peptides with missed cleavage sites or with cysteine and methionine residues were excluded when possible. All proteins were represented by 2-5 peptides.

1129 Targeted data analysis was performed as described in "PRM MS assays" and resulting intensities were 1130 analyzed with Skyline daily (64 bit, 20.1.1.213 version). Precursor ions identified by at least 3-4 1131 coeluting light and heavy transitions were quantified by manual peak integration. For precursor ions 1132 that were well detected in both heavy and light channels the respective intensities were calculated as 1133 the sum of the top3 most intense transitions in each channel. Fractional protein labeling was quantified 1134 as $\mathrm{H} /(\mathrm{H}+\mathrm{L})$, where $\mathrm{H}$ and $\mathrm{L}$ are the summed intensities of the above protein-born precursors in heavy and light channels, respectively. 


\section{DIA MS data extraction}

1137 Two Hybrid spectral libraries were generated with Spectronaut v.15 (Biognosys AG) using the 1138 combination of 20 DDA and 30 DIA datasets originating from APs with 10 NUP baits (Onischenko et 1139 al. 2020), and 4 DIA and 6 DDA datasets from Brl1 and Nup170 bait APs acquired in this study. The 1140 label-free assay library contained $\mathrm{b}$ and y transition ions (for a total of 3'918 protein groups, 75'780 1141 precursors and 105'089 transitions). The SILAC assay library comprised y transitions only, with the 1142 heavy-channel $(\mathrm{K}+8.014199)$ generated in silico using the "inverted spike in" workflow (for a total of 1143 3'825 protein groups, 97'069 precursors). Only tryptic peptides with a maximum of two missed 1144 cleavages were considered. Carbamidomethylation was set as fixed modification and methionine oxidation was set as variable modification. Spectra were searched against the SGD protein database 1146 (downloaded on 13.10.2015, 6'713 entries) concatenated with entries for contaminants and iRT 1147 peptides using a 1\% FDR control at peptide and protein level.

1148 The label-free and SILAC DIA datasets were extracted with the respective spectral libraries using 1149 Spectronaut v.15 (Biognosys AG). Default settings were used for the chromatogram extraction, except 1150 the machine learning option was set to "across experiment" and "cross run normalization" was 1151 excluded. The ion intensities at the fragment level were exported for further analysis in R. Raw MS 1152 data, the spectral libraries and the DIA data extractions generated with Spectronaut are uploaded in the 1153 PRIDE repository.

\section{Labeling quantification in affinity pulldowns}

1155 Analysis of protein labeling in KARMA assays with Brl1 bait was implemented in $\mathrm{R}$ 1156 ("Labeling_BRLIAP.R”). Initially, low quality fragment ions were excluded from further analysis based 1157 on the Spectronaut "F.ExcludedFromQuantification" flag. Additionally, only proteotypic y-type 1158 fragment ions with a single lysine residue that were found in both heavy and light channels were 1159 retained. The remaining fragment ion intensities were summed for each precursor in heavy and light 1160 channels as the respective precursor intensity. Unreliable precursor ions that were detected in fewer 1161 than two out of three biological replicates in any of the three post labeling time points $(30,60$ and 90 $1162 \mathrm{~min}$ ) were also excluded. The fractional labeling of the remaining precursor ions was then calculated as

$1163 \mathrm{H} /(\mathrm{H}+\mathrm{L})$, where $\mathrm{H}$ and $\mathrm{L}$ are the precursor intensities in heavy and light channels. The median protein 1164 labeling within each sample was computed as the median fractional labeling of all precursors. As an additional quality criterion, we also computed the root mean square error (RMSE) of the labeling values 1166 for every precursor from the respective protein median across all nine samples. For any protein, the 1167 precursors with the 50\% highest RMSEs were discarded, and the final protein labeling was computed 1168 as the median fractional labeling of the remaining high quality precursors. As a last filtration step, 1169 proteins with visually noisy labeling trajectories across the biological replicates and timepoints were 1170 excluded in a blinded manner. For the comparison of NUP labeling rates with Brl1 bait and ten NUP 
1172 S1C the median from three biological replicates was taken and labeling values were normalized to the 1173 bait labeling.

1174 Protein labeling in the Nup170 APs of Brl1-AID strains ("Labeling_NUP170AP_BRL1AID.R”) was 1175 analyzed the same way as for Brl1 APs, except that precursor ions found in at least one out of three 1176 replicates in all post labeling time points were also considered for quantification. The fractional labeling 1177 ratio between the auxin treated cells and the ethanol solvent control was calculated for each biological 1178 replicate and post labeling time point $(4 \mathrm{~h}, 4.5 \mathrm{~h}$ and $5 \mathrm{~h}$ ) and the average \pm SEM is plotted (Figure $4 \mathrm{C}$ 1179 D).

1180 For the lysate intermixing assays ("LysisIntermixingTest.R") the protein fractional labeling was 1181 quantified essentially as described above except that low intensity precursor ions $(<100)$ were filtered 1182 out and only proteins characterized by more than three precursor ions were considered (due to the low 1183 extent of intermixing, Brl1 bait is only characterized by two precursor ions that were found in both 1184 heavy and light). To get the intermixing extent, NUP fractional labeling was normalized to the mean 1185 fractional labeling of all co-purified proteins.

\section{Label-free quantification in affinity pulldowns}

1187 The exact specification of the quantitative analysis pipeline of protein abundances is given by the 1188 respective code in R (“Label-Free_BRL1AP.R”). In brief, NUP abundances in the affinity pulldown 1189 with Brl1 bait, low quality fragment ions were excluded based on Spectronaut 1190 "F.ExcludedFromQuantification" flag. For each proteotypic precursor ion all remaining fragment ions were summed and resulting intensities were median normalized across samples. Precursor ions that were not found in all three biological replicates were omitted. Protein intensities were calculated based on the average of the top3 most intense precursor ions, only considering NUPs and NTRs characterized

1194 by a minimum of three ions and also reproducibly found in the KARMA assay with Brl1 bait. The 1195 intensity of proteins in APs with NUP baits was essentially quantified the same, except that only 1196 precursor found in three replicates with all ten handles were considered for quantification. To assess the 1197 enrichment differences between the early and late tier baits for all 1523 co-purified proteins (Figure 1198 S1A), for each bait the median protein intensity of three biological replicates was taken. Then, the fold 1199 difference between the median of all baits from a respective assembly tier was calculated. To focus on non-NPC proteins NUPs and NTRs were excluded.

\section{Statistics and data visualization}

1202 No statistical method was used to estimate sample sizes. The statistical analysis and data exclusion criteria are discussed throughout the text. Statistical tests were carried out in R v. 4.1.2 (R Project),

1204 Excel (Microsoft) or Prism (GraphPad). The statistical test that was performed, sample size $\mathrm{n}$ and $\mathrm{P}$ 
values are indicated in the respective figure legends. Figure panels were generated using inkscape 1.1 and Adobe Illustrator v. 26.0.3 (Adobe).

\section{References}

Akey, Christopher W., Digvijay Singh, Christna Ouch, Ignacia Echeverria, Ilona Nudelman, Joseph M. Varberg, Zulin Yu, Fei Fang, Yi Shi, Junjie Wang, Daniel Salzberg, Kangkang Song, Chen Xu, James C. Gumbart, Sergey Suslov, Jay Unruh, Sue L. Jaspersen, Brian T. Chait, Andrej Sali, Javier Fernandez-Martinez, Steven J. Ludtke, Elizabeth Villa, and Michael P. Rout. 2022. "Comprehensive Structure and Functional Adaptations of the Yeast Nuclear Pore Complex." Cell 185(2):361-378.e25.

Alber, Frank, Svetlana Dokudovskaya, Liesbeth M. Veenhoff, Wenzhu Zhang, Julia Kipper, Damien Devos, Adisetyantari Suprapto, Orit Karni-Schmidt, Rosemary Williams, Brian T. Chait, Michael P. Rout, and Andrej Sali. 2007. "Determining the Architectures of Macromolecular Assemblies." Nature 450(7170):683-94.

Allegretti, Matteo, Christian E. Zimmerli, Vasileios Rantos, Florian Wilfling, Paolo Ronchi, Herman K. H. Fung, Chia-Wei Lee, Wim Hagen, Beata Turoňová, Kai Karius, Mandy Börmel, Xiaojie Zhang, Christoph W. Müller, Yannick Schwab, Julia Mahamid, Boris Pfander, Jan Kosinski, and Martin Beck. 2020. "In-Cell Architecture of the Nuclear Pore and Snapshots of Its Turnover." Nature 586(7831):796-800.

Bancaud, Aurélien, Sébastien Huet, Gwénaël Rabut, and Jan Ellenberg. 2010. "Fluorescence Perturbation Techniques to Study Mobility and Molecular Dynamics of Proteins in Live Cells: FRAP, Photoactivation, Photoconversion, and FLIP." Cold Spring Harbor Protocols 2010(12):pdb.top90.

Bley, Christopher J., Si Nie, George W. Mobbs, Stefan Petrovic, Anna T. Gres, Xiaoyu Liu, Somnath Mukherjee, Sho Harvey, Ferdinand M. Huber, Daniel H. Lin, Bonnie Brown, Aaron W. Tang, Emily J. Rundlet, Ana R. Correia, Shane Chen, Saroj G. Regmi, Mary Dasso, Alina Patke, Alexander F. Palazzo, Anthony A. Kossiakoff, and André Hoelz. 2021. "Architecture of the Cytoplasmic Face of the Nuclear Pore.” BioRxiv.

De Bruyn Kops, A. and C. Guthrie. 2001. "An Essential Nuclear Envelope Integral Membrane Protein, Brr6p, Required for Nuclear Transport.” EMBO Journal 20(15):4183-93.

Cibulka, Jakub, Fabio Bisaccia, Katarina Radisavljević, Ricardo M. Gudino Carrillo, and Alwin Köhler. 2022. "Assembly Principle of a Membrane-Anchored Nuclear Pore Basket Scaffold." Science Advances 8:44-47.

Dawson, T. Renee, Michelle D. Lazarus, Martin W. Hetzer, and Susan R. Wente. 2009. "ER MembraneBending Proteins Are Necessary for de Novo Nuclear Pore Formation." The Journal of Cell 
Biology 184(5):659-75.

Deng, Min and Mark Hochstrasser. 2006. "Spatially Regulated Ubiquitin Ligation by an ER/Nuclear Membrane Ligase." Nature 443(7113):827-31.

Doucet, Christine M. and Martin W. Hetzer. 2010. "Nuclear Pore Biogenesis into an Intact Nuclear Envelope." Chromosoma 119(5):469-77.

Doucet, Christine M., Jessica A. Talamas, and Martin W. Hetzer. 2010. "Cell Cycle-Dependent Differences in Nuclear Pore Complex Assembly in Metazoa." Cell 141(6):1030-41.

Drozdetskiy, Alexey, Christian Cole, James Procter, and Geoffrey J. Barton. 2015. "JPred4: A Protein Secondary Structure Prediction Server.” Nucleic Acids Research 43(W1):W389-94.

Eibauer, Matthias, Mauro Pellanda, Yagmur Turgay, Anna Dubrovsky, Annik Wild, and Ohad Medalia. 2015. "Structure and Gating of the Nuclear Pore Complex." Nature Communications 6(1):7532.

Escher, Claudia, Lukas Reiter, Brendan MacLean, Reto Ossola, Franz Herzog, John Chilton, Michael J. MacCoss, and Oliver Rinner. 2012. "Using IRT, a Normalized Retention Time for More Targeted Measurement of Peptides." Proteomics 12(8):1111-21.

Fernandez-Martinez, Javier and Michael P. Rout. 2021. "One Ring to Rule Them All? Structural and Functional Diversity in the Nuclear Pore Complex.” Trends in Biochemical Sciences 46(7):595607.

Floch, Aurélie G., David Tareste, Patrick F. J. Fuchs, Anne Chadrin, Ikrame Naciri, Thibaut Léger, Gabriel Schlenstedt, Benoit Palancade, and Valérie Doye. 2015. "Nuclear Pore Targeting of the Yeast Pom33 Nucleoporin Depends on Karyopherin and Lipid Binding." Journal of Cell Science 128(2):305-16.

Ford, Marijn G. J., Ian G. Mills, Brian J. Peter, Yvonne Vallis, Gerrit J. K. Praefcke, Philip R. Evans, and Harvey T. McMahon. 2002. "Curvature of Clathrin-Coated Pits Driven by Epsin." Nature 419(6905):361-66.

Friedrich, Förster, Medalia Ohad, Zauberman Nathan, Baumeister Wolfgang, and Fass Deborah. 2005. "Retrovirus Envelope Protein Complex Structure in Situ Studied by Cryo-Electron Tomography." Proceedings of the National Academy of Sciences 102(13):4729-34.

Gautier, Romain, Dominique Douguet, Bruno Antonny, and Guillaume Drin. 2008. "HELIQUEST: A Web Server to Screen Sequences with Specific Alpha-Helical Properties." Bioinformatics (Oxford, England) 24(18):2101-2.

Hagen, Wim J. H., William Wan, and John A. G. Briggs. 2017. "Implementation of a Cryo-Electron Tomography Tilt-Scheme Optimized for High Resolution Subtomogram Averaging." Journal of Structural Biology 197(2):191-98.

Hamed, Mohamed and Wolfram Antonin. 2021. "Dunking into the Lipid Bilayer: How Direct Membrane Binding of Nucleoporins Can Contribute to Nuclear Pore Complex Structure and Assembly." Cells 10(12):3601. 
Hartwell, Leland H., John J. Hopfield, Stanislas Leibler, and Andrew W. Murray. 1999. "From Molecular to Modular Cell Biology." Nature 402(6761):C47-52.

Hodge, Christine A., Vineet Choudhary, Michael J. Wolyniak, John J. Scarcelli, Roger Schneiter, and Charles N. Cole. 2010. "Integral Membrane Proteins Brr6 and Apq12 Link Assembly of the Nuclear Pore Complex to Lipid Homeostasis in the Endoplasmic Reticulum." Journal of Cell Science 123(1):141-51.

Huang, Gaoxingyu, Xiechao Zhan, Chao Zeng, Ke Liang, Xuechen Zhu, Yanyu Zhao, Pan Wang, Qifan Wang, Qiang Zhou, Qinghua Tao, Minhao Liu, Jianlin Lei, Chuangye Yan, and Yigong Shi. 2021. "Cryo-EM Structure of the Inner Ring from Xenopus Laevis Nuclear Pore Complex." BioRxiv. Huang, Gaoxingyu, Xiechao Zhan, Chao Zeng, Xuechen Zhu, Ke Liang, Yanyu Zhao, Pan Wang, Qifan Wang, Qiang Zhou, Qinghua Tao, Minhao Liu, Jianlin Lei, Chuangye Yan, and Yigong Shi. 2022. "Cryo-EM Structure of the Nuclear Ring from Xenopus Laevis Nuclear Pore Complex." Cell Research.

Jakub, Cibulka, Bisaccia Fabio, Radisavljević Katarina, Gudino Carrillo Ricardo M., and Köhler Alwin. 2022. "Assembly Principle of a Membrane-Anchored Nuclear Pore Basket Scaffold." Science Advances 8(6):eabl6863.

Jumper, John, Richard Evans, Alexander Pritzel, Tim Green, Michael Figurnov, Olaf Ronneberger, Kathryn Tunyasuvunakool, Russ Bates, Augustin Žídek, Anna Potapenko, Alex Bridgland, Clemens Meyer, Simon A. A. Kohl, Andrew J. Ballard, Andrew Cowie, Bernardino RomeraParedes, Stanislav Nikolov, Rishub Jain, Jonas Adler, Trevor Back, Stig Petersen, David Reiman, Ellen Clancy, Michal Zielinski, Martin Steinegger, Michalina Pacholska, Tamas Berghammer, Sebastian Bodenstein, David Silver, Oriol Vinyals, Andrew W. Senior, Koray Kavukcuoglu, Pushmeet Kohli, and Demis Hassabis. 2021. "Highly Accurate Protein Structure Prediction with AlphaFold." Nature 596(7873):583-89.

Kressler, Dieter, Ed Hurt, and Jochen Bassler. 2010. "Driving Ribosome Assembly." Biochimica et Biophysica Acta 1803(6):673-83.

Laudermilch, Ethan, Pei-Ling Tsai, Morven Graham, Elizabeth Turner, Chenguang Zhao, and Christian Schlieker. 2016. "Dissecting Torsin/Cofactor Function at the Nuclear Envelope: A Genetic Study." Molecular Biology of the Cell 27(25):3964-71.

Li, Zongqiang, Shuaijiabin Chen, Liang Zhao, Guoqiang Huang, Xiong Pi, Shan Sun, Peiyi Wang, and Sen-Fang Sui. 2021. "Near Atomic Structure of the Inner Ring of the Saccharomyces Cerevisiae Nuclear Pore Complex." BioRxiv.

Lin, Daniel H. and André Hoelz. 2019. "The Structure of the Nuclear Pore Complex (An Update)." Annual Review of Biochemistry 88:725-83.

Liu, Gaowen, Mei Yun Jacy Yong, Marina Yurieva, Kandhadayar Gopalan Srinivasan, Jaron Liu, John Soon Yew Lim, Michael Poidinger, Graham Daniel Wright, Francesca Zolezzi, Hyungwon Choi, 
Norman Pavelka, and Giulia Rancati. 2015. "Gene Essentiality Is a Quantitative Property Linked to Cellular Evolvability." Cell 163(6):1388-99.

Lone, Museer A., Aaron E. Atkinson, Christine A. Hodge, Stéphanie Cottier, Fernando MartínezMontañés, Shelley Maithel, Laurent Mène-Saffrané, Charles N. Cole, and Roger Schneiter. 2015. "Yeast Integral Membrane Proteins Apq12, Brl1, and Brr6 Form a Complex Important for Regulation of Membrane Homeostasis and Nuclear Pore Complex Biogenesis." Eukaryotic Cell 14(12):1217-27.

Makio, Tadashi, Leslie H. Stanton, Cheng-Chao Lin, David S. Goldfarb, Karsten Weis, and Richard W. Wozniak. 2009. "The Nucleoporins Nup170p and Nup157p Are Essential for Nuclear Pore Complex Assembly.” Journal of Cell Biology 185(3):459-73.

Marelli, Marcello, C. Patrick Lusk, Honey Chan, John D. Aitchison, and Richard W. Wozniak. 2001. "A Link between the Synthesis of Nucleoporins and the Biogenesis of the Nuclear Envelope." Journal of Cell Biology 153(4):709-24.

Mastronarde, David N. 2003. "SerialEM: A Program for Automated Tilt Series Acquisition on Tecnai Microscopes Using Prediction of Specimen Position." Microscopy and Microanalysis 9(S02):1182-83.

Mastronarde, David N. and Susannah R. Held. 2017. "Automated Tilt Series Alignment and Tomographic Reconstruction in IMOD.” Journal of Structural Biology 197(2):102-13.

Mészáros, Noémi, Jakub Cibulka, Maria Jose Mendiburo, Anete Romanauska, Maren Schneider, and Alwin Köhler. 2015. "Nuclear Pore Basket Proteins Are Tethered to the Nuclear Envelope and Can Regulate Membrane Curvature.” Developmental Cell 33(3):285-98.

Morin, Andrew, Ben Eisenbraun, Jason Key, Paul C. Sanschagrin, Michael A. Timony, Michelle Ottaviano, and Piotr Sliz. 2013. "Cutting Edge: Collaboration Gets the Most out of Software." ELife 2:e1456.

Mosalaganti, Shyamal, Agnieszka Obarska-Kosinska, Marc Siggel, Beata Turonova, Christian E. Zimmerli, Katarzyna Buczak, Florian H. Schmidt, Erica Margiotta, Marie-Therese Mackmull, Wim Hagen, Gerhard Hummer, Martin Beck, and Jan Kosinski. 2021. "Artificial Intelligence Reveals Nuclear Pore Complexity.” BioRxiv 2021.10.26.465776.

Nickell, Stephan, Friedrich Förster, Alexandros Linaroudis, William Del Net, Florian Beck, Reiner Hegerl, Wolfgang Baumeister, and Jürgen M. Plitzko. 2005. "TOM Software Toolbox: Acquisition and Analysis for Electron Tomography." Journal of Structural Biology 149(3):22734.

Nishimura, Kohei, Tatsuo Fukagawa, Haruhiko Takisawa, Tatsuo Kakimoto, and Masato Kanemaki. 2009. "An Auxin-Based Degron System for the Rapid Depletion of Proteins in Nonplant Cells." Nature Methods 6(12):917-22.

Onischenko, Evgeny, Elad Noor, Jonas S. Fischer, Ludovic Gillet, Matthias Wojtynek, Pascal 
Vallotton, and Karsten Weis. 2020. "Maturation Kinetics of a Multiprotein Complex Revealed by Metabolic Labeling." Cell 183(7):1785-1800.e26.

Onischenko, Evgeny, Leslie H. Stanton, Alexis S. Madrid, Thomas Kieselbach, and Karsten Weis. 2009. "Role of the Ndc1 Interaction Network in Yeast Nuclear Pore Complex Assembly and Maintenance." The Journal of Cell Biology 185(3):475-91.

Onischenko, Evgeny, Jeffrey H. Tang, Kasper R. Andersen, Kevin E. Knockenhauer, Pascal Vallotton, Carina P. Derrer, Annemarie Kralt, Christopher F. Mugler, Leon Y. Chan, Thomas U. Schwartz, and Karsten Weis. 2017. "Natively Unfolded FG Repeats Stabilize the Structure of the Nuclear Pore Complex." Cell 171(4):904-917.e19.

Otsuka, Shotaro, Khanh Huy Bui, Martin Schorb, M. Julius Hossain, Antonio Z. Politi, Birgit Koch, Mikhail Eltsov, Martin Beck, and Jan Ellenberg. 2016. "Nuclear Pore Assembly Proceeds by an Inside-out Extrusion of the Nuclear Envelope." ELife 5(September2016):1-23.

Otsuka, Shotaro and Jan Ellenberg. 2018. "Mechanisms of Nuclear Pore Complex Assembly - Two Different Ways of Building One Molecular Machine." FEBS Letters 592(4):475-88.

Peeters, Bas W. A., Alexandra C. A. Piët, and Maarten Fornerod. 2022. "Generating Membrane Curvature at the Nuclear Pore: A Lipid Point of View." Cells 11(3).

Peterson, Amelia C., Jason D. Russell, Derek J. Bailey, Michael S. Westphall, and Joshua J. Coon. 2012. "Parallel Reaction Monitoring for High Resolution and High Mass Accuracy Quantitative, Targeted Proteomics.” Molecular \& Cellular Proteomics : MCP 11(11):1475-88.

Petrovic, Stefan, Dipanjan Samanta, Thibaud Perriches, Christopher J. Bley, Karsten Thierbach, Bonnie Brown, Si Nie, George W. Mobbs, Taylor A. Stevens, Xiaoyu Liu, and André Hoelz. 2021. "Architecture of the Linker-Scaffold in the Nuclear Pore." BioRxiv.

Pettersen, Eric F., Thomas D. Goddard, Conrad C. Huang, Gregory S. Couch, Daniel M. Greenblatt, Elaine C. Meng, and Thomas E. Ferrin. 2004. "UCSF Chimera--a Visualization System for Exploratory Research and Analysis.” Journal of Computational Chemistry 25(13):1605-12.

Popken, Petra, Ali Ghavami, Patrick R. Onck, Bert Poolman, and Liesbeth M. Veenhoff. 2015. "SizeDependent Leak of Soluble and Membrane Proteins through the Yeast Nuclear Pore Complex." Molecular Biology of the Cell 26(7):1386-94.

Rajoo, Sasikumar, Pascal Vallotton, Evgeny Onischenko, and Karsten Weis. 2018. "Stoichiometry and Compositional Plasticity of the Yeast Nuclear Pore Complex Revealed by Quantitative Fluorescence Microscopy." Proceedings of the National Academy of Sciences 115(17):E3969-77. Rampello, Anthony J., Ethan Laudermilch, Nidhi Vishnoi, Sarah M. Prophet, Lin Shao, Chenguang Zhao, C. Patrick Lusk, and Christian Schlieker. 2020. "Torsin ATPase Deficiency Leads to Defects in Nuclear Pore Biogenesis and Sequestration of MLF2." The Journal of Cell Biology 219(6).

Romanauska, Anete and Alwin Köhler. 2018. "The Inner Nuclear Membrane Is a Metabolically Active 
Territory That Generates Nuclear Lipid Droplets." Cell 174(3):700-715.e18.

Rothballer, Andrea and Ulrike Kutay. 2013. "Poring over Pores: Nuclear Pore Complex Insertion into the Nuclear Envelope." Trends in Biochemical Sciences 38(6):292-301.

Ryan, Kathryn J., J. Michael McCaffery, and Susan R. Wente. 2003. "The Ran GTPase Cycle Is Required for Yeast Nuclear Pore Complex Assembly.” The Journal of Cell Biology 160(7):104153.

Saitoh, Yoh Hei, Kaoru Ogawa, and Takeharu Nishimoto. 2005. "Brl1p - A Novel Nuclear Envelope Protein Required for Nuclear Transport." Traffic 6(6):502-17.

Scarcelli, John J., Christine A. Hodge, and Charles N. Cole. 2007. "The Yeast Integral Membrane Protein Apq12 Potentially Links Membrane Dynamics to Assembly of Nuclear Pore Complexes." Journal of Cell Biology 178(5):799-812.

Schindelin, Johannes, Ignacio Arganda-Carreras, Erwin Frise, Verena Kaynig, Mark Longair, Tobias Pietzsch, Stephan Preibisch, Curtis Rueden, Stephan Saalfeld, Benjamin Schmid, Jean-Yves Tinevez, Daniel James White, Volker Hartenstein, Kevin Eliceiri, Pavel Tomancak, and Albert Cardona. 2012. "Fiji: An Open-Source Platform for Biological-Image Analysis." Nature Methods 9(7):676-82.

Schooley, Allana, Benjamin Vollmer, and Wolfram Antonin. 2012. "Building a Nuclear Envelope at the End of Mitosis: Coordinating Membrane Reorganization, Nuclear Pore Complex Assembly, and Chromatin de-Condensation." Chromosoma 121(6):539-54.

Schuller, Anthony P., Matthias Wojtynek, David Mankus, Meltem Tatli, Rafael Kronenberg-Tenga, Saroj G. Regmi, Phat V Dip, Abigail K. R. Lytton-Jean, Edward J. Brignole, Mary Dasso, Karsten Weis, Ohad Medalia, and Thomas U. Schwartz. 2021. "The Cellular Environment Shapes the Nuclear Pore Complex Architecture.” Nature 598(7882):667-71.

Strunk, Bethany S. and Katrin Karbstein. 2009. "Powering through Ribosome Assembly." RNA (New York, N.Y.) 15(12):2083-2104.

Tackett, Alan J., Jeffrey A. DeGrasse, Matthew D. Sekedat, Marlene Oeffinger, Michael P. Rout, and Brian T. Chait. 2005. "I-DIRT, a General Method for Distinguishing between Specific and Nonspecific Protein Interactions." Journal of Proteome Research 4(5):1752-56.

Tai, Linhua, Yun Zhu, He Ren, Xiaojun Huang, Chuanmao Zhang, and Fei Sun. 2022. "8 Å Structure of the Outer Rings of the Xenopus Laevis Nuclear Pore Complex Obtained by Cryo-EM and AI.” Protein \& Cell.

Thaller, David J. and C. Patrick Lusk. 2018. "Fantastic Nuclear Envelope Herniations and Where to Find Them." Biochemical Society Transactions 46(4):877-89.

Thaller, David J., Danqing Tong, Christopher J. Marklew, Nicholas R. Ader, Philip J. Mannino, Sapan Borah, Megan C. King, Barbara Ciani, and C. Patrick Lusk. 2021. "Direct Binding of ESCRT Protein Chm7 to Phosphatidic Acid-Rich Membranes at Nuclear Envelope Herniations.” The 
Journal of Cell Biology 220(3).

Upla, Paula, Seung Joong Kim, Parthasarathy Sampathkumar, Kaushik Dutta, Sean M. Cahill, Ilan E. Chemmama, Rosemary Williams, Jeffrey B. Bonanno, William J. Rice, David L. Stokes, David Cowburn, Steven C. Almo, Andrej Sali, Michael P. Rout, and Javier Fernandez-Martinez. 2017. "Molecular Architecture of the Major Membrane Ring Component of the Nuclear Pore Complex." Structure (London, England: 1993) 25(3):434-45.

Vallotton, Pascal, Sasikumar Rajoo, Matthias Wojtynek, Evgeny Onischenko, Annemarie Kralt, Carina Patrizia Derrer, and Karsten Weis. 2019. "Mapping the Native Organization of the Yeast Nuclear Pore Complex Using Nuclear Radial Intensity Measurements." Proceedings of the National Academy of Sciences 116(29):14606 LP - 14613.

Verzijlbergen, Kitty F., Victoria Menendez-Benito, Tibor van Welsem, Sjoerd J. van Deventer, Derek L. Lindstrom, Huib Ovaa, Jacques Neefjes, Daniel E. Gottschling, and Fred van Leeuwen. 2010. "Recombination-Induced Tag Exchange to Track Old and New Proteins." Proceedings of the National Academy of Sciences 107(1):64 LP - 68.

Voeltz, Gia K., William A. Prinz, Yoko Shibata, Julia M. Rist, and Tom A. Rapoport. 2006. "A Class of Membrane Proteins Shaping the Tubular Endoplasmic Reticulum." Cell 124(3):573-86.

Vollmer, Benjamin, Michael Lorenz, Daniel Moreno-Andrés, Mona Bodenhöfer, Paola De Magistris, Susanne Adina Astrinidis, Allana Schooley, Matthias Flötenmeyer, Sebastian Leptihn, and Wolfram Antonin. 2015. "Nup153 Recruits the Nup107-160 Complex to the Inner Nuclear Membrane for Interphasic Nuclear Pore Complex Assembly." Developmental Cell 33(6):717-28. Wagner, Felix R., Reika Watanabe, Ruud Schampers, Digvijay Singh, Hans Persoon, Miroslava Schaffer, Peter Fruhstorfer, Jürgen Plitzko, and Elizabeth Villa. 2020. "Preparing Samples from Whole Cells Using Focused-Ion-Beam Milling for Cryo-Electron Tomography." Nature Protocols 15(6):2041-70.

Wang, Ning, Lindsay D. Clark, Yuan Gao, Michael M. Kozlov, Tom Shemesh, and Tom A. Rapoport. 2021. "Mechanism of Membrane-Curvature Generation by ER-Tubule Shaping Proteins." Nature Communications 12(1):568.

Wang, Songyu, Hanna Tukachinsky, Fabian B. Romano, and Tom A. Rapoport. 2016. "Cooperation of the ER-Shaping Proteins Atlastin, Lunapark, and Reticulons to Generate a Tubular Membrane Network" edited by M. Kozlov. ELife 5:e18605.

Waterhouse, Andrew M., James B. Procter, David M. A. Martin, Michèle Clamp, and Geoffrey J. Barton. 2009. "Jalview Version 2-a Multiple Sequence Alignment Editor and Analysis Workbench." Bioinformatics 25(9):1189-91.

Wente, Susan R. and Michael P. Rout. 2010. “The Nuclear Pore Complex and Nuclear Transport.” Cold Spring Harbor Perspectives in Biology 2(10):a000562.

Winey, M., D. Yarar, T. H. Giddings Jr, and D. N. Mastronarde. 1997. "Nuclear Pore Complex Number 
and Distribution throughout the Saccharomyces Cerevisiae Cell Cycle by Three-Dimensional Reconstruction from Electron Micrographs of Nuclear Envelopes." Molecular Biology of the Cell 8(11):2119-32.

Wright, R., M. Basson, L. D’Ari, and J. Rine. 1988. "Increased Amounts of HMG-CoA Reductase Induce 'Karmellae': A Proliferation of Stacked Membrane Pairs Surrounding the Yeast Nucleus." The Journal of Cell Biology 107(1):101-14.

Zhang, Wanlu, Azqa Khan, Jlenia Vitale, Annett Neuner, Kerstin Rink, Christian Lüchtenborg, Britta Brügger, Thomas H. Söllner, and Elmar Schiebel. 2021. "A Short Perinuclear Amphipathic $\alpha$ Helix in Apq12 Promotes Nuclear Pore Complex Biogenesis.” Open Biology 11(11).

Zhang, Wanlu, Annett Neuner, Diana Rüthnick, Timo Sachsenheimer, Christian Lüchtenborg, Britta Brügger, and Elmar Schiebel. 2018. "Brr6 and Brl1 Locate to Nuclear Pore Complex Assembly Sites to Promote Their Biogenesis." Journal of Cell Biology 217(3):877-94.

Zhu, Xuechen, Gaoxingyu Huang, Chao Zeng, Xiechao Zhan, Ke Liang, Yanyu Zhao, Pan Wang, Qifan Wang, Qiang Zhou, Qinghua Tao, Minhao Liu, Jianlin Lei, Chuangye Yan, and Yigong Shi. 2022. "Near-Atomic Structure of the Cytoplasmic Ring of the Xenopus Laevis Nuclear Pore Complex." BioRxiv.

Zimmerli, Christian, Allegretti Matteo, Rantos Vasileios, Goetz Sara K., Obarska-Kosinska Agnieszka, Zagoriy Ievgeniia, Halavatyi Aliaksandr, Hummer Gerhard, Mahamid Julia, Kosinski Jan, and Beck Martin. 2022. “Nuclear Pores Dilate and Constrict in Cellulo.” Science 374(6573):eabd9776. 